Aus der Abteilung Allgemeinmedizin

(Prof. Dr. med. M. M. Kochen, MPH, FRCGP)

im Zentrum Innere Medizin

der Medizinischen Fakultät der Universität Göttingen

\title{
Diagnostik und Therapie von Atemwegsinfekten in der Allgemeinarztpraxis
}

Erhebung an 273 Patienten bei 30 Fachärzten für Allgemeinmedizin

\author{
Inaugural - Dissertation \\ zur Erlangung des Doktorgrades \\ der Medizinischen Fakultät \\ der Georg-August-Universität zu Göttingen
}

vorgelegt von

Susanne Fischer, geb. Hecker

aus

Brilon

Göttıngen 2003 
D e k a n: $\quad$ Prof. Dr. med. M. Droese

I. Berichterstatter: Prof. Dr. med. M. M. Kochen, MPH, FRCGP

II. Berichterstatter/in: Prof. Dr. med. U. Hüttemann

III. Berichterstatter/in:

Tag der mündlichen Prüfung: 03. Februar 2004 


\section{Inhaltsverzeichnis}

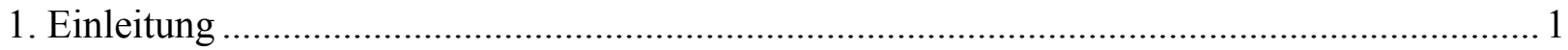

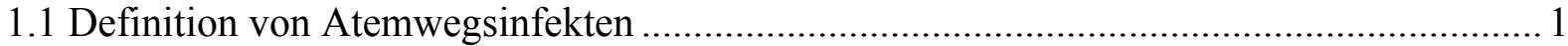

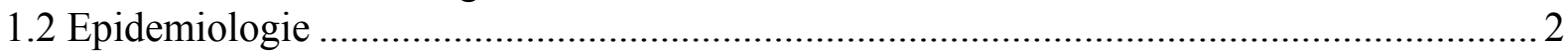

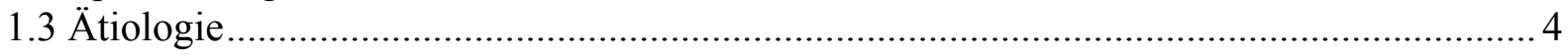

1.4 Evidenzbasierte Therapie ......................................................................................... 5

1.5 Arzneiverordnungen bei akuten Atemwegsinfekten in Deutschland .................................. 8

1.6 Mögliche Einflussfaktoren auf das Verordnungsverhalten der Ärzte................................. 9

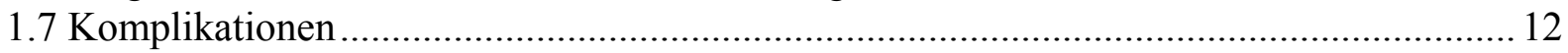

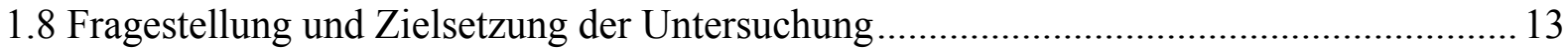

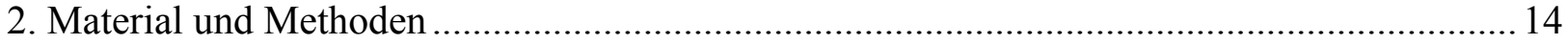

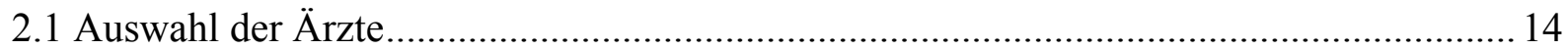

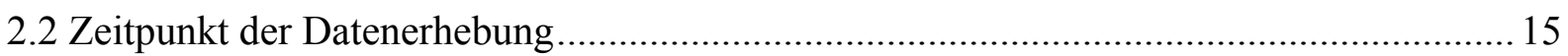

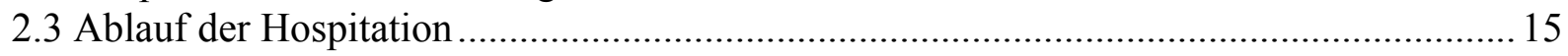

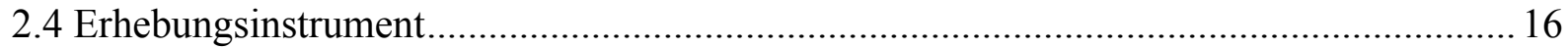

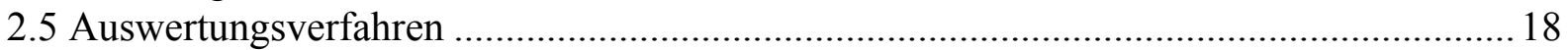

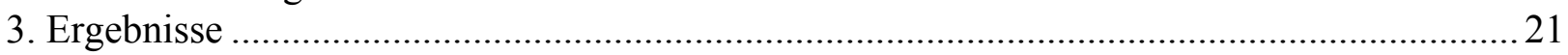

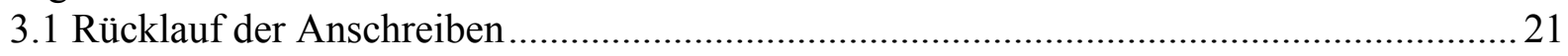

3.2 Beschreibung der Stichprobe und des Patientenkollektivs ............................................... 21

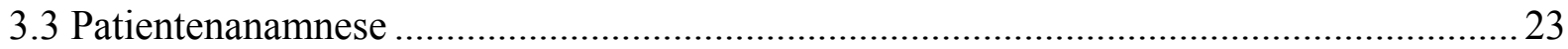

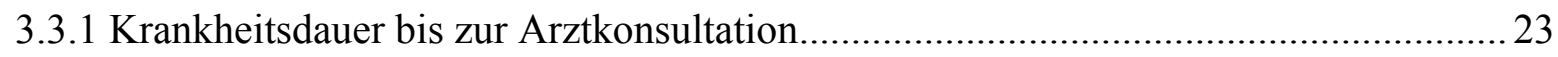

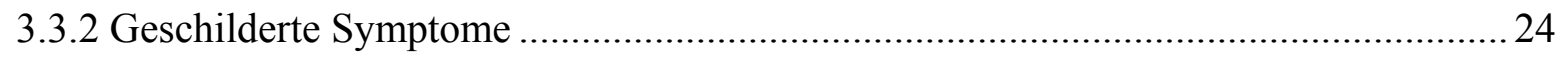

3.3.2.1 Patientenanamnese zum Symptom Fieber........................................................25

3.3.2.2 Patientenanamnese zum Symptom Husten ........................................................ 25

3.3.3 Selbstmedikation und nichtmedikamentöse Therapie.................................................2. 26

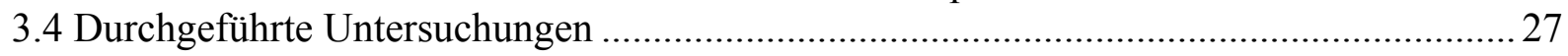

3.4.1 Körperliche Untersuchungen und Untersuchungsbefunde .......................................2.

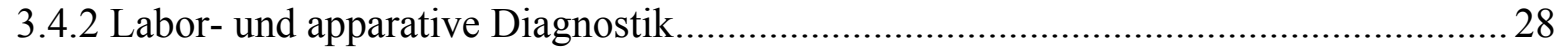

3.4.3 Überweisung zu Spezialisten oder ins Krankenhaus ...............................................22

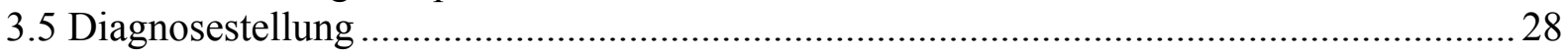

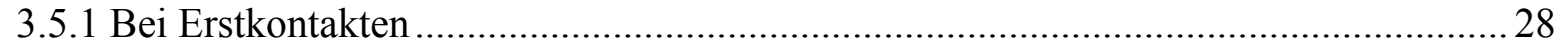

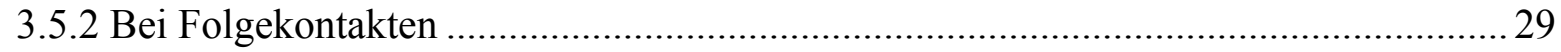

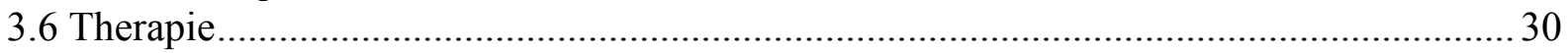

3.6.1 Anzahl der verordneten Medikamente pro Patient ……………….............................. 30

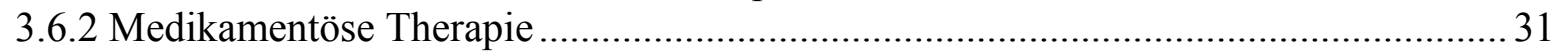

3.6.3 Verordnung von pflanzlichen Arzneien und Homöopathika ....................................... 32

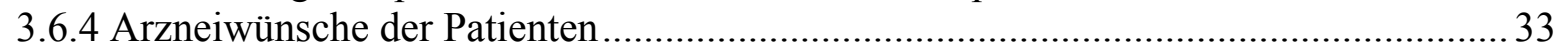

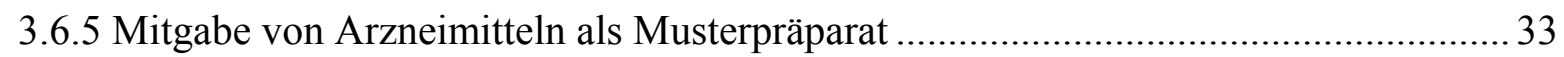

3.6.6 Empfehlungen zum Selbstkauf von Medikamenten ................................................. 34

3.6.7 Ausstellung einer Arbeitsunfähigkeitsbescheinigung (AU) .......................................34

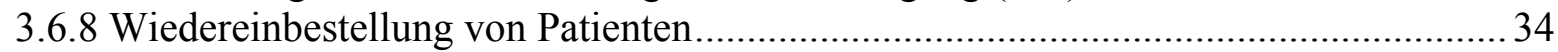

3.6.9 Empfehlungen zur Selbstmedikation und nichtmedikamentösen Therapie .................. 35

3.6.9.1 Zusätzliche Empfehlungen im Bezug auf die Verordnung von Acetylcystein ....36

3.6.9.2 Raucheranamnese und Erteilung eines Rauchverbotes...........................................36

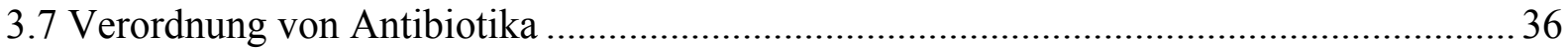

3.7.1 Unterscheidung der Antibiotika nach ihrer chemischen Substanz................................36 


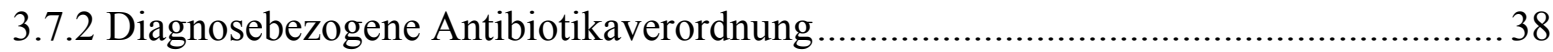

3.7.3 Einfluss von Symptomen und Untersuchungsbefunden auf die Antibiotikaverordnung

3.7.4 Praxischarakteristika als Einflussfaktoren auf die Antibiotikaverordnung ................. 41

3.8 Verordnung von Husten- und Erkältungspräparaten......................................................4 42

3.8.1 Diagnosebezogene Verordnung von Husten- und Erkältungspräparaten ..................... 42

3.8.2 Einfluss von Symptomen und Untersuchungsbefunden auf die Verordnung von Husten- und Erkältungspräparaten............................................................................... 43

3.8.3 Praxischarakteristika als Einflussfaktoren auf die Verordnung von Husten- und

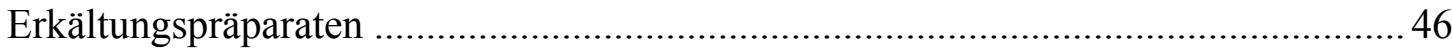

3.8.4 Verordnung von Antitussiva und mögliche Einflussfaktoren auf die Verordnung ..... 46

3.9 Diagnostik und medikamentöse Therapie der 4 häufigsten Krankheitsbilder .....................4 47

3.9.1 Diagnostik und medikamentöse Therapie des oberen Atemwegsinfektes .................... 47

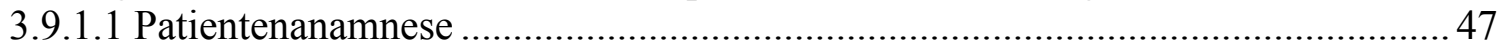

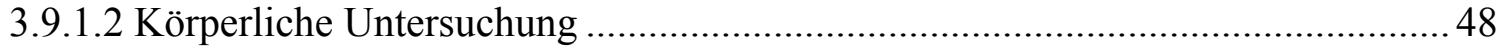

3.9.1.3 Abhängigkeit der Diagnosestellung von Symptomen und Untersuchungsbefunden

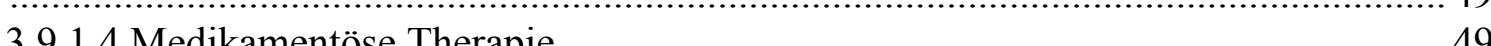

3.9.2 Diagnostik und medikamentöse Therapie der akuten Bronchitis ................................51

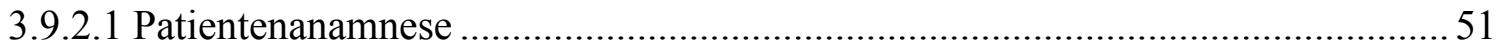

3.9.2.2 Körperliche Untersuchung .............................................................................. 51

3.9.2.3 Abhängigkeit der Diagnosestellung von Symptomen und Untersuchungsbefunden

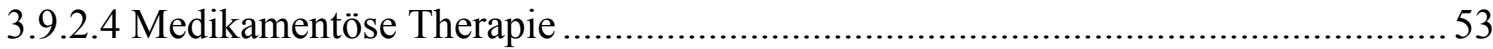

3.9.3 Diagnostik und medikamentöse Therapie der akuten Tonsillitis.................................. 54

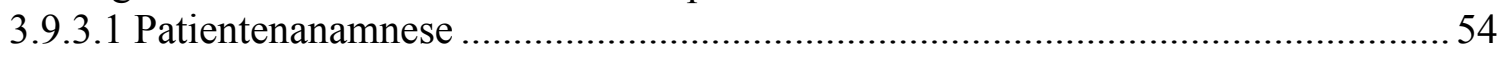

3.9.3.2 Körperliche Untersuchung ……………………............................................... 55

3.9.3.3 Abhängigkeit der Diagnosestellung von Symptomen und Untersuchungsbefunden ...................55

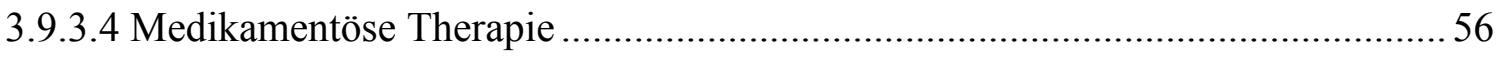

3.9.4 Diagnostik und medikamentöse Therapie der akuten Sinusitis ...................................5 57

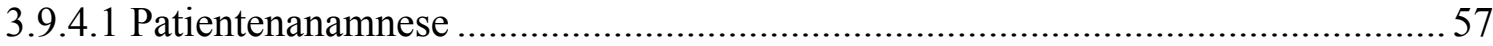

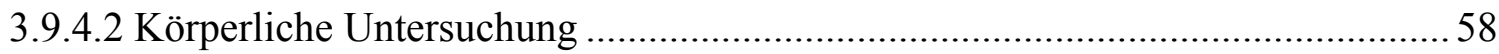

3.9.4.3 Abhängigkeit der Diagnosestellung von Symptomen und Untersuchungsbefunden

.

3.9.4.4 Medikamentöse Therapie …………………...............................................5 59

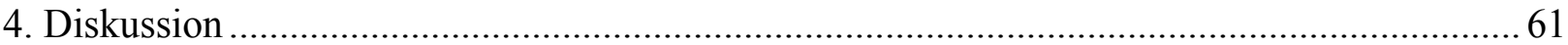

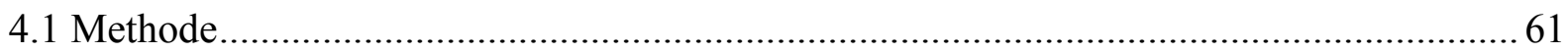

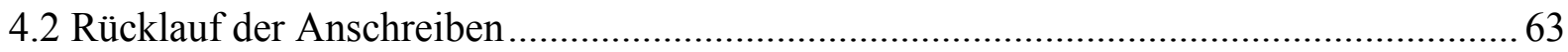

4.3 Beschreibung der Stichprobe und des Patientenkollektivs .................................................6 63

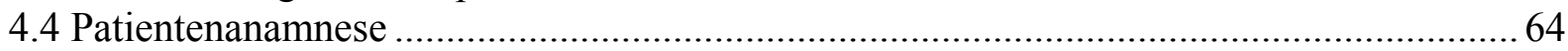

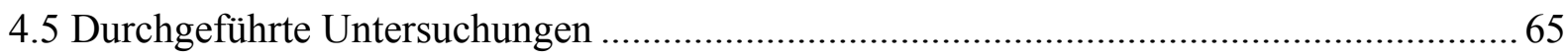

4.5.1 Körperliche Untersuchung und Untersuchungsbefund ..............................................6 65

4.5.2 Labor- und apparative Diagnostik............................................................................6 66

4.5.3 Überweisung zu Spezialisten oder ins Krankenhaus ......................................................67 67

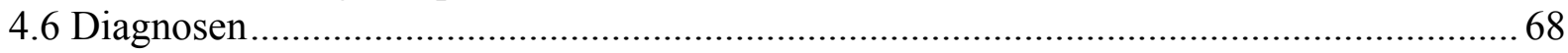

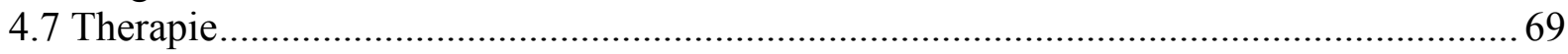


4.7.1 Art und Häufigkeit der Medikamentenverschreibung ..................................................69

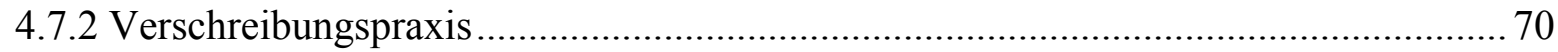

4.7.3 Ausstellung einer Arbeitsunfähigkeitsbescheinigung (AU), Wiedereinbestellung und

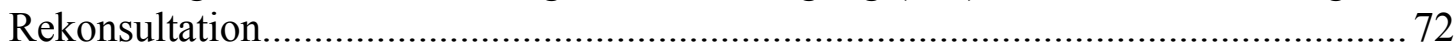

4.7.4 Selbstmedikation und vom Patienten durchgeführte nichtmedikamentöse Therapie .. 74

4.8 Verordnung von Antibiotika bei Atemwegsinfekten .......................................................

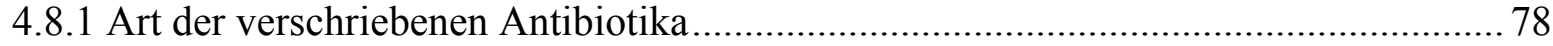

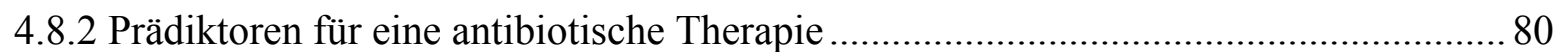

4.8.3 Bewältigungsstrategien zur Vermeidung unnötiger Antibiotikaverordnungen ............ 82

4.9 Verordnung von Husten- und Erkältungspräparaten......................................................... 84

4.10 Diagnostik und medikamentöse Therapie der 4 häufigsten Krankheitsbilder ...................8 87

4.10.1 Diagnostik und medikamentöse Therapie des oberen Atemwegsinfektes ..................8 87

4.10.2 Diagnostik und medikamentöse Therapie der akuten Bronchitis .............................. 88

4.10.3 Diagnostik und medikamentöse Therapie der akuten Tonsillitis............................... 92

4.10.4 Diagnostik und medikamentöse Therapie der akuten Sinusitis ................................. 97

4.11 Abhängigkeit der Diagnosestellung von geschilderten Symptomen und erhobenen

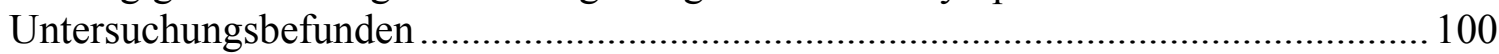

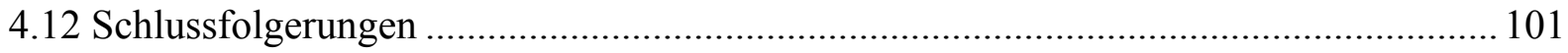

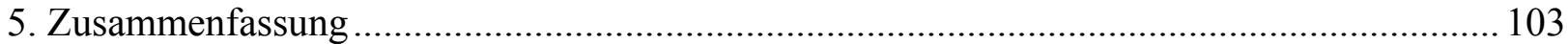

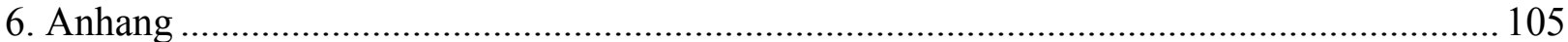

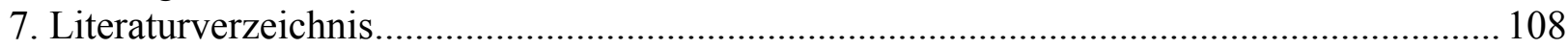

\section{Abbildungsverzeichnis:}

Abbildung 1: Altersverteilung bei Erst- und Folgekontakten ...................................................23

Abbildung 2: Medikamentöse Therapie des oberen Atemwegsinfektes.......................................50

Abbildung 3: Medikamentöse Therapie der akuten Bronchitis ....................................................53

Abbildung 4: Medikamentöse Therapie der akuten Tonsillitis ......................................................56

Abbildung 5: Medikamentöse Therapie der akuten Sinusitis ........................................................59 


\section{Tabellenverzeichnis:}

Tabelle 1: Virale Erreger und entsprechende Erkrankung/Symptomatik nach Adam et al. (1991)4

Tabelle 2: Differenzierung der Stichprobe und des Patientenkollektivs nach den Orten der

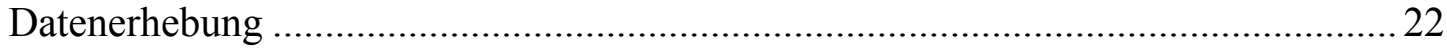

Tabelle 3: Art und Häufigkeit der geschilderten Symptome ......................................................2 24

Tabelle 4: Weiterführende Angaben zum Symptom Husten ………......................................2.25

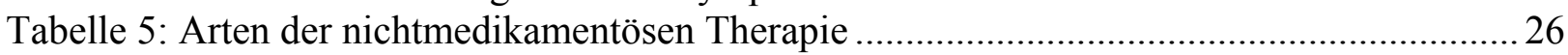

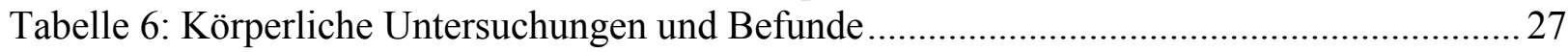

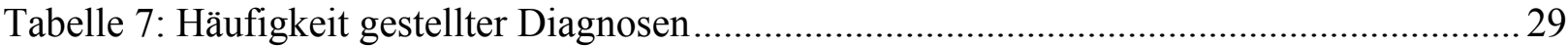

Tabelle 8: Häufigkeit gestellter Diagnosen bei Folgekontakten .................................................29

Tabelle 9: Anzahl der verordneten Medikamente pro Patient ...................................................... 31

Tabelle 10: Medikamentöse Therapie ......................................................................................... 31

Tabelle 11: Anzahl der Verordnungen innerhalb der pharmakologischen Hauptgruppen der

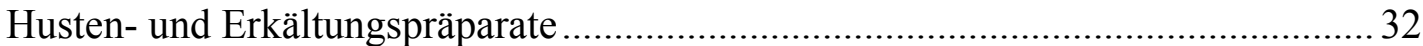

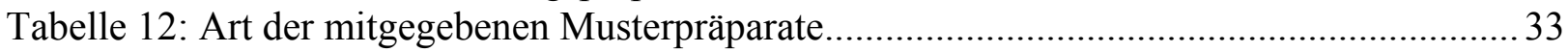

Tabelle 13: Empfehlungen zur nichtmedikamentösen Therapie....................................................35

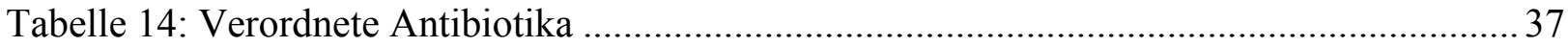

Tabelle 15: Diagnosebezogene Verordnung von Antibiotika......................................................38

Tabelle 16: Einfluss der geschilderten Symptome auf die Verordnung von Antibiotika .............39

Tabelle 17: Abhängigkeit der Antibiotikatherapie von Symptomen und Untersuchungsbefunden mittels multipler logistischer Regressionsanalyse ...................................................4 41

Tabelle 18: Diagnosebezogene Verordnung von Husten- und Erkältungspräparaten ................... 43

Tabelle 21: Körperliche Untersuchung beim oberen Atemwegsinfekt.........................................4 48

Tabelle 22: Abhängigkeit der Diagnosestellung oberer Atemwegsinfekt von Symptomen und

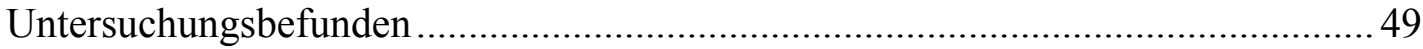

Tabelle 23: Antibiotikaverordnungen beim oberen Atemwegsinfekt ..........................................50

Tabelle 24: Körperliche Untersuchung bei akuter Bronchitis......................................................52

Tabelle 25: Abhängigkeit der Diagnosestellung akute Bronchitis von Symptomen und

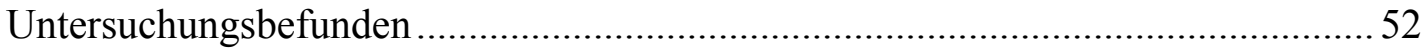

Tabelle 26: Antibiotikaverordnungen bei akuter Bronchitis.....................................................5 54

Tabelle 27: Körperliche Untersuchung bei akuter Tonsillitis ...................................................5

Tabelle 28: Abhängigkeit der Diagnosestellung akute Tonsillitis von Symptomen und

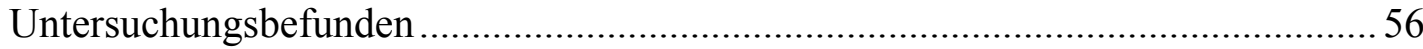

Tabelle 29: Antibiotikaverordnungen bei akuter Tonsillitis .......................................................57

Tabelle 30: Körperliche Untersuchung bei akuter Sinusitis ......................................................58

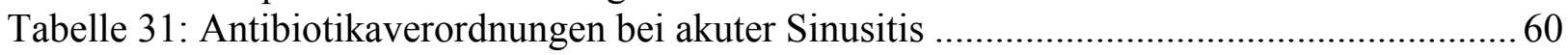




\title{
1. Einleitung
}

\subsection{Definition von Atemwegsinfekten}

\author{
„Ein Schnupfen hockt auf der Terrasse, \\ auf dass er sich ein Opfer fasse, \\ und stürzt alsbald mit großem Grimm \\ auf einen Menschen namens Schrimm. \\ Paul Schrimm erwidert prompt 'pitschü!' \\ und hat ihn drauf bis Montag früh.“"
}

(Morgenstern 2001, S.162)

Christian Morgenstern bezieht sich in seinem Gedicht „Der Schnupfen” auf eine häufige Erkrankung, mit der Menschen Jahr für Jahr konfrontiert werden. Die Definition akuter Atemwegsinfekte ist jedoch uneinheitlich. Glasziou und Del Mar (2002) definieren Infektionen der oberen Atemwege als Entzündungen im Bereich zwischen Nase und unterem Respirationstrakt mit Ausnahme des Alveolarraumes. Diese führen neben einem allgemeinen Krankheitsgefühl zu überlappenden Krankheitsbildern: Halsschmerzen (Pharyngitis), Schnupfen (Rhinitis), Nasennebenhöhlenentzündung (Sinusitis) und Husten (Bronchitis).

Dies deckt sich mit der Hypothese von Hueston et al. (2000 a), dass Sinusitis, oberer Atemwegsinfekt und Bronchitis Varianten der gleichen klinischen Erkrankung sind und als eine einzige klinische Einheit mit unterschiedlichen anatomischen Manifestationen gesehen werden sollten. Die Autoren begründen dies anhand von retrospektiven Daten von 544 Patienten mit diesen Diagnosen. Bei den einzelnen Krankheitsbildern konnten sich überschneidende Symptome dargestellt werden.

Im Gegensatz dazu steht die gegenwärtige Verpflichtung der Ärzte in Deutschland nach der „Internationalen statistischen Klassifikation der Krankheiten und verwandter Gesundheitsprobleme“, kurz ICD 10, zu kodieren, in der die verschiedenen Varianten von Atemwegsinfekten als getrennte Krankheiten klassifiziert werden (Deutsches Institut für 
medizinische Dokumentation und Information 1999). Bezogen auf die akuten Erkrankungen des Atmungssystems werden folgende Gruppen unterschieden:

- akute Infektionen der oberen Atemwege: akute Rhinopharyngitis (Erkältungsschnupfen), akute Sinusitis, akute Pharyngitis, akute Tonsillitis, akute Laryngitis und Tracheitis, akute obstruktive Laryngitis (Krupp) und Epiglottitis,

- Grippe und Pneumonie,

- sonstige akute Infektionen der unteren Atemwege: akute Bronchitis, akute Bronchiolitis.

Im Vergleich zu Glasziou und Del Mar (2002) wird die akute Bronchitis hier als eine Erkrankung der unteren Atemwege definiert.

Im Gegensatz zum prävalenzorientierten ICD-System wurde 1985 speziell für die Klassifikation von Krankheiten in der Allgemeinmedizin die „International Classification of Primary Care" erstellt (Lamberts und Wood 1987). Diese neu geschaffene Einteilung ist stärker episoden-, bzw. prozessorientiert. Sie reflektiert somit eher die in der Allgemeinmedizin häufige Langzeitbehandlung von Patienten (Lamberts 1991). Nach ICPC (Lamberts et al. 1993) werden Atemwegsinfekte in folgende Untergruppen eingeteilt:

- R74 oberer Atemwegsinfekt,

- R75 akute Sinusitis,

- R76 akute Tonsillitis,

- R77 akute Laryngitis/Tracheitis,

- R78 akute Bronchitis.

Der ICPC sieht jedoch keine Klassifikation akuter Exazerbationen chronisch obstruktiver Lungenerkrankungen vor.

\subsection{Epidemiologie}

Die Erkältung ist die häufigste akute Erkrankung der industrialisierten Welt (Kirkpatrick 1996). Durchschnittlich erkrankt jeder Erwachsene 3-4-mal pro Jahr an einem akuten Atemwegsinfekt (Brede 1997). Dabei liegt die Krankheitsdauer zwischen 3 und 5 Tagen, gelegentlich auch länger. Durch die Häufigkeit von Infektionen bei Kleinkindern findet sich wahrscheinlich eine erhöhte 
Inzidenz von Atemwegsinfekten bei jungen Familien zwischen dem 20. und 29. Lebensjahr (Mähler 1996).

Schnur und Exner (1997) erfassten über einen Zeitraum von 2 Jahren alle Patienten mit akuten Atemwegsinfekten in ihrer Allgemeinarztpraxis. Etwa 60\% aller Patientenkontakte wegen eines akuten Atemwegsinfektes entfielen auf die Gruppe der 20- bis 50-Jährigen mit einem Krankheitsmaximum im 3. und 4. Lebensjahrzehnt und einem Abfall ab der 5. Dekade. Eine Häufung von Infektionen der Atemwege fand sich in den Übergangsjahreszeiten Herbst/Winter und Winter/Frühling, wobei $75 \%$ der Erkrankungen in den Monaten Januar bis März und September bis Dezember registriert wurden (Schnur und Exner 1997). Eine geringere Inzidenz fand sich in den Monaten Juni bis August. Somit konnte von saisonalen Erkrankungswellen gesprochen werden.

In der Häufigkeitsstatistik jährlicher Inanspruchnahmen von Ärzten nehmen Atemwegsinfekte eine führende Position ein. Kirkpatrick (1996) beschreibt Atemwegsinfekte mit ca. 10\% als den häufigsten akuten Beratungsanlass in seiner Allgemeinarztpraxis. Mähler (1996) gibt Zahlen von zum Teil über 50\% des Patientengutes in deutschen Allgemeinarztpraxen im Winter 95/96 an.

Auch in der Fehlzeitenstatistik nehmen Atemwegsinfekte eine führende Rolle ein. Sie sind mit 30\% der häufigste Grund für das Fernbleiben am Arbeitsplatz und von der Schule (Kirkpatrick 1996). Brede (1997) gibt in diesem Zusammenhang an, dass in Abhängigkeit vom Wetter zwischen $53 \%$ und $86 \%$ aller Krankmeldungen im Jahresverlauf auf akute Atemwegserkrankungen zurückzuführen sind.

Trotz des zumeist gutartigen Verlaufs stellen Atemwegsinfekte für das Gesundheitswesen in Deutschland eine erhebliche Belastung dar. So konnten Vollmer et al. (2000) unter Einbeziehung der Ausgaben für Diagnostik, Medikamente und Fehlzeiten der Arbeitnehmer 1997 folgende Kosten errechnen: akute Bronchitis 3,7 Mrd. DM, Rhinitis 0,8 Mrd. DM, Pneumonie 3,0 Mrd. DM und Influenza 5,1 Mrd. DM. Die Höhe dieser Kosten macht deutlich, dass sich gerade der Bereich der Atemwegsinfekte für Einsparungen im Gesundheitswesen anbietet (Schwabe 2002 c) 
und Untersuchungen über den Umgang mit Atemwegsinfekten in der hausärztlichen Praxis notwendig sind.

\section{3 Ätiologie}

95\% aller akuten Infektionen des oberen Respirationstraktes werden durch Viren verursacht (Adam et al. 1991). Die Autoren beschreiben die häufigsten Viren und die von ihnen verursachten Erkrankungen bzw. Symptome sowie den am stärksten betroffenen Personenkreis.

Tabelle 1: Virale Erreger und entsprechende Erkrankung/Symptomatik nach Adam et al. (1991, S.310)

\begin{tabular}{llll}
\hline Virusart & Virus & Erkrankung/Symptomatik & Betroffener Personenkreis \\
\hline Paramyxoviren & Parainfluenza & Laryngitis & Kinder \\
& Parainfluenza & Oberer Atemwegsinfekt & Kinder \\
Pneumonievirus & RS-Virus & Obere und untere Atemwege & Kinder, Jugendliche \\
Picornaviren & Coxsackie-Virus A & Respirationssymptomatik & \\
& Coxsackie-Virus B & Respirationssymptomatik & \\
& ECHO-Virus & Respirationssymptomatik & \\
& Enterovirus & Respirationssymptomatik & \\
& & Pneumonie & Kinder \\
Adenoviren & & Akute fieberhafte Pharyngitis & Junge Erwachsene \\
& & Akute Respirationskrankheit & Kinder, Personen mit \\
& & Pneumonie & Abwehrschwäche \\
Herpesviren & & Pertussis-like syndrome & Kinder \\
\hline
\end{tabular}

Bei 5-10\% der Erkrankten handelt es sich um primär bakterielle Infektionen (Adam et al. 1991). Die ätiologisch wichtigsten Bakterien sind demnach:

- Streptococcus pneumoniae (Pneumokokken),

- Haemophilus influenzae (vor allem unbekapselte Stämme),

- beta-hämolysierende Streptokokken (überwiegend Streptococcus pyogenes, serologische Gruppe A), 
- Staphylococcus aureus,

- Moraxella catarrhalis.

Nach Meinung von Adam et al. (1991) können diese Bakterien auch für eine bakterielle Superinfektion verantwortlich sein, die sich ihrer Ansicht nach häufig in eitrigen Sputumbeimengungen manifestiert. Winther et al. (1984) konnten jedoch anhand von Bakterienkulturen zeigen, die sie Patienten an verschiedenen Krankheitstagen während eines oberen Atemwegsinfektes aus dem Bereich des Nasenrachenraumes entnahmen, dass für das aufgetretene eitrige Sekret keine bakterielle Infektion ursächlich war. Sie sehen eitriges Sekret als direkte Folge einer viralen Infektion. Bormann et al. (2003) sammelten 232 Sputumproben von Patienten mit akutem produktiven Husten in 36 Hausarztpraxen und werteten sie mikrobiologisch aus. Dabei konnte eine bakterielle Infektion nur bei $12 \%$ der Patienten als gesichert angesehen werden. Lediglich für akute Exazerbationen chronischer Lungenerkrankungen konnte bislang ein Zusammenhang zwischen eitrigem Sputum und positiven Bakterienkulturen nachgewiesen werden (Stockley et al. 2000). Lag eitriges Sputum vor, war bei $84 \%$ der Patienten eine positive bakterielle Kultur nachweisbar, während dies nur bei 38\% der Patienten mit nicht-eitrigem Sputum der Fall war. Die klinische Angabe von eitrigem Sputum sprach auf der Basis dieser Daten mit einer 94,4\%-Sensitivität und 77,0\%Spezifität für das Vorliegen einer erhöhten Bakterienanzahl im Sputum von COPD-Patienten mit akuter Exazerbation. Entsprechend den Daten von Winther et al. (1984) dürfen diese Daten jedoch nicht auf akute obere Atemwegsinfekte ohne COPD übertragen werden.

\subsection{Evidenzbasierte Therapie}

Eine Volksweisheit lautet folgendermaßen: „Eine Erkältung, mit Medikamenten behandelt, dauert 7 Tage und ohne Medikamente eine Woche”. Im folgenden Kapitel wird dargestellt, inwieweit diese Aussage dem aktuellen Stand der Forschung entspricht. Grundlage ist eine Literatursuche nach Reviews und Metaanalysen zu den Themen oberer Atemwegsinfekt, akute Bronchitis, akute Pharyngitis, akute Sinusitis und akute Tonsillitis in Medline und in der Cochrane Library (Stand März 2003). 
Obwohl Antibiotika keine antivirale Wirkung aufweisen und in Kapitel 1.3 gezeigt wurde, dass die Mehrzahl der oberen Atemwegsinfekte viral bedingt sind, ist die Anwendung dieser Präparate weit verbreitet (Heikkinen und Järvinen 2003). Zwei Metaanalysen (Arroll und Kenealy 2003, Fahey et al. 1998) konnten bei oberen Atemwegsinfekten keine Veränderungen im klinischen Resultat oder in der Rate an Komplikationen durch die Gabe von Antibiotika nachweisen. Minimale Effekte fanden sich bei der Behandlung der akuten Bronchitis, Pharyngitis und Sinusitis. Demnach reduzierten Antibiotika bei akutem Husten geringfügig die Rate der Patienten, die bei einer Folgekonsultation über Husten klagten (Smucny et al. 2003). Bei akuter Pharyngitis konnte ein Review eine Reduktion des Risikos für das rheumatische Fieber nachweisen (Del Mar et al. 2003). Bei akuter Sinusitis reduzierte die Antibiotikagabe die Symptome signifikant verglichen mit der Plazebogabe (De Ferranti et al. 1998, Williams et al. 2003). Nicht unterschätzt werden darf jedoch, dass den geringen positiven Effekten der Antibiotikagabe z. T. erhebliche Nebenwirkungen gegenüber stehen und Resistenzentwicklungen beschleunigt werden.

Die Behandlung mit Virostatika wurde auf Grund der viralen Genese von Atemwegsinfekten in einer Metaanalyse von Jefferson und Tyrrell (2003) untersucht. Interferon-Nasenspray hatte eine präventive Wirkung auf experimentell erzeugte Atemwegsinfekte und in geringerem Ausmaß auch auf „natürliche“ Infekte. Die hohe Rate an Nebenwirkungen stand jedoch in keinem sinnvollen Verhältnis zum geringen Wirkungsgrad. Andere, z. T. noch experimentelle Substanzen (Impulsin, Pleconaril) wiesen ein wesentlich besseres Verhältnis von Wirkung und Nebenwirkung auf, sind jedoch weder zugelassen noch auf Grund der zu erwartenden Kosten als sinnvoll zu bezeichnen.

Die Gabe von B-Agonisten konnte die Dauer des Hustens geringfügig verkürzen, sodass mehr Patienten bei der Anwendung von ß-Agonisten nach 7 Tagen hustenfrei waren als bei der Einnahme von Erythromycin (Hueston 1994) oder der Inhalation eines Placebos (Melbye et al. 1991). Neuere Studien oder Reviews zu diesem Thema liegen nicht vor.

Gemäß eines Cochrane Reviews von Douglas et al. (2003) konnte gezeigt werden, dass Vitamin C (1 g täglich) die Dauer der Symptomatik bei undifferenzierten Erkältungen abkürzen konnte. 
Der günstige Effekt fiel jedoch mit einem halben Tag Symptomverkürzung gering aus und könnte laut den Autoren möglicherweise durch einen Publikationsbias bedingt sein.

Zwei Reviews konnten zeigen, dass die orale Gabe von Zink (Glukonat oder Acetat) zu keiner relevanten Symptomreduktion führte (Marshall 2003, Jackson et al. 2000). Für die intranasale Anwendung von Zink liegen widersprüchliche Daten vor (Hirt et al. 2000).

Die Beurteilung des Effektes von Echinacea wird dadurch beeinträchtigt, dass es mehr als 200 Präparate unterschiedlicher Zubereitung und Zusammensetzung gibt und die bisher erschienenen Studien methodische Mängel aufweisen. Kürzlich wurde erstmals eine randomisierte Kontrollstudie zur Anwendung von Echinacea in einem Medline-gelisteten Journal veröffentlicht. Diese Studie konnte keinen Effekt in der Akutbehandlung zeigen (Barrett et al. 2002). Dies steht im Einklang mit den Ergebnissen des aktuellen Cochrane Reviews zu diesem Thema. Demnach liegt für Echinacea in der Behandlung eines akuten Infektes keine Evidenz vor. Lediglich im Rahmen der Infektprophylaxe konnte eine reduzierte Infektrate auf der Basis von drei Kontrollstudien ermittelt werden (Melchart et al. 2003).

$\mathrm{Zu}$ Dampfinhalationen konnte ein Cochrane Review identifiziert werden, das eine geringfügige Besserung der Symptome nachweisen konnte. Immerhin konnten keine Nebenwirkungen beobachtet werden (Singh 2003).

In einem Cochrane-Review zu abschwellenden Mitteln konnten Taverner et al. (2003) einen symptomatischen Kurzzeiteffekt nachweisen. Für eine länger andauernde Verwendung gab es keine Evidenz.

Der positive, aber geringe Effekt von Antihistaminika bei der Linderung der Symptome der laufenden Nase und des Niesens wurde in zwei Metaanalysen (D`Agostino et al. 1998, Schroeder und Fahey 2003) beschrieben.

In einem systematischen Review basierend auf 12 randomisierten Kontrollstudien wurde die positive Wirksamkeit von Analgetika und antiinflammatorischen Medikamenten auf die 
Linderung von Halsschmerzen dargestellt (Thomas et al. 2000). Eine randomisierte Kontrollstudie zeigte einen positiven Effekt von steroidhaltigem Spray bei der Behandlung der akuten Sinusitis (Meltzer et al. 2000).

\subsection{Arzneiverordnungen bei akuten Atemwegsinfekten in Deutschland}

Wie in Kapitel 1.4 beschrieben, gibt es für viele der angewendeten Wirkstoffe bei der Therapie akuter oberer Atemwegsinfekte nur eine geringe Evidenz. Dennoch machen sie einen großen Teil an den Gesamtarzneiverordnungen aus (Schwabe 2002 a).

Antitussiva und Expektoranzien standen mit 40,7 Millionen Verordnungen und einem Umsatz von 264,5 Millionen Euro 2001 an 5. Stelle der Indikationsgruppen aller Arzneimittel (Schwabe 2002 a). Dies stellt jedoch einen erheblichen Rückgang, verglichen mit den Vorjahren, dar. So wurden 1997, im Jahr der Datenerhebung der vorliegenden Studie, noch 60,3 Millionen Präparate mit einem Umsatz von 810,7 Millionen DM aus dieser Gruppe verordnet (Schwabe 1999 a).

Auch bei den Rhinologika/Sinusitismitteln ist es in den letzten Jahren zu einem Rückgang der Verordnungen gekommen. 2001 lagen sie bei 20,3 Millionen pro Jahr (Umsatz 107,2 Millionen Euro), verglichen mit 29,0 Millionen Verordnungen und einem Umsatz von 232,6 Millionen DM im Jahr 1997 (Schwabe 1999 a, Schwabe 2002 a).

Nahezu unverändert stellt sich jedoch die Zahl der Antibiotikaverordnungen dar. Nach Schwabe (2002 a) wurden im Jahr 2001 42,3 Millionen Präparate aus der Gruppe der Antibiotika und Antiinfektiva verschrieben. Diese Zahl lag sogar noch über der von 1997 mit 40,9 Millionen Verordnungen (Schwabe 1999 a). Antibiotika lagen inzwischen an 3. Stelle, was die Häufigkeit ihrer Verordnung betrifft.

Einige Studien aus verschiedenen Ländern zeigten, dass bei der Behandlung von Atemwegsinfekten besonders Antibiotika zu häufig und ohne Berücksichtigung der Ätiologie verordnet wurden (de Melker und Kuyvenhoven 1994, Mainous III et al. 1996, Gonzales et al. 1997, Hummers-Pradier et al. 1999). Ferner ist die Effektivität von Expektoranzien in der 
Diskussion. Sie gehören zu den umstrittenen Arzneimitteln, die nach § 34 Abs.1 SGB V von der Arzneimittelversorgung ausgeschlossen sind (Schwabe 2002 c).

Dies führt zu der Vermutung, dass bei den Verordnungen andere Faktoren als die Wirksamkeit dieser Medikamentengruppen eine Rolle spielen.

\subsection{Mögliche Einflussfaktoren auf das Verordnungsverhalten der Ärzte}

Zwischen 60\% und 75\% aller Patientenkontakte führen laut Palmer (1990) in der Allgemeinarztpraxis zur Verordnung eines Arzneimittels. Diese Entscheidung zur Verordnung eines Präparates wird daher offensichtlich weniger von medizinischen Notwendigkeiten, als von sekundären, z.T. krankheitsunabhängigen Faktoren bestimmt. Im englischen Sprachraum werden diese Faktoren auch als „,non-pharmacological basis of therapeutics" bezeichnet (Kochen 1988).

Kochen (1994) nennt verschiedene Gründe für die Verordnung von Arzneimitteln in der Allgemeinarztpraxis:

- symptomatische Linderung von Beschwerden,

- Erwartungen des Patienten,

- vom Patienten gewünschte Aufrechterhaltung der „Krankenrolle",

- Beendigung der Konsultation,

- ärztliches Sicherheitsbedürfnis,

- Heilung von Krankheiten.

Die Erwartung des Patienten stellt hierbei einen starken Einflussfaktor dar. So konnten Cockburn und Pit (1997) zeigen, dass Patienten, die eine Verschreibung eines Arzneimittels erwarteten, verglichen mit solchen ohne diese Erwartung eine etwa 3fach erhöhte Wahrscheinlichkeit hatten dieses auch zu erhalten (Odds ratio (OR) 2,9). Sie war sogar etwa um das 10fache erhöht, wenn der behandelnde Arzt vermutete, der Patient erwarte ein Rezept (OR 10,1). Diese Einstellung der

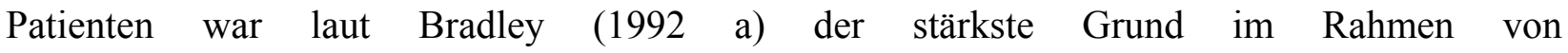
Medikamentenverordnungen eine Entscheidung $\mathrm{zu}$ finden und rangiert deutlich vor der medizinischen Angemessenheit. Offensichtlich besteht hier jedoch eine Diskrepanz bei der 
Wahrnehmung der Patientenerwartungen durch die behandelnden Ärzte, wie eine Untersuchung von Himmel et al. (1997) bestätigen konnte. So nahmen Ärzte in dieser Untersuchung die Erwartungen ihrer Patienten nur in 41\% der Fälle korrekt wahr. Diese Beobachtungen werden gestützt durch eine Studie aus dem Jahr 2000. Von Ferber (2000) konnte hier bei 635 befragten Patienten zeigen, dass diese in nur 20\% der Fälle ein Rezept erwarteten, jedoch zu 55\% eines erhielten.

Webb und Loyd (1994) fanden eine deutlich geringere Differenz. In ihrer Untersuchung von 1080 Konsultationen durch 12 Allgemeinärzte erwarteten 51\% der Patienten eine Verordnung gegenüber 55\% tatsächlicher Verordnungen. Insgesamt besteht bei den behandelnden Ärzten die Neigung die Patientenerwartungen an eine Verschreibung überzuinterpretieren (Britten 1995).

Eine Ursache der fehlenden Kongruenz der Patientenerwartung einerseits und ihrer Wahrnehmung durch den Arzt andererseits ist u.a. ein Kommunikationsdefizit (Britten et al. 2000). So konnten Butler et al. (1998) in einer britischen Studie zeigen, dass nur eine von 21 interviewten Allgemeinärzten die Patienten bezüglich ihrer Erwartungen und Wünsche explizit befragt hatte. Offensichtlich gab es hier auf beiden Seiten eine größere Hemmschwelle. Dabei steht aus Sicht der Patienten das Gespräch mit dem Arzt an erster Stelle der Patientenerwartungen an den Arztbesuch (von Ferber 2000), und zwar noch vor diagnostischen und therapeutischen Maßnahmen und der allgemeinen Untersuchung. Nach einer Studie von Kravitz et al. (1994) bei 304 ambulanten internistischen Patienten wurde deren Erwartung dadurch am meisten enttäuscht, dass ihre eigene Meinung zur Behandlung zu wenig oder gar nicht diskutiert wurde (38\% der Nennungen). Himmel et al. (1997) empfehlen daher, die Betroffenen direkt zu ihren Erwartungen zu befragen. Dieses Vorgehen führte nach ihren Ergebnissen zu keinem Anstieg des Arzneikonsums, da eine gerechtfertigte und begründete Ablehnung eines Arzneiwunsches bei der Mehrheit der Ratsuchenden keine negative Einschätzung des Arztbesuches zur Folge hatte. Ein derartiges Procedere wird jedoch nur von einer Minderheit der Ärzte tatsächlich angewendet, wie in der qualitativen Studie von Butler et al. (1998) mittels Arzt- und Patienteninterviews bei der Behandlung von Halsschmerzen gezeigt wurde. Nur eine Ärztin von 21 Interviewten befragte ihre Patienten selbst nach deren 
Erwartungen. Zwei Ärzte bezeichneten dieses Vorgehen sogar als belastend für die ArztPatienten-Beziehung.

Neben dem fehlenden Gespräch über die Patientenerwartung ist sicherlich auch eine Diskrepanz in der Begrifflichkeit zu suchen. So wird die Patientenerwartung aus ärztlicher Sicht weiter gefasst im Sinne einer Zufriedenheit mit der Konsultation. In dem Zusammenhang sind u.a. Aspekte wie die symptomatische Linderung der Beschwerden, das Vermitteln einer „Ernsthaftigkeit" der Symptome als Bestätigung der Notwendigkeit der Konsultation und das Bedürfnis den Patienten etwas mitzugeben zu nennen (Kochen 1994). Letztlich sind dies Bemühungen, den Patienten an die Praxis zu binden. Zumeist wird dies mit dem Mittel der Arzneiverordnung verknüpft, was sich in der bekannt hohen Rate an Verordnungen widerspiegelt (Himmel et al. 1997).

Die Verordnung von Medikamenten entspricht in diesem Zusammenhang dem einer „Pseudoplazebo"-Verordnung (Kochen 1994). Hierunter versteht man die Anwendung von Präparaten trotz fehlender, dokumentierter Wirksamkeit, einer annähernden Nebenwirkungsfreiheit sowie einer Kostengünstigkeit des Medikamentes.

Dabei ist wohl vielen Ärzten bewusst, wie zweifelhaft dieses Verordnungsverhalten ist. So zeigte Bradley (1992 b) in einer qualitativen Studie zum Verordnungsverhalten von Allgemeinärzten, dass die Verschreibung von Medikamenten gerade bei der Diagnose eines Atemwegsinfektes mehr als bei anderen Diagnosen zu einem hohen Maß an „Unbehagen" (engl. „discomfort”) bei den Ärzten führte. Hierzu trugen vor allem Ängste vor Nebenwirkungen (51,4\% Nennungen) und die Kosten (41,4\%) bei. Die Zunahme von Antibiotikaresistenzen spielte nur eine untergeordnete Rolle (17,1\%).

Die Konzentration der Ärzte auf das Mittel der Arzneiverordnung stellt dabei jedoch eine Reduktion dar, die von Patientenseite keineswegs immer so gewünscht wird. Nach SanchezMenegay et. al. (1992) wurde von 237 Patienten mit oberen Atemwegsinfekten am häufigsten als Ziel des Arztbesuches genannt eine Diagnose zu erhalten (57\%). Auch in dieser Studie erhielten mehr Patienten eine Medikamentenverordnung als von Patientenseite erwartet. 
Sanchez-Menegay et al. (1992) ermittelten ebenfalls, dass die Patientenzufriedenheit mit einem Arztbesuch bei oberen Atemwegsinfekten mit dem Maß an Beruhigung durch den Arzt stärker korrelierte als mit der erhaltenen Medikation. Diesen Sachverhalt fasst Kochen (1994) in dem Satz zusammen, dass Allgemeinärzte bei ihrem Verordnungsverhalten bedenken sollten, dass der häufigste Grund zur Konsultation nicht der Wunsch nach einer Behandlung, sondern nach einer Erklärung der Beschwerden ist.

\subsection{Komplikationen}

Akute Atemwegserkrankungen haben für den Allgemeinarzt/Hausarzt eine besondere Bedeutung, da er neben dem Kinderarzt am häufigsten mit diesem Krankheitsbild konfrontiert wird (Mähler 1996). Ihm kommt die schwierige Aufgabe $\mathrm{zu}$ zwischen Erkrankungen mit hoher Selbstheilungstendenz und abwendbar gefährlichen Verläufen zu unterscheiden (Pillau und Abholz 1992). Es gibt jedoch nur wenige Daten über die Häufigkeiten von Komplikationen bei Atemwegsinfekten, was durch eine Medline-Suche (Stand März 2003) bestätigt wurde. Es konnten nur zwei relevante Studien aus dem hausärztlichen Bereich herangezogen werden, die den Krankheitsverlauf als Studienziel definierten. So fand sich für die akute Sinusitis eine Studie (Stalman et al. 2001), bei der die Krankheit ohne relevante Komplikationen verlief (177 Patienten). Eine weitere Studie bei 316 Patienten mit akuter Bronchitis konnte ebenfalls keine Komplikationen nachweisen (Macfarlane et al. 2001).

Das rheumatische Fieber stellt die wohl gefürchtetste Komplikation der akuten Tonsillitis dar. Das Risiko nach einer unbehandelten, durch B-hämolysierende Streptokokken der Gruppe B bedingten Infektion an einem rheumatischen Fieber zu erkranken ist mangels aktueller Daten jedoch unklar (Olivier 2000). Auffällig erscheint jedoch, dass die Inzidenz des rheumatischen Fiebers bereits vor Einführung des Penicillins in Europa und Nordamerika stark abgenommen hatte (Flückinger 2001, Olivier 2000) und im Jahr 1930 mit etwa 2 Fällen pro 100.000 Infekten angegeben wurde (Bisno 1991). Die Inzidenz in entwickelten Ländern beträgt derzeit 0,5/100000 Kinder im Schulalter (Olivier 2000). 


\subsection{Fragestellung und Zielsetzung der Untersuchung}

Die vorliegende Arbeit beschreibt die Diagnostik und Therapie von Atemwegsinfekten in der Allgemeinarztpraxis. Dabei wurde die Vorgehensweise der Fachärzte für Allgemeinmedizin bei Patienten dokumentiert, die mit den Symptomen eines Atemwegsinfektes vorstellig wurden. Hierbei interessierte die alltägliche Praxis, ohne dass nach Möglichkeit Einfluss auf Diagnostik und Therapie genommen werden sollte. Aus diesem Grund wurde die Methode der teilnehmenden Beobachtung gewählt, auf deren Besonderheit in Kapitel 4.1 eingegangen wird. Weiterhin wurden mögliche Einflussfaktoren (Symptome, anamnestische Hinweise etc.) auf die diagnostische Klassifizierung und die therapeutische Entscheidungsfindung erfasst. Die Ergebnisse sollen dazu beitragen, überflüssige Diagnostik und Therapie zu vermeiden, aber gleichzeitig abwendbar gefährliche Verläufe zu erkennen. 


\section{Material und Methoden}

Es wurde eine Erhebung zur Diagnostik und Therapie von Atemwegsinfekten bei niedergelassenen Fachärzten für Allgemeinmedizin durchgeführt. $\mathrm{Zu}$ diesem Zweck fand eine jeweils eintägige Hospitation in 30 Allgemeinarztpraxen statt, um die Vorgehensweise möglichst vieler Ärzte zu erfassen.

Die vorliegende Studie wurde als teilnehmende Beobachtung (engl.: participant observation) durchgeführt, deren Charakteristikum entweder die retrospektive Dokumentation oder aber die Verwendung von technischen Hilfsmitteln wie Tonbändern oder Videokameras ist (Buser und Kaul-Hecker 1991). Die Untersuchung stellte insofern eine Abweichung von dieser Methode dar, als dass sie unter Zuhilfenahme eines Dokumentationsbogens zeitgleich mit der Konsultation das Anamnesegespräch ebenso wie die körperliche Untersuchung und die anschließend verordnete Therapie erfasste. Auf die spezifischen Vor- und Nachteile dieser Form der Datenerhebung wird in Kapitel 4.1 näher eingegangen.

\subsection{Auswahl der Ärzte}

An der Studie nahmen 30 Fachärzte für Allgemeinmedizin im Zeitraum von März bis April 1997 teil. Um eine möglichst heterogene Gruppe von Medizinern $\mathrm{zu}$ erfassen, wurden 16 Hospitationen in Hildesheim und 14 im Raum Brilon durchgeführt.

Hildesheim ist eine Großstadt in Niedersachsen mit ca. 106000 Einwohnern (Stand Dezember 1997). Die Stadt Brilon liegt im Hochsauerlandkreis. Ihre Einwohnerzahl und die der zugehörigen Gemeinden beträgt ca. 28000. Brilon wird somit als mittlere kreisangehörige Stadt bezeichnet. Somit wurden Ärzte einer Großstadt und ihre Kolleginnen und Kollegen aus einem eher ländlichen Bereich erfasst. 
Die Adressen der niedergelassenen Fachärzte für Allgemeinmedizin wurden freundlicherweise von der Kassenärztlichen Vereinigung Hildesheim und der Kassenärztlichen Vereinigung Westfalen-Lippe auf Anfrage zur Verfügung gestellt.

Die Auswahl beschränkte sich in Hildesheim auf die Stadt, in Brilon wurden auch Ärzte der umliegenden Dörfer in die Studie einbezogen. Mit allen dort niedergelassenen Fachärzten für Allgemeinmedizin wurde zunächst schriftlich Kontakt aufgenommen. Dabei wurde das Thema der Studie vorgestellt mit der Bitte, an der Erhebung teilzunehmen und eine eintägige Hospitation in ihrer Praxis zu ermöglichen. Die angeschriebenen Ärzte wurden gebeten ein vorgefertigtes Antwortschreiben per Post oder per Fax an die Abteilung zurückzusenden. Erklärte sich ein Arzt bereit an der Studie mitzuwirken wurde er zwecks weiterführender Information und genauer Terminabsprache telefonisch kontaktiert.

\subsection{Zeitpunkt der Datenerhebung}

Infekte der Atemwege treten bevorzugt in der nasskalten Jahreszeit auf. Ein Morbiditätsgipfel kann insbesondere beim Übergang vom Herbst zum Winter und vom Winter zum Frühjahr beobachtet werden (Schnur und Exner 1997). Infekte zum Ausgang des Winters zeichnen sich gewöhnlich durch einen schwereren Verlauf, häufigere Komplikationen und längere kreislaufbedingte Komplikationen als in den übrigen Monaten aus (Hamm H 1988).

Als Zeitpunkt für die Hospitationen wurden die Monate März und April 1997 ausgewählt, da in diesem Zeitraum von einer erhöhten Inzidenz bzw. Prävalenz von Atemwegsinfekten in den Praxen ausgegangen werden konnte.

\subsection{Ablauf der Hospitation}

Die Hospitationen dauerten einen Tag. Vor Beginn der Sprechstunde erfolgte eine Vorstellung der eigenen Person, der verantwortlichen Abteilung und der Studie bei dem besuchten Arzt und dessen Personal mit der Bitte an allen Konsultationen teilnehmen zu dürfen. 
Stellte sich ein Patient mit den Symptomen eines Atemwegsinfektes beim Arzt vor, wurde dieser vor der Dokumentation mündlich um sein Einverständnis gebeten an der Studie teilzunehmen. Dabei wurde ihm versichert, dass die Datenverarbeitung anonym bleibt.

Die Dokumentation von Hausbesuchen und Kontakten, die ausschließlich beim Abholen eines Rezeptes mit dem ärztlichen Hilfspersonal entstanden, wurden in die Studie nicht aufgenommen. Patienten unter 14 Jahren fanden keine Berücksichtigung.

\subsection{Erhebungsinstrument}

Im Vorfeld der Hospitationen wurde eine Pilotstudie in einer Gemeinschaftspraxis über zwei Tage durchgeführt. Dabei wurde die Gestaltung und Anwendung des Dokumentationsbogens, die Durchführbarkeit der Dokumentation während der Konsultation und die Akzeptanz der Patienten und der Ärzte gegenüber der Erhebung geprüft. Es ergaben sich keine Schwierigkeiten während der Hospitation von Seiten der Patienten und der Ärzte. Der Dokumentationsbogen musste in wenigen Punkten noch ergänzt werden.

Während der Hospitation fanden 2 Dokumentationsbögen Verwendung (siehe Anhang). Der Erste bezog sich ausschließlich auf den Arzt und seine Praxis. Dabei handelte es sich um geschlossene Fragen zu Alter und Geschlecht des Arztes, Dauer der Niederlassung, evtl. erworbene Zusatzbezeichnungen, Scheinzahl pro Quartal, Tätigkeit in Einzelpraxis, Praxisgemeinschaft oder Gemeinschaftspraxis,

Zur Dokumentation der einzelnen Patientenkontakte wurde ein 2. Dokumentationsbogen erstellt, der es ermöglichen sollte das Anamnesegespräch, die körperliche Untersuchung, die Diagnosestellung und die Therapie möglichst zeitsparend zu erfassen (siehe Anhang). Dies geschah über ankreuzbare Auswahlfelder, die entsprechend der Schilderung von Symptomen, der Durchführung von Untersuchungen, der gestellten Diagnose und der empfohlenen Therapie angekreuzt werden konnten. Weitere freie Felder für Dauermedikation, Selbstmedikation, Eigentherapie, Untersuchungsbefunde und verordnete bzw. empfohlene Medikamente sowie für weitere Ergänzungen der Auswahlfelder standen zur Verfügung. Hierzu wurde auf Vorgaben aus 
dem Lehrbuch zu Anamnese und Befund von Dahmer (1988) sowie auf das Lehrbuch „Allgemeinmedizin“ von Kochen (1992) und den „Praxisleitfaden Allgemeinmedizin“ von Schmidt et al. (1996) zurückgegriffen.

Der 2. Dokumentationsbogen enthielt für die dokumentierten Patientenkontakte folgende Angaben:

1. Alter, Versicherungsart und Beruf des Patienten,

2. Patientenanamnese zum Atemwegsinfekt,

3. durchgeführte Selbstmedikation und Eigentherapie,

4. Raucheranamnese,

5. durchgeführte körperliche Untersuchungen und deren Befund,

6. zusätzliche apparative Diagnostik, Überweisung zum Spezialisten, Überweisung ins Krankenhaus,

7. medikamentöse Therapie, Dauermedikation, Verordnungspraxis (Privatrezept, Kassenrezept, ärztliches Muster, Empfehlung zum Selbstkauf) sowie evtl. Medikamentenwünsche,

8. zusätzliche Empfehlungen, Arbeitsunfähigkeitsbescheinigung und Wiedereinbestellung des Patienten,

9. bei Folgekontakten Angaben zur initialen Therapie.

Die unter Punkt 1 und Punkt 9 genannten Angaben wurden aus der Dokumentation der Ärzte übernommen, die in Form von Karteikarten oder im Computer gespeichert vorlag. Der Beruf des Patienten wurde erfragt. Angaben zur durchgeführten Selbstmedikation, Eigentherapie und Raucheranamnese wurden nur dann dokumentiert, wenn der Patienten sie erwähnte oder der Arzt sie erfragte. Die Ärzte wurden gebeten die jeweiligen Untersuchungsbefunde und Diagnosen während oder nach der Konsultation mitzuteilen.

Da bei den teilnehmenden Ärzten die gestellten Diagnosen üblicherweise nicht nach der „International Classification for Primary Care“ (ICPC) (Lamberts et al. 1993) klassifiziert wurden, erfolgte die Diagnosestellung nach Rücksprache mit ihnen auf der Basis der Vorgaben des ICPC. Unter der Diagnose oberer Atemwegsinfekt wurden folgende Bezeichnungen zusammengefasst: 
- grippaler Infekt,

- Infekt der oberen Luftwege,

- (banale) Erkältung,

- virale Rhinitis,

- Virusinfekt der oberen Luftwege.

Auf Grund der fehlenden Kodiermöglichkeit des ICPC für akute Exazerbationen chronisch obstruktiver Lungenerkrankungen erhielt beim Vorliegen einer akuten Exazerbation die entsprechende chronisch obstruktive Lungenkrankheit den Zusatz ,akut exazerbiert”:

- R91 chronische Bronchitis akut exazerbiert

- R95 Lungenemphysem/COPD akut exazerbiert

- R96 Asthma bronchiale akut exazerbiert

Ferner trat das Problem auf, dass mehrere Diagnosen pro Patient gestellt wurden. In den Auswertungen mussten aus diesem Grund auch Erst- und Zweitdiagnosen berücksichtigt werden, sodass die prozentuale Aufteilung der Diagnosen mehr als 100\% ergab.

Eine Unterscheidung nach Erst- und Folgekontakten erschien sinnvoll, da die initial verordnete Therapie bei Folgekontakten zwar anhand der Unterlagen der Ärzte nachvollziehbar war, die Dokumentation von Symptomen und Untersuchungsbefunden jedoch häufig fehlte, sodass keine Aussagen zur Entscheidungsfindung der Ärzte zur bereits begonnenen Therapie gemacht werden konnten.

\subsection{Auswertungsverfahren}

Zur Auswertung der beiden genannten Dokumentationsbögen wurden zwei Datenbanken mit Hilfe einer PC-Version des Computer-Programms Fox-Pro Version 2.5 für Apple Macintosh erstellt. Die erste Datenbank erhielt die Angaben zu den besuchten Ärzten und deren Praxen. Die dokumentierten Patientendaten wurden anonymisiert in einer zweiten Datenbank gespeichert. 
Die gestellten Diagnosen wurden nach der Klassifikation des ICPC (International Classification for Primary Care) kodiert, die verordneten Medikamente gemäß des ATC-Codes (Anatomischtherapeutisch-chemische Klassifikation für den deutschen Arzneimittelmarkt) von Schwabe (1995) eingeteilt und kodiert.

Zur statistischen Auswertung stand das Programm StatView 4.1 (Abacus Concepts, Inc.) für Apple Macintosh zur Verfügung. Die Prüfung von Unterschiedshypothesen erfolgte mittels Chiquadrat-Vierfeldertest oder Mehrfeldertest (Signifikanzniveau: $\alpha=0,05$ ). Des Weiteren wurde der t-Test für unabhängige Stichproben nach Überprüfung der Normalverteilung mittels FTest verwendet (Signifikanzniveau: $\alpha=0,05$ ).

Um eine Assoziation der Verordnung von Husten- und Erkältungspräparaten und Antibiotika mit den Symptomen und Untersuchungsbefunden der Patienten zu untersuchen, wurde eine multiple logistische Regressionsanalyse mittels der Software SAS Version 8 durchgeführt. Hierzu kam das Verfahren der Rückwärtselimination (backward elimination) mit $\mathrm{p}<0,05$ zur Ausschlussgrenze zur Anwendung. Dasselbe Verfahren wurde weiterhin zur Darstellung einer Assoziation der 4 Hauptdiagnosen oberer Atemwegsinfekt, akute Sinusitis, akute Tonsillitis und akute Bronchitis mit den Symptomen und Untersuchungsbefunden der Patienten eingesetzt.

Da die meisten Variablen einen dritten Ausprägungswert („nicht erfragt oder nicht untersucht") enthielten, mussten die Variablen zur Berechnung dichotomisiert werden. Dazu wurde der Wert der Variablen auf Null gesetzt, wenn sie nicht erfragt oder untersucht wurden. Dem liegt die Überlegung zu Grunde, dass die Symptome vermutlich auch nicht vorlagen oder von den Patienten als besonders belastend empfunden wurden, wenn sie nicht genannt wurden. Weiterhin wurden nicht erhobene Befunde damit als unauffällig gewertet, da sie zur Diagnose- und Therapieentscheidung für den Arzt bei diesem individuellen Patienten keine Relevanz zu haben schienen.

Die Berechnung auf der Basis aller erhobenen Symptome und Befunde führte auf Grund der hohen Anzahl der Variablen zu keinem stabilen Regressionsmodell. Daher wurde die Anzahl der Variablen auf die jeweils 10 häufigsten Symptome und pathologischen Befunde der genannten 
Diagnosen beschränkt. Zur besseren Vergleichbarkeit mit anderen Studien wurden zudem die Variablen Alter (ausgedrückt als jünger oder älter als 50 Jahre) sowie der Raucherstatus hinzugefügt. Folgende Variablen wurden eingeschlossen: Alter, Raucher; die Symptome Abgeschlagenheit, Kopfschmerzen, Schnupfen, Heiserkeit, Halsschmerzen, Ohrenschmerzen, Schmerzen der Nasennebenhöhlen, Fieber, Husten, Vorliegen von Auswurf (zusätzlich differenziert in gelblich-grünen und weißlichen Auswurf); die Untersuchungsbefunde pathologischer Otoskopiebefund, pathologische Atem- und Nebengeräusche bei der Auskultation, pathologischer Rachenbefund (zusätzlich differenziert in gerötete Tonsillen, gerötete Rachenhinterwand und mit Stippchen belegte Tonsillen), pathologische Befunde bei der Palpation der Halslymphknoten oder der Nasennebenhöhlen. 


\section{Ergebnisse}

\subsection{Rücklauf der Anschreiben}

Insgesamt wurden 44 Fachärzte für Allgemeinmedizin im Stadtgebiet Hildesheim und 18 im Raum Brilon angeschrieben und um die Ermöglichung einer eintägigen Hospitation gebeten. Es antworteten 29 der Hildesheimer und 15 der Briloner Ärzte. Von ihnen waren in Hildesheim 14 (48,3\%) und in Brilon 12 Ärzte (80,0\%) bereit, sich an der Studie zu beteiligen. Um die vorher festgelegte Zahl von $30 \mathrm{zu}$ besuchenden Ärzten zu erreichen wurden jeweils noch 2 Ärzte telefonisch gebeten an der Erhebung mitzuwirken.

\subsection{Beschreibung der Stichprobe und des Patientenkollektivs}

30 Fachärzte für Allgemeinmedizin waren in eine eintägige Hospitation involviert, von denen 16 in Hildesheim und 14 im Raum Brilon niedergelassen waren. Der Anteil der weiblichen Mediziner betrug insgesamt $16,7 \%$. 50,0\% der besuchten Ärzte praktizierten in einer Einzelpraxis, $40,0 \%$ in einer Gemeinschaftspraxis und 10,0\% in einer Praxisgemeinschaft. In Hildesheim beschäftigten 5 Ärzte einen Weiterbildungsassistenten. Bei den im Raum Brilon teilnehmenden Ärzten wurden keine Weiterbildungsassistenten ausgebildet.

12 Ärzte (9 in Hildesheim, 3 im Raum Brilon) führten eine oder mehrere Zusatzbezeichnungen (Sportmedizin, Chirotherapie, Naturheilverfahren, Psychotherapie, Umweltmedizin, Rettungsmedizin und Betriebsmedizin). 2 Ärzte einer Gemeinschaftspraxis besaßen die Anerkennung einer Schwerpunktpraxis für Diabetologie. In einer weiteren Praxis wurde ein Methadonprogramm für Drogenabhängige angeboten.

In Tabelle 2 erfolgt eine Differenzierung der Stichprobe und des Patientenkollektivs nach den Orten der Datenerhebung. Es ergaben sich keine signifikanten Unterschiede zwischen Hildesheim und dem Raum Brilon. Deshalb wurde im folgenden Ergebnisteil auf eine weitere Unterscheidung zwischen Daten, die in Hildesheim bzw. im Raum Brilon erhoben wurden, verzichtet. 
Tabelle 2: Differenzierung der Stichprobe und des Patientenkollektivs nach den Orten der Datenerhebung

\begin{tabular}{lcc}
\hline & Hildesheim & Raum Brilon \\
\hline Durchschnittsalter der Ärzte & 46,8 $( \pm 9,0)$ Jahre & $49,0( \pm 9,1)$ Jahre \\
Dauer der Niederlassung & 11,1 Jahre $( \pm 8,7)$ Jahre & $15,64( \pm 9,2)$ Jahre \\
Anteil der Atemwegsinfekte an der & $21,6 \%$, & $21,2 \%$ \\
Gesamtzahl der Konsultationen & & \\
Anzahl der Patienten & 154 & 119 \\
- davon weiblich & 86 & 54 \\
- davon männlich & 68 & 65 \\
Durchschnittsalter der Patienten & $42,4( \pm 18,9)$ Jahre & $42,7( \pm 21,1)$ Jahre \\
\hline
\end{tabular}

Eine Unterscheidung erfolgte jedoch zwischen Patienten, die sich erstmalig mit einem Atemwegsinfekt vorstellten (Erstkontakte) und denen, die bereits ein- oder mehrfach mit demselben Atemwegsinfekt die Sprechstunde aufgesucht hatten (Folgekontakte). Diese Unterscheidung war notwendig, um mögliche Veränderungen im Untersuchungs- bzw. Verordnungsverhalten der Ärzte bei einer erneuten Konsultation zu dokumentieren.

186 Patienten stellten sich erstmalig mit den Symptomen eines Atemwegsinfektes bei den jeweiligen Ärzten vor; 87 wurden als Folgekontakte dokumentiert, wobei 81 Patienten mit demselben Infekt zum 2. Mal erschienen. Bei 4 Patienten handelte es sich um den 3. Kontakt, 2 Patienten suchten zum 4. Mal den Arzt auf.

Abbildung 1 zeigt die Altersverteilung bei Erst- und Folgekontakten. Die Patienten bei einem Erstkontakt waren im Durchschnitt mit 40,2 $( \pm 19,2)$ Jahren jünger als die Patienten bei einem Folgekontakt 47,4 ( $\pm 20,4$ Jahre). Die Altersgruppe der 30- bis 39-Jährigen war bei Erst- und Folgekontakten am häufigsten vertreten. 


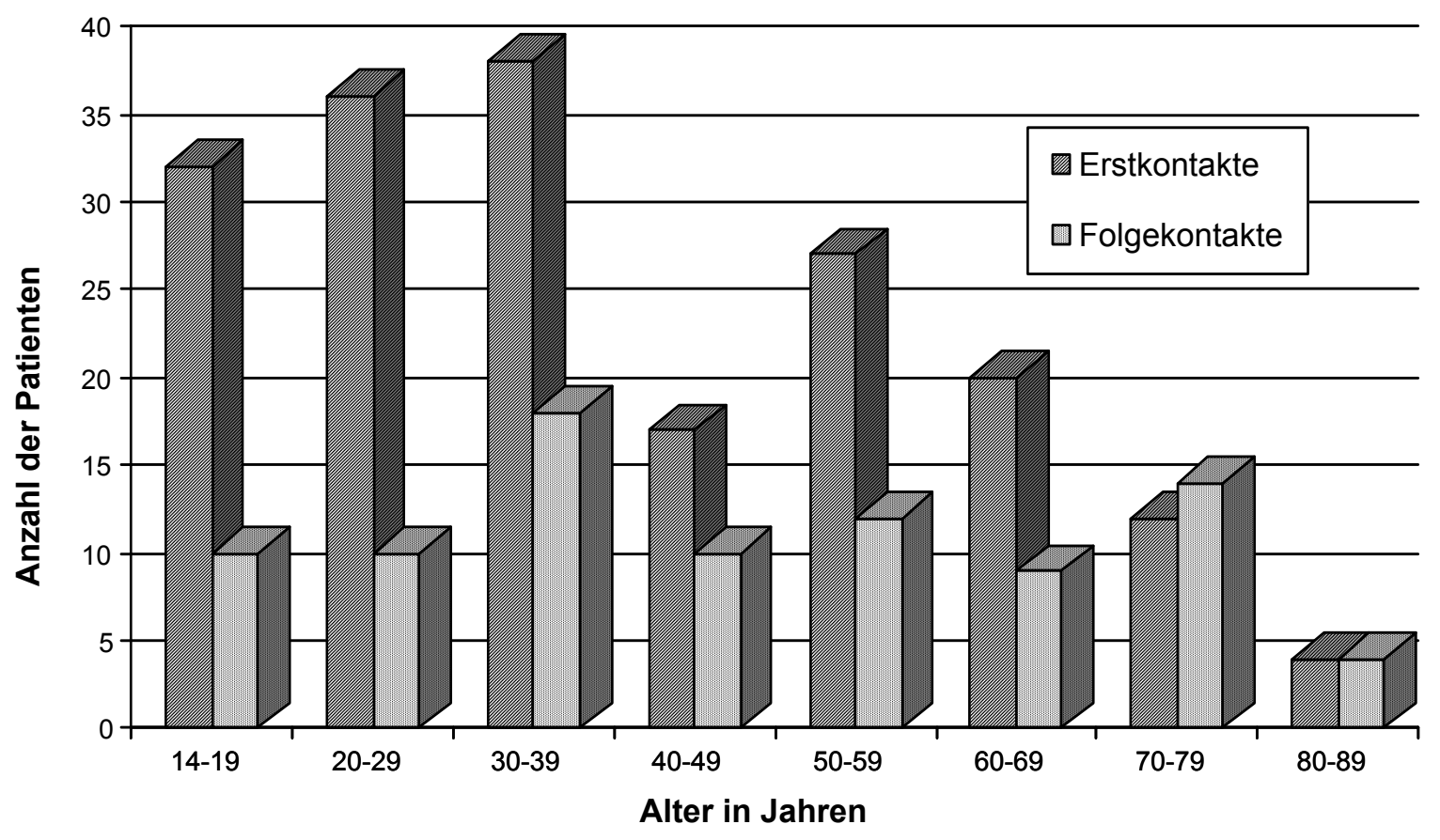

Abbildung 1: Altersverteilung bei Erst- und Folgekontakten

\subsection{Patientenanamnese}

\subsubsection{Krankheitsdauer bis zur Arztkonsultation}

Innerhalb der ersten 14 Tage nach Krankheitsbeginn konsultierten 93,6\% der Erstkontakte und 86,2\% der Folgekontakte den Arzt. Bei Erstkontakten suchten 52,7\% der Patienten den Arzt innerhalb der ersten 3 Tage auf. Weitere Häufungen waren am 7., 10. und 14. Tag zu beobachten. Der Mittelwert bis zur Erstkonsultation betrug 6,5 $( \pm 9,5)$ Tage. Bei den Folgekontakten stellten sich die meisten Patienten am 5., 7., 10. und 14. Tag nach Krankheitsbeginn erneut bei den Ärzten vor. 13,8\% der Kranken kamen nach dem 14. Tag. Der letzte Patient kam nach 40 Tagen Krankheitsdauer zur Folgekonsultation. Der Mittelwert bis zur Folgekonsultation, vom Krankheitsbeginn an gerechnet, betrug 10,5 $( \pm 8,1)$ Tage. 


\subsubsection{Geschilderte Symptome}

Erstkontakte schilderten im Durchschnitt 4,0 ( $\pm 1,7)$ Symptome, Folgekontakte 3,4 $( \pm 1,6)$. Beim Erstkontakt klagten 59,7\% der Patienten über 2 bis 4 Symptome, maximal wurden bei jeweils 2 Patienten 8 bzw. 9 Symptome geschildert oder vom Arzt erfragt. Nur ein Symptom wurde von $3,2 \%$ der Erstkontakte angegeben. $71,3 \%$ der Folgekontakte klagten über 2 bis 4 Symptome. $5,8 \%$ der Patienten beschrieben nur ein Symptom; maximal wurden von einem Patienten 9 angegeben oder erfragt. Tabelle 3 zeigt Art und Häufigkeit der vom Patienten geschilderten Symptome bei Erst- und Folgekontakten.

Tabelle 3: Art und Häufigkeit der geschilderten Symptome (Anzahl der Patienten absolut und in $\%)$

\begin{tabular}{lrrrr}
\hline \multicolumn{1}{c}{ Art der Symptome } & \multicolumn{2}{c}{ Erstkontakte } & \multicolumn{2}{c}{ Folgekontakte } \\
\hline Husten & 146 & $(78,5 \%)$ & 69 & $(85,2 \%)$ \\
Schnupfen & 105 & $(56,5 \%)$ & 30 & $(37,0 \%)$ \\
Halsschmerzen & 73 & $(39,3 \%)$ & 22 & $(27,2 \%)$ \\
Fieber & 61 & $(32,8 \%)$ & 8 & $(9,9 \%)$ \\
Kopfschmerzen & 57 & $(30,7 \%)$ & 16 & $(19,8 \%)$ \\
Abgeschlagenheit & 42 & $(22,6 \%)$ & 16 & $(19,8 \%)$ \\
Heiserkeit & 31 & $(16,7 \%)$ & 12 & $(14,8 \%)$ \\
Gliederschmerzen & 30 & $(16,1 \%)$ & 6 & $(7,4 \%)$ \\
Ohrenschmerzen & 24 & $(12,9 \%)$ & 11 & $(13,6 \%)$ \\
Schmerzen NNH & 14 & $(7,5 \%)$ & 10 & $(12,4 \%)$ \\
gastrointestinale Symptome & 12 & $(6,5 \%)$ & 4 & $(4,9 \%)$ \\
Kreislaufbeschwerden & 11 & $(5,9 \%)$ & 9 & $(11,1 \%)$ \\
Augenbeschwerden & 10 & $(5,4 \%)$ & 1 & $(1,2 \%)$ \\
Dyspnoe & 8 & $(4,3 \%)$ & 6 & $(7,4 \%)$ \\
Frieren & 7 & $(3,8 \%)$ & 2 & $(2,5 \%)$ \\
Schmerzen Halslymphknoten & 6 & $(3,2 \%)$ & 5 & $(6,2 \%)$ \\
Schwitzen & 5 & $(2,7 \%)$ & 1 & $(1,2 \%)$ \\
Schmerzen beim Atmen & 5 & $(2,7 \%)$ & 2 & $(2,5 \%)$ \\
Zahnschmerzen & 3 & $(1,6 \%)$ & 4 & $(4,9 \%)$ \\
Gelenkbeschwerden & 3 & $(1,6 \%)$ & 2 & $(2,5 \%)$ \\
Hörstörungen & 3 & $(1,6 \%)$ & 0 & \\
Exanthem & 2 & $(1,1 \%)$ & 2 & $(2,5 \%)$ \\
kardiale Symptome & 1 & $(0,5 \%)$ & 1 & $(1,2 \%)$ \\
Schüttelfrost & 1 & $(0,5 \%)$ & 0 & \\
Schmerzen beim Schnäuzen & 0 & & 2 & $(2,5 \%)$ \\
\hline & & & & \\
\hline
\end{tabular}




\subsubsection{Patientenanamnese zum Symptom Fieber}

Von den 69 Patienten, die über Fieber klagten, wurden 50 zur Fieberhöhe befragt. 17 gaben eine erhöhte Körpertemperatur zwischen $37,5-38,0^{\circ} \mathrm{C}$ an, 12 klagten über Fieber zwischen 38,1 und $38,5^{\circ} \mathrm{C}$. Bei 10 Patienten betrug die von ihnen gemessene Temperatur $38,6-39,0^{\circ} \mathrm{C}, 8$ maßen eine Temperatur von über $39,0^{\circ} \mathrm{C}$. 3 gaben anamnestisch Fieber an, obwohl die von ihnen gemessene Temperatur zwischen 36,9 und $37,4^{\circ} \mathrm{C}$ lag. Kein Patient wurde gefragt, wo er seine Körpertemperatur gemessen habe.

\subsubsection{Patientenanamnese zum Symptom Husten}

Husten war das von den Patienten am häufigsten geschilderte Symptom. 205 (78,5\% der Erstkontakte und 79,3\% der Folgekontakte) klagten darüber. Tabelle 4 zeigt, inwieweit bei diesen Patienten weitere Angaben zur Produktivität des Hustens, der Sputumfarbe und einer Verstärkung am Morgen oder in der Nacht vom ihnen selbst gemacht oder vom Arzt erfragt wurden. Die meisten Angaben bezogen sich dabei auf die Produktivität des Hustens und die Sputumfarbe. Hinweise zur Zunahme der Hustenintensität in der Nacht oder am Morgen lagen bei weniger als $20 \%$ bzw. 10\% der Patienten vor. Von 32 Patienten, die nach einer Verstärkung des Hustens in der Nacht gefragt wurden, gaben 96,9\% an darunter zu leiden.

Tabelle 4: Weiterführende Angaben zum Symptom Husten (Anzahl der Patienten absolut und in $\%)$

\begin{tabular}{lrlll}
\hline \multicolumn{1}{c}{ weiterführende Angaben } & \multicolumn{2}{c}{ Erstkontakte } & \multicolumn{2}{c}{ Folgekontakte } \\
\hline Produktivität des Hustens & 117 & $(80,1 \%)$ & 64 & $(92,8 \%)$ \\
- unproduktiv, trocken & 29 & $(24,8 \%)$ & 12 & $(18,8 \%)$ \\
- produktiv, feucht & 88 & $(75,2 \%)$ & 52 & $(81,3 \%)$ \\
Sputumfarbe & 64 & $(43,8 \%)$ & 38 & $(55,1 \%)$ \\
- weißlich & 18 & $(28,1 \%)$ & 15 & $(26,3 \%)$ \\
- gelblich -grün & 46 & $(71,9 \%)$ & 23 & $(60,5 \%)$ \\
Verstärkung des Hustens in der Nacht & 22 & $(16,4 \%)$ & 10 & $(14,5 \%)$ \\
Verstärkung des Hustens am Morgen & 5 & $(3,4 \%)$ & 5 & $(7,3 \%)$ \\
\hline
\end{tabular}




\subsubsection{Selbstmedikation und nichtmedikamentöse Therapie}

49 Patienten $(17,9 \%)$ machten Angaben zur Selbstmedikation oder wurden von den Ärzten bezüglich der Selbstmedikation befragt. Von 224 (82,1\%) wurde keine Anamnese zur Selbstmedikation erhoben. 41 (83,7\%) gaben an, eine Selbstmedikation durchgeführt zu haben, 8 Patienten verneinten diese Frage.

Die Gruppe der Husten- und Erkältungspräparate nahm mit 34 Selbstmedikationen den größten Anteil ein. Aus dieser Gruppe wurden Expektoranzien exklusive der Kombinationen mit Antitussiva mit 23 Medikationen und andere Erkältungspräparate wie z.B. Acetylsalicylsäure oder Paracetamol (9 Medikationen) am häufigsten eingenommen. Des Weiteren wurden Rhinologika (9 Medikationen), Halsschmerzmittel (5 Medikationen) und EchinaceaZubereitungen (2 Medikationen) angewendet.

Bezüglich der nichtmedikamentösen Therapie und der Verwendung von sogenannten Hausmitteln machten 33 Patienten (12,1\%) Angaben, 240 Patienten (87,9\%) wurden zu dieser Form der Therapie nicht befragt. Am beliebtesten waren verschiedene Präparate zur Inhalation wie z.B. Kamille, Kochsalz oder Chinaöl und zum Gurgeln wie z.B. Salbei, Kamille oder Essigsauretonerde. In Tabelle 5 erfolgt eine Darstellung der von den Patienten durchgeführten nichtmedikamentösen Therapien, wobei häufig mehrere Arten pro Patient geschildert wurden.

Tabelle 5: Arten der nichtmedikamentösen Therapie

\begin{tabular}{lc}
\hline Art der nichtmedikamentösen Therapie & Anzahl der Patienten \\
\hline Inhalation & 12 \\
Gurgeln & 9 \\
Bronchialtee & 5 \\
Halswickel & 2 \\
Einreibung mit ätherischen Ölen & 1 \\
\hline
\end{tabular}




\subsection{Durchgeführte Untersuchungen}

\subsubsection{Körperliche Untersuchungen und Untersuchungsbefunde}

Von den 273 Patienten wurden 271 körperlich untersucht (99,3\%), darunter alle Erstkonsultationen. Sowohl bei Erst- als auch bei Folgekontakten waren die Auskultation der Lunge und die Inspektion des Mund- und Rachenraums die am häufigsten durchgeführten Untersuchungen. Bei diesen fanden sich auch die meisten pathologischen Befunde. Insgesamt wurden bei Folgekontakten weniger Untersuchungen durchgeführt. Tabelle 6 gibt eine Übersicht über die von den Ärzten durchgeführten körperlichen Untersuchungen und deren Ergebnisse. Die Prozentangaben der Befunde beziehen sich auf die Patienten, bei denen diese Untersuchung erfolgte.

Tabelle 6: Körperliche Untersuchungen und Befunde (Anzahl der Patienten absolut und in \%)

\begin{tabular}{|c|c|c|c|c|}
\hline \multirow{2}{*}{$\begin{array}{l}\text { Durchgeführte Untersuchungen und } \\
\text { Untersuchungsbefunde }\end{array}$} & \multicolumn{2}{|c|}{ Erstkontakte } & \multicolumn{2}{|c|}{ Folgekontakte } \\
\hline & 159 & $(85,5 \%)$ & 47 & $(54,0 \%)$ \\
\hline a) Rachenhinterwand & & & & \\
\hline - pathologischer Befund & 142 & $(89,3 \%)$ & 38 & $(65,2 \%)$ \\
\hline \multicolumn{5}{|l|}{ b) Tonsillen } \\
\hline - pathologischer Befund & 139 & $(87,4 \%)$ & 37 & $(78,7 \%)$ \\
\hline Auskultation der Lunge & 153 & $(82,3 \%)$ & $\mathbf{7 0}$ & $(80,5 \%)$ \\
\hline \multicolumn{5}{|l|}{ a) Atemgeräusch } \\
\hline - pathologischer Befund & 26 & $(17,0 \%)$ & 8 & $(11,4 \%)$ \\
\hline \multicolumn{5}{|l|}{ b) Nebengeräusche } \\
\hline - pathologischer Befund & 46 & $(30,1 \%)$ & 27 & $(38,6 \%)$ \\
\hline Otoskopie & 63 & $(33,9 \%)$ & 10 & $(18,4 \%)$ \\
\hline \multicolumn{5}{|l|}{ a) äußerer Gehörgang } \\
\hline - pathologischer Befund & 5 & $(7,9 \%)$ & 1 & $(6,3 \%)$ \\
\hline \multicolumn{5}{|l|}{ b) Trommelfell } \\
\hline - pathologischer Befund & 9 & $(14,3 \%)$ & 7 & $(43,8 \%)$ \\
\hline Palpation der Nasennebenhöhlen & 60 & $(32,3 \%)$ & 23 & $(26,4 \%)$ \\
\hline - pathologischer Befund & 29 & $(48,3 \%)$ & 15 & $(65,2 \%)$ \\
\hline Palpation der Halslymphknoten & 60 & $(32,3 \%)$ & 23 & $(26,4 \%)$ \\
\hline - pathologischer Befund & 29 & $(48,3 \%)$ & 15 & $(65,2 \%)$ \\
\hline Perkussion der Lunge & 29 & $(15,6 \%)$ & 8 & $(9,2 \%)$ \\
\hline - pathologischer Befund & 0 & & 1 & $(12,5 \%)$ \\
\hline Stimmfremitus & 9 & $(4,9 \%)$ & 1 & $(1,2 \%)$ \\
\hline - pathologischer Befund & 0 & & 0 & \\
\hline
\end{tabular}


Zusätzlich wurde bei 22,0\% der Patienten eine Blutdruckmessung und bei 11,4\% der Patienten eine Auskultation des Herzens durchgeführt, für die jedoch kein konkreter Anlass bei der Konsultation erkennbar war. Die Messung der Körpertemperatur und die Untersuchung des Abdomens wurden bei entsprechend geäußerten Beschwerden vorgenommen.

\subsubsection{Labor- und apparative Diagnostik}

Labor- oder apparative Diagnostik kamen bei insgesamt 32 Patienten (11,7\%) zur Anwendung. Bei 6,6\% wurden Laboruntersuchungen angeordnet, wobei die Bestimmung der Leukozyten im Vordergrund stand. Bei 3,3\% der Patienten hielt der Arzt eine Lungenfunktionsprüfung für indiziert.

\subsection{3 Überweisung zu Spezialisten oder ins Krankenhaus}

7 Patienten (2,6\%) wurden zu anderen Fachärzten (Radiologe, Pulmonologe, HNO-Arzt) überwiesen, 4 Patienten beim Erst- und 3 Patienten beim Folgekontakt. Je 2-mal erfolgte eine Überweisung zum Röntgen der Nasennebenhöhlen, 2-mal zum Röntgen des Thorax und 3-mal zur Mit- und Weiterbehandlung. Kein Patient wurde in ein Krankenhaus eingewiesen.

\subsection{Diagnosestellung}

\subsubsection{Bei Erstkontakten}

In Tabelle 7 werden die insgesamt gestellten Diagnosen bezüglich der Erkrankungen der Atemwege und des Ohres bei Erstkontakten aufgeführt. Da Mehrfachdiagnosen möglich waren, ergab die Summe der Prozentangaben mehr als 100\%. Akute Erkrankungen der Atemwege machten einen größeren Anteil aus als akute Exazerbationen chronisch obstruktiver Lungenerkrankungen. 
Tabelle 7: Häufigkeit gestellter Diagnosen in Erst- oder Zweitdiagnose bei Erstkontakten

\begin{tabular}{lcc}
\hline Diagnose nach ICPC & $\begin{array}{c}\text { Anzahl der Patienten } \\
\text { insgesamt }\end{array}$ & $\begin{array}{c}\text { \% der } \\
\text { Erstkontakte }\end{array}$ \\
\hline oberer Atemwegsinfekt & 90 & 48,4 \\
akute Bronchitis & 46 & 24,7 \\
akute Tonsillitis & 21 & 11,3 \\
akute Sinusitis & 19 & 10,2 \\
akute Exazerbationen COPD & 12 & 6,5 \\
akute Laryngitis & 8 & 4,3 \\
akute Otitis media & 4 & 2,2 \\
Tubenkatarrh & 4 & 2,2 \\
\hline
\end{tabular}

\subsubsection{Bei Folgekontakten}

Eine Aufstellung der insgesamt gestellten Diagnosen bezüglich der Erkrankungen der Atemwege und des Ohres bei Folgekontakten findet sich in Tabelle 8. Da Mehrfachdiagnosen möglich waren, ergab die Summe der Prozentangaben mehr als 100\%. Auch bei den Folgekontakten überwogen die akuten Erkrankungen der Atemwege. Im Vergleich zu den Erstkontakten zeigte sich jedoch bei Folgekontakten ein signifikant höherer Anteil von akuten Exazerbationen chronisch obstruktiver Lungenerkrankungen $(6,5 \%$ gegen $13,8 \%, \mathrm{p}=0,046)$.

Tabelle 8: Häufigkeit gestellter Diagnosen in Erst- oder Zweitdiagnose bei Folgekontakten

\begin{tabular}{lcc}
\hline \multicolumn{1}{c}{ Diagnose nach ICPC } & $\begin{array}{c}\text { Anzahl der Patienten } \\
\text { insgesamt }\end{array}$ & $\begin{array}{c}\text { \% der } \\
\text { Folgekontakte }\end{array}$ \\
\hline oberer Atemwegsinfekt & 27 & 31,0 \\
akute Bronchitis & 24 & 27,6 \\
akute Sinusitis & 14 & 16,1 \\
akute Exazerbationen COPD & 12 & 13,8 \\
akute Tonsillitis & 9 & 10,3 \\
akute Otitis media & 6 & 6,9 \\
akute Laryngitis & 4 & 4,6 \\
Tubenkatarrh & 2 & 2,3 \\
Pneumonie & 1 & 1,2 \\
Maligne Tumoren Trachea/Bronchien/Lunge & 1 & 1,2 \\
\hline
\end{tabular}




\subsection{Therapie}

\subsubsection{Anzahl der verordneten Medikamente pro Patient}

In Tabelle 9 wird die Anzahl der verordneten Medikamente bei Erst- und Folgekontakten wiedergegeben. Es wurden Verordnungen auf Kassenrezept, auf Privatrezept und die Mitgabe von Arzneimittelmustern berücksichtigt. Allen Patienten, die ein Musterpräparat erhielten, wurden noch zusätzlich ein oder mehrere Medikamente auf Privat- oder Kassenrezept verordnet. Die Empfehlung zum Selbstkauf von Medikamenten wird in diesem Kapitel nicht berücksichtigt und in Kapitel 3.6.6 gesondert dargestellt.

98,4\% der Erstkontakte erhielten ein Rezept und/oder ein Musterpräparat. Bei den Folgekontakten erhielten 65,5\% der Patienten eine Verordnung eines Medikamentes, das sie während dieses Krankheitsereignisses zuvor noch nicht eingenommen hatten. Bei 33,3\% der Patienten wurde die initial begonnene Therapie beibehalten und kein weiteres Medikament verordnet. Bei einem Patienten wurde die Therapie komplett abgesetzt.

Bei der Erstkonsultation verschrieben die Ärzte durchschnittlich 2,1 $( \pm 1,0)$ Medikamente, bei Folgekonsultationen 1,3 $( \pm 1,1)$ Medikamente. Bei Folgekontakten unterschied sich die Zahl der verordneten Medikamente signifikant $(\mathrm{t}=4,61, \mathrm{p}<0,001, \mathrm{df}=85)$ von denen der Erstkontakte. Kassenpatienten erhielten durchschnittlich 1,8 $( \pm 1,1)$ Medikamente, Privatpatienten 2,6 $( \pm 0,9)$. Dabei bekamen Privatpatienten bei Erstkontakten signifikant mehr Medikamente als Kassenpatienten $(\mathrm{t}=2,15, \mathrm{p}=0,017, \mathrm{df}=184)$. Dieser Unterschied war bei Folgekontakten nicht signifikant $(\mathrm{t}=0,865, \mathrm{p}=0,195, \mathrm{df}=85)$. 
Tabelle 9: Anzahl der verordneten Medikamente pro Patient (Anzahl der Patienten absolut und in \%)

\begin{tabular}{lcrr}
\hline \multicolumn{1}{c}{$\begin{array}{c}\text { Anzahl der verordneten } \\
\text { Medikamente }\end{array}$} & $\begin{array}{c}\text { Patientenanzahl } \\
\text { insgesamt }\end{array}$ & Erstkontakte & Folgekontakte \\
\hline kein Medikament & $33(12,4 \%)$ & $3(1,6 \%)$ & $30(37,0 \%)$ \\
1 Medikament & $79(29,6 \%)$ & $58(31,2 \%)$ & $21(25,9 \%)$ \\
2 Medikamente & $88(33,0 \%)$ & $63(33,9 \%)$ & $25(30,9 \%)$ \\
3 Medikamente & $55(20,6 \%)$ & $48(25,8 \%)$ & $7(8,6 \%)$ \\
4 Medikamente & $13(4,9 \%)$ & $11(5,9 \%)$ & $2(2,5 \%)$ \\
5 Medikamente & $5(1,9 \%)$ & $3(1,6 \%)$ & $2(2,5 \%)$ \\
\hline
\end{tabular}

\subsubsection{Medikamentöse Therapie}

In Tabelle 10 wird eine Unterteilung der medikamentösen Therapie in Verordnungen bei Patienten mit Erst- oder Folgekontakten gemäß ATC-Code vorgenommen. Es werden bei den Folgekontakten nur die neu verordneten Medikamente und nicht die Beibehaltung einer bereits begonnenen Therapie nach dem Erstkontakt berücksichtigt. Die am häufigsten verordnete Hauptgruppe der Husten- und Erkältungspräparate wird in Tabelle 11 weiter differenziert.

Tabelle 10: Medikamentöse Therapie

\begin{tabular}{lcc}
\hline \multicolumn{1}{c}{ Therapeutische Hauptgruppe } & Erstkontakte in \% & Folgekontakte in \% \\
\hline Husten- und Erkältungspräparate & 87,1 & 52,9 \\
Systemische Antibiotika & 43,5 & 29,9 \\
Rhinologika & 17,7 & 11,5 \\
Antiasthmatika & 7,5 & 8,1 \\
Halsschmerzmittel & 4,3 & 2,3 \\
Brusteinreibungen und sonstige Inhalate & 3,2 & 3,5 \\
Echinacea-Zubereitungen & 2,2 & 0,0 \\
Otologika & 2,2 & 1,2 \\
\hline
\end{tabular}


Tabelle 11: Anzahl der Verordnungen innerhalb der pharmakologischen Hauptgruppen der Husten- und Erkältungspräparate

\begin{tabular}{lcc}
\hline \multicolumn{1}{c}{ Pharmakologische Hauptgruppe } & $\begin{array}{c}\text { Erstkontakte } \\
\text { in \% }\end{array}$ & $\begin{array}{c}\text { Folgekontakte } \\
\text { in \% }\end{array}$ \\
\hline Expektoranzien exkl. Kombinationen mit Antitussiva & 67,2 & 43,7 \\
Andere Erkältungspräparate (z.B. analgetikahaltige & 26,3 & 9,2 \\
Grippemittel) & & \\
Antitussiva exkl. Kombinationen mit Expektoranzien & 16,1 & 12,6 \\
$\begin{array}{l}\text { Antitussiva- und Expektoranzien-Kombinationen mit } \\
\text { Antibiotika }\end{array}$ & 6,5 & 2,3 \\
$\begin{array}{l}\text { Erkältungspräparate exkl. Kombinationen mit Antiinfektiva } \\
\text { (z.B. Kombinationen verschiedener Homöopathika) }\end{array}$ & 4,3 & 0,0 \\
\hline
\end{tabular}

Bei Betrachtung der chemischen Substanzen der einzelnen pharmakologischen Hauptgruppen befanden sich die insgesamt am häufigsten verordneten Medikamente in der Hauptgruppe der Husten- und Erkältungspräparate. Die meisten Verordnungen entfielen auf das Acetylcystein aus der Gruppe der Expektoranzien (32,6\%). 15,8\% der Patienten erhielten Ambroxol, darunter 14mal als Kombinationspräparat, bevorzugt in der Kombination mit Doxycyclin. Ebenfalls 15,8\% der Patienten wurde Paracetamol verschrieben, wobei zu berücksichtigen ist, dass 6 Patienten empfohlen wurde sich das Präparat selbst zu kaufen.

\subsubsection{Verordnung von pflanzlichen Arzneien und Homöopathika}

Der Anteil der pflanzlichen Arzneien an der Gesamtzahl der Verordnungen betrug 12,9\%. Es wurden Präparate aus den therapeutischen Hauptgruppen der Halsschmerzmittel, Brusteinreibungen und sonstigen Inhalate, Husten- und Erkältungspräparate und EchinaceaZubereitungen gewählt. Auch hier gehörte der größte Teil zu der Gruppe der Husten- und Erkältungspräparate. Die Kombinationen mehrerer pflanzlicher Expektoranzien aus dieser Gruppe standen mit 27 Verordnungen an 4. Stelle der insgesamt am häufigsten verordneten Medikamente. Homöopathika machten in Bezug auf die Gesamtverordnungen einen Anteil von 2,2\% aus. Dabei wurden ausschließlich Kombinationen verschiedener Homöopathika (sogenannte Komplexpräparate) verschrieben. 


\subsubsection{Arzneiwünsche der Patienten}

Nur 8 Patienten baten den Arzt um die Verschreibung eines bestimmten Präparates (2,9\%). Diese wünschten jeweils die Verordnung eines Medikamentes. 4 erhielten das von ihnen gewünschte Präparat. Ein Patient bat um die Verschreibung eines Antibiotikums; dieser Bitte wurde entsprochen. Bei 4 weiteren Patienten mit einem Arzneiwunsch wurde zusätzlich ein Antibiotikum verordnet. 2-mal wurde die Verordnung von Acetylcystein gewünscht und einmal daraufhin verordnet. 2 Personen baten um Nasentropfen, in einem Fall wurde dem Anliegen entsprochen. Jeweils einmal wünschten die Patienten eine Verordnung einer Brusteinreibung, einer Acetylcystein-Kombination oder einer Kombination mehrerer pflanzlicher Expektoranzien. Die Patientenwünsche hielten sich insgesamt jedoch in relativ engen Grenzen.

\subsubsection{Mitgabe von Arzneimitteln als Musterpräparat}

Es wurden 17 Arzneimittel als Musterpräparate an insgesamt 14 Patienten weitergegeben. 11 Patienten erhielten ein solches, 3 Patienten wurden 2 Musterpräparate mitgegeben. Tabelle 12 zeigt eine Übersicht der ausgehändigten Musterpräparate. In der Gruppe der Husten- und Erkältungspräparate wurde 5-mal Acetylcystein als Musterpräparat an Patienten weitergeben.

Tabelle 12: Art der mitgegebenen Musterpräparate

\begin{tabular}{lc}
\hline \multicolumn{1}{c}{ Therapeutische Hauptgruppe } & Mitgabe als Musterpräparat \\
\hline Husten- und Erkältungspräparate & 8 \\
Antibiotika & 4 \\
Antiasthmatika & 2 \\
Rhinologika & 1 \\
Brusteinreibungen und sonstige Inhalate & 1 \\
Analgetika & 1 \\
\hline
\end{tabular}




\subsubsection{Empfehlungen zum Selbstkauf von Medikamenten}

7 Patienten wurden Medikamente zum Selbstkauf empfohlen (2,6\%). Bei 5 Patienten erfolgte der gezielte Hinweis auf ein Medikament, bei 2 Patienten bezog es sich auf 2 Präparate. Am häufigsten wurde den erkrankten Personen der Selbstkauf von Paracetamol nahegelegt (5 Empfehlungen); 2-mal ging die Empfehlung in Richtung xylometazolinhaltige Nasentropfen und einmal sollten Kamillenblüten-Kombinationen als Rachentherapeutika angewendet werden.

\subsubsection{Ausstellung einer Arbeitsunfähigkeitsbescheinigung (AU)}

Von den insgesamt 132 erwerbstätigen Patienten (48,4\% aller Patienten) erhielten 75 Patienten (56,8\%) eine Arbeitsunfähigkeitsbescheinigung, 25,0\% erhielten keine Krankschreibung und 18,2\% lehnten sie ab. Die Dauer der Erstbescheinigung betrug bei Erst- bzw. Folgekontakten im Durchschnitt 4,2 $( \pm 1,6)$ Tage. Folgebescheinigungen wurden im Mittelwert über 4,1 $( \pm 1,6)$ Tage ausgestellt. Bei den Erstkontakten erhielten 55,4\% der Erwerbstätigen eine derartige Bescheinigung. Bei den Folgekontakten wurden 60,0\% der erwerbstätigen Patienten krank geschrieben, wobei 55,0\% eine erneute Bescheinigung der Arbeitsunfähigkeit und 5,0\% eine Erstbescheinigung erhielten.

\subsubsection{Wiedereinbestellung von Patienten}

116 Patienten (42,5\%) wurden gebeten sich erneut beim Arzt vorzustellen. Bei 35,5\% hielten die Ärzte eine Wiedervorstellung für nicht erforderlich. Die restlichen $22,0 \%$ erhielten die Anweisung bei einer Verschlechterung des Zustandes wieder zu kommen. Bei Betrachtung der 4 am häufigsten gestellten Diagnosen wurde 63,3\% an einer akuten Tonsillitis, 61,4\% an einer akuten Bronchitis, 48,5\% an einer akuten Sinusitis und 23,1\% an einem oberen Atemwegsinfekt Erkrankten eine Wiedervorstellung nahegelegt. 


\subsubsection{Empfehlungen zur Selbstmedikation und nichtmedikamentösen Therapie}

Von den 41 Personen, die eine Selbstmedikation durchgeführt hatten, erhielten 16 Patienten $(39,0 \%)$ eine Verordnung aus derselben therapeutischen Hauptgruppe (Husten- und Erkältungspräparate, Rhinologika, Echinacea-Zubereitungen). 11 Patienten wurde die selbe chemische Substanz $(26,8 \%)$ verschrieben. Husten- und Erkältungspräparate wurden erneut am häufigsten verordnet, Halsschmerzmittel wurden nicht eingesetzt.

34,8 \% der Patienten erhielten zusätzlich zur medikamentösen Therapie Empfehlungen zur nichtmedikamentösen Behandlung. Tabelle 13 gibt eine Übersicht der zusätzlichen Empfehlungen. Zur Inhalation sollten Kamille, Kochsalz, ätherische Öle und Salbei benutzt werden. In 2 Praxen waren Geräte zur Bestrahlung vorhanden; so wurde 2-mal eine Mikrowellenbestrahlung und einmal eine Kurzwellenbestrahlung angeordnet. 3 weitere Patienten erhielten die Empfehlung zu Hause eine Rotlichtbestrahlung durchzuführen. In einer Praxis hatten die mitarbeitenden Ärzte ein Informationsblatt zur unterstützenden Therapie und zusätzlichen Informationen zur Selbstmedikation für die Patienten angefertigt. Allerdings wurde während der Hospitation keinem dieses Informationsblatt ausgehändigt.

Tabelle 13: Empfehlungen zur nichtmedikamentösen Therapie

\begin{tabular}{lccc}
\hline & Patienten insgesamt & Erstkontakte & Folgekontakte \\
\hline erhöhte Flüssigkeitszufuhr & 52 & 39 & 13 \\
Inhalation & 48 & 36 & 12 \\
Rauchverbot & 13 & 13 & 0 \\
Schonung & 12 & 10 & 2 \\
Bettruhe & 11 & 10 & 1 \\
Bestrahlung & 6 & 4 & 2 \\
Gurgeln mit Kamille/Salbei & 5 & 4 & 1 \\
Halswickel & 2 & 1 & 1 \\
Wadenwickel & 1 & 1 & 0 \\
frische Luft & 1 & 1 & 0 \\
Akupressur der NNH & 1 & 0 & 1 \\
Brusteinreibungen & 1 & 1 & 0 \\
Vitaminzufuhr & 1 & 1 & 0 \\
\hline
\end{tabular}




\subsubsection{Zusätzliche Empfehlungen im Bezug auf die Verordnung von Acetylcystein}

Wie bereits in Kapitel 3.6.2 ausgeführt, wurde Acetylcystein mit 89-mal und damit am häufigsten verordnet. Es ist u.a. aus dem Beipackzettel der Firmen bekannt, dass dessen schleimlösende Wirkung durch zusätzliche Flüssigkeitszufuhr unterstützt wird. 27,0\% der Patienten, die ein Medikament mit dem Wirkstoff Acetylcystein verordnet bekamen, erhielten die entsprechende Empfehlung einer erhöhten Flüssigkeitsaufnahme.

\subsubsection{Raucheranamnese und Erteilung eines Rauchverbotes}

86 Patienten wurden vom Arzt gefragt, ob sie rauchen (31,5\%). Dabei gaben 48 an, Raucher zu sein. Im Durchschnitt konsumierten sie 15,9 $( \pm 7,9)$ Zigaretten täglich. 27,1\% dieser Patienten wurde vom Arzt empfohlen das Rauchen einzustellen.

\subsection{Verordnung von Antibiotika}

\subsubsection{Unterscheidung der Antibiotika nach ihrer chemischen Substanz}

Von den 273 beobachteten Patienten erhielten 133 (48,7\%) beim Erst- oder Folgekontakt ein Antibiotikum. 43,6\% der Erstkontakte und 29,9\% der Folgekontakte wurde während der Hospitation ein Antibiotikum verordnet. Von den 87 Patienten mit einer Folgekonsultation hatten bereits 37 bei der Erstkonsultation ein Antibiotikum (42,5\%) erhalten. Beim Folgekontakt verordneten die Ärzte bei 10 dieser 37 Patienten erneut ein Antibiotikum; 5 Patienten erhielten das gleiche Präparat, bei 5 anderen wurde es gewechselt. Bei 15 Patienten (17,2\%), die initial kein Antibiotikum verordnet bekommen hatten, erschien den Ärzten beim Folgekontakt ein Antibiotikum indiziert. Somit sollten 52 der 87 Patienten (59,8\%) mit Folgekontakt ein Antibiotikum nach Erst- oder Folgekontakt einnehmen. 
In Tabelle 14 wird die Gesamtverordnung von Antibiotika bei Atemwegsinfektionen dargestellt. Es werden auch Antibiotika berücksichtigt, die Patienten mit Folgekonsultation bei ihrer Erstkonsultation initial verordnet bekamen.

Tabelle 14: Verordnete Antibiotika (Gesamtverordnungen und prozentualer Anteil an den Antibiotikaverordnungen insgesamt)

\begin{tabular}{|c|c|c|c|}
\hline $\begin{array}{c}\text { Pharmakologische } \\
\text { Hauptgruppe }\end{array}$ & $\begin{array}{l}\text { Chemische } \\
\text { Substanz }\end{array}$ & $\begin{array}{l}\text { insgesamt } \\
\text { verordnet }\end{array}$ & $\begin{array}{c}\text { prozentualer } \\
\text { Anteil }\end{array}$ \\
\hline \multirow{6}{*}{$\begin{array}{l}\text { Antitussiva-Expektoranzien- } \\
\text { Kombinationen mit Antibiotika }\end{array}$} & Doxycyclin- & 21 & 14,6 \\
\hline & Kombinationen & & \\
\hline & Tetracyclin- & 3 & 2,1 \\
\hline & Kombinationen & & \\
\hline & Cefaclor- & 1 & 0,7 \\
\hline & Kombinationen & & \\
\hline \multirow{7}{*}{$\begin{array}{l}\text { Tetracycline } \\
\text { Betalactamantibiotika/Penicilline }\end{array}$} & Doxycyclin & 9 & 6,3 \\
\hline & Ampicillin & 1 & 0,7 \\
\hline & Amoxicillin & 15 & 10,4 \\
\hline & $\begin{array}{l}\text { Phenoxymethyl- } \\
\text { penicillin }\end{array}$ & 12 & 1,4 \\
\hline & Propicillin & 1 & 0,7 \\
\hline & Benzylpenicillin- & 2 & 1,4 \\
\hline & Kombinationen & & \\
\hline \multirow[t]{6}{*}{ andere Betalactamantibiotika } & Cefaclor & 7 & 4,9 \\
\hline & Cefadroxil & 1 & 0,7 \\
\hline & Cefixim & 2 & 1,4 \\
\hline & Cefpodoxim & 1 & 0,7 \\
\hline & Cefuroximaxetil & 8 & 5,6 \\
\hline & Loracarbef & 2 & 1,4 \\
\hline \multirow[t]{5}{*}{ Makrolide und Lincosamide } & Erythromycin & 6 & 4,2 \\
\hline & Roxithromycin & 24 & 16,7 \\
\hline & Clarithromycin & 4 & 2,8 \\
\hline & Azithromycin & 19 & 13,2 \\
\hline & Clindamycin & 1 & 0,7 \\
\hline \multirow[t]{2}{*}{ Chinolonantibiotika } & Ciprofloxacin & 3 & 2,1 \\
\hline & Pefloxacin & 1 & 0,7 \\
\hline
\end{tabular}

Die pharmakologische Hauptgruppe der Makrolide und Lincosamide wurde insgesamt am häufigsten verordnet $(37,5 \%)$, an zweiter Stelle folgten Betalactamantibiotika und Penicilline (21,5\%). Doxycyclin als Monosubstanz oder in Kombination mit Expektoranzien (20,8\%) nahm 
den 3. Platz ein. Ein geringerer Anteil entfiel auf die Gruppe der anderen Betalactamantibiotika $(14,6 \%)$. Die Verordnung von Chinolonen stellte eher eine Ausnahme dar.

\subsubsection{Diagnosebezogene Antibiotikaverordnung}

Bei Erstkontakten mit der Diagnose einer akuten Tonsillitis oder akuten Bronchitis wurde bei über $80 \%$ der Patienten der Einsatz eines Antibiotikums für sinnvoll erachtet. Die meisten Antibiotikaverordnungen fanden sich bei der Diagnose der akuten Tonsillitis (85,7\%). Den geringsten Anteil an diesen Verordnungen nahm der obere Atemwegsinfekt $(15,6 \%)$ ein (s. Tabelle 15). Bei Folgekontakten war es anhand der ärztlichen Dokumentation möglich auch die Verschreibung von Antibiotika beim Erstkontakt zu berücksichtigen. Folgekontakte führten bei fast allen Krankheitsbildern mit Ausnahme der akuten Bronchitis und des akut exazerbierten Lungenemphysems sowie des akut exazerbierten Asthma bronchiale zu einem Anstieg der Antibiotikaverordnungen. Alle Patienten mit der Diagnose einer akuten Tonsillitis oder Laryngitis hatten beim Folgekontakt ein Antibiotikum erhalten.

Tabelle 15: Diagnosebezogene Verordnung von Antibiotika ( $\mathrm{n}=$ Anzahl der Patienten mit dieser Diagnose, mehrere Diagnosen pro Patient möglich)

\begin{tabular}{lcccc}
\hline \multicolumn{1}{c}{$\begin{array}{c}\text { Diagnosen nach } \\
\text { ICPC }\end{array}$} & $\begin{array}{c}\text { n } \\
\text { Erstkontakte }\end{array}$ & $\begin{array}{c}\text { Erstkontakte } \\
\text { mit Antibiotika- } \\
\text { verordnung }\end{array}$ & $\begin{array}{c}\text { n } \\
\text { Folgekontakte }\end{array}$ & $\begin{array}{c}\text { Folgekontakte } \\
\text { mit Antibiotika- } \\
\text { verordnung }\end{array}$ \\
\hline oberer & 90 & $15,6 \%$ & 27 & $25,9 \%$ \\
Atemwegsinfekt & & & & \\
akute Sinusitis & 19 & $57,9 \%$ & 14 & $62,3 \%$ \\
akute Tonsillitis & 21 & $85,7 \%$ & 9 & $100,0 \%$ \\
akute Laryngitis & 8 & $62,5 \%$ & 4 & $100,0 \%$ \\
akute Bronchitis & 46 & $80,4 \%$ & 24 & $70,8 \%$ \\
akute Exazerbationen & 12 & $41,7 \%$ & 12 & $66,7 \%$ \\
COPD & & & & \\
\hline
\end{tabular}

Bei der Untersuchung des Zusammenhanges zwischen Antibiotikatherapie und gestellter Diagnose mittels multipler logistischer Regressionsanalyse zeigte sich, dass Antibiotika vermehrt bei akuter Tonsillitis eingesetzt wurden (OR 2,68, 95\%-CI: 1,07-6,73). Demgegenüber wurden sie signifikant seltener bei der Diagnose oberer Atemwegsinfekt verordnet (OR 0,14, 95\%-CI 
0,07-0,25). Alle anderen Diagnosen wiesen keine signifikante Assoziation auf (akute Bronchitis, akute Sinusitis, akute Laryngitis, akute Exazerbationen chronisch obstruktiver Lungenerkrankungen, akute Otitis media).

\subsubsection{Einfluss von Symptomen und Untersuchungsbefunden auf die Antibiotikaverordnung}

Die folgende Darstellung möglicher Einflussfaktoren auf die Antibiotikaverordnung beschränkt sich auf die Erstkontakte, da die ärztliche Dokumentation von Anamnese und Untersuchungsbefunden bei den initialen Konsultationen der Folgekontakte nicht oder nur teilweise vorlag. Da lediglich 12 Patienten zusätzlich eine Antibiotikaverordnung erhielten, denen initial beim Erstkontakt kein Antibiotikum verordnet worden war, ist hier nur von einer geringen Beeinflussung auszugehen.

Bei den im Weiteren dargestellten Einflussfaktoren konnte nur der Kreis der Patienten ausgewertet werden, die dieses Symptom äußerten bzw. von dem Arzt diesbezüglich befragt oder bei denen bestimmte Untersuchungsbefunde erhoben wurden. Im Chi-Quadrat-Test wurde das entsprechende Symptom bzw. der Untersuchungsbefund jeweils mit der Situation, dass es bzw. er nicht vorlag, verglichen inklusive der hierzu nicht befragten Patienten.

Tabelle 16: Einfluss der geschilderten Symptome auf die Verordnung von Antibiotika (signifikante Werte fett hervorgehoben, n.s. = nicht signifikant)

\begin{tabular}{lccc}
\hline \multicolumn{1}{c}{ Symptome } & $\begin{array}{c}\text { Anzahl der } \\
\text { Patienten }\end{array}$ & $\begin{array}{c}\text { Patienten mit } \\
\text { Antibiotikaverordnung }\end{array}$ & Chi-Quadrat-Test \\
\hline Husten & 146 & $43,8 \%$ & n.s. \\
Schnupfen & 105 & $38,1 \%$ & n.s. \\
Halsschmerzen & 73 & $46,6 \%$ & n.s. \\
Fieber & 61 & $42,3 \%$ & n.s. \\
Kopfschmerzen & 57 & $47,4 \%$ & n.s. \\
Abgeschlagenheit & 42 & $57,1 \%$ & n.s. \\
Heiserkeit & 31 & $19,4 \%$ & $\mathbf{p}<\mathbf{0 , 0 1}$ \\
Gliederschmerzen & 30 & $53,3 \%$ & n.s. \\
Ohrenschmerzen & 24 & $45,8 \%$ & n.s. \\
Schmerzen NNH & 14 & $50,0 \%$ & n.s. \\
\hline
\end{tabular}


Die Schilderung des Symptoms Husten führte zu keiner signifikant erhöhten Antibiotikagabe im Vergleich zum Nichtauftreten dieses Symptoms (Tabelle 16). Um zu erfassen, inwieweit die weitere Differenzierung des Symptoms Husten einen Einfluss auf das Verordnungsverhalten der Ärzte hatte, erfolgten weitere Berechnungen. Demnach führte das Auftreten von produktivem feuchtem Husten im Vergleich zum Erscheinungsbild eines unproduktiven trockenen Hustens zu keiner signifikant erhöhten Verordnung von Antibiotika. Wenn der Patient jedoch Husten mit gelblich-grünem Auswurf schilderte, wurde signifikant häufiger ein Antibiotikum verschrieben als bei Patienten mit weißlichem Auswurf $(p<0,01)$.

Bei der Auskultation der Lunge wurde zwischen einer Auskultation von pathologischen Atemgeräuschen und einer von pathologischen Nebengeräuschen unterschieden. Es zeigte sich, dass im Vergleich zum normalen Befund die Auskultation von pathologischen Atemgeräuschen (53,9\% der Patienten mit Antibiotikaverordnung) zu keiner signifikant erhöhten Antibiotikagabe führte. Die Auskultation pathologischer Nebengeräuschen $(69,6 \%$ der Patienten mit Antibiotikaverordnung) war jedoch signifikant $(\mathrm{p}<0,0001)$. Das Vorhandensein von Stippchen oder Belägen auf den Tonsillen (87,5\% der Patienten mit Antibiotikaverordnung) führte im Vergleich zum normalen Befund zu einer signifikant erhöhten Antibiotikagabe $(\mathrm{p}<0,05)$. Lediglich gerötete Tonsillen (44,8\% der Patienten mit Antibiotikaverordnung) führten jedoch zu keiner erhöhten Verschreibung von Antibiotika. Allerdings erhielten 4 Patienten mit lediglich geröteten Tonsillen und der Diagnose einer akuten Tonsillitis ein Antibiotikum, ohne dass Tonsillen mit Belägen oder Stippchen vorlagen.

Ältere (ab 60 Jahren) erhielten nicht signifikant häufiger ein Antibiotikum als Jüngere (unter 60 Jahren). Kein signifikanter Unterschied der Antibiotikaverordnung ergab sich bezüglich der Erwerbstätigkeit der Behandelten im Vergleich zu Rentnern, Schülern und Studenten und NichtErwerbstätigen.

Ein positiver Zusammenhang zwischen Antibiotikaverordnung und Wiedereinbestellung des Patienten konnte nachgewiesen werden. Diejenigen, denen eine Wiedervorstellung empfohlen wurde (71,8\% der Patienten mit Antibiotikaverordnung), erhielten signifikant häufiger ein Rezept für ein Antibiotikum $(\mathrm{p}<0,0001)$. Dabei bestand dieser Zusammenhang sowohl für alle wieder 
einbestellten Patienten als auch für jene mit der Diagnose des oberen Atemwegsinfektes oder der akuten Bronchitis. Patienten, denen keine Wiedervorstellung $(25,0 \%$ der Patienten mit Antibiotikaverordnung) oder nur beim Auftreten von Beschwerden (20,8\% der Patienten mit Antibiotikaverordnung) nahegelegt wurde, erhielten nicht signifikant häufiger ein Antibiotikum.

Zur weiteren Analyse relevanter Befunde wurde ergänzend eine multiple logistische Regressionsanalyse durchgeführt (Tabelle 17). Antibiotika wurden gehäuft bei auffälligen körperlichen Untersuchungsbefunden verordnet. Demnach wiesen vor allem mit Stippchen belegten Tonsillen eine starke Assoziation zur Verschreibung von Antibiotika auf. Aber auch das Symptom gelblicher Auswurf war mit einer vermehrten Verordnung von Antibiotika verbunden. Folgende Variablen waren ohne Einfluss: Alter, Raucherstatus, schmerzende Nasennebenhöhlen, gerötete Tonsillen, Husten, Halsschmerzen, Auswurf, weißliches Sputum, Heiserkeit, Schnupfen, Ohrenschmerzen und Kopfschmerzen.

Tabelle 17: Abhängigkeit der Antibiotikatherapie von Symptomen und Untersuchungsbefunden mittels multipler logistischer Regressionsanalyse

\begin{tabular}{lcc}
\hline \multicolumn{1}{c}{ Symptom/Befund } & OR & 95\%-CI \\
\hline Tonsillen mit Stippchen bei der Inspektion des Nasen-Rachen- & 15,41 & $3,6-66,16$ \\
Raumes & & \\
pathologischer Otoskopiebefund & 8,85 & $1,16-67,58$ \\
pathologischer Palpationsbefund der Halslymphknoten & 6,24 & $1,97-19,71$ \\
Auskultation pathologischer Nebengeräusche & 4,29 & $2,09-8,83$ \\
pathologischer Palpationsbefund der Nasennebenhöhlen & 3,20 & $1,38-7,42$ \\
Abgeschlagenheit & 3,09 & $1,42-6,72$ \\
Auskultation pathologischer Atemgeräusche & 2,91 & $1,17-7,23$ \\
Fieber & 2,19 & $1,06-4,54$ \\
gelbes Sputum & 2,10 & $1,07-4,14$ \\
\hline
\end{tabular}

\subsubsection{Praxischarakteristika als Einflussfaktoren auf die Antibiotikaverordnung}

Im Folgenden wurden weitere mögliche Einflussfaktoren von Seiten der behandelnden Ärzte untersucht. Bezogen auf die Häufigkeit von Antibiotikaverordnungen bestand zwischen Ärzten aus Brilon und Hildesheim kein signifikanter Unterschied. Die teilnehmenden Ärzte in Brilon verschrieben in 43,8\% der Fälle ein Antibiotikum, in Hildesheim betrug der Anteil 42,5\%. 
Die Häufigkeit derartiger Verschreibungen war abhängig vom Alter des verordnenden Arztes. Bei denen, die jünger als 50 Jahre waren, erhielten 37,3\% der Patienten ein Antibiotikum gegenüber $54,4 \%$ bei Ärzten mit einem Lebensalter höher oder gleich 50 Jahre $(\mathrm{p}<0,05)$. Dabei ist die gestiegene Rate an Antibiotikaverordnungen nicht auf die höhere Altersstruktur der Patienten bei Ärzten über 50 Jahren zurückzuführen, da sich diese nicht signifikant unterschied. Die Niederlassungsdauer stellte ebenfalls keinen signifikanten Einflussparameter auf die Antibiotikaverordnung dar.

Ärzte aus Gemeinschaftspraxen verordneten signifikant weniger Antibiotika als Einzelpraxisinhaber (34,0\% der Patienten gegenüber 53,3\% in Einzelpraxen, $\mathrm{p}<0,01)$. Auch hier findet sich kein signifikanter Unterschied in der Alterszugehörigkeit der Patienten bei Einzelpraxen im Vergleich zu Gemeinschaftspraxen.

Die Praxisgröße, gemessen an der abgerechneten Scheinzahl pro Quartal $(<1500$ und $>1500$ Scheine pro Quartal), stellte keinen signifikanten Einflussparameter, der bei der Verordnung von Antibiotika zu berücksichtigen wäre, dar.

\subsection{Verordnung von Husten- und Erkältungspräparaten}

\subsubsection{Diagnosebezogene Verordnung von Husten- und Erkältungspräparaten}

Wie in Kapitel 3.6.2 dargestellt bezogen sich die meisten Verordnungen auf Präparate aus der Gruppe der Husten- und Erkältungspräparate. 87,1\% der Erstkontakte und 52,9\% der Folgekontakte erhielten eine Verordnung aus dieser Gruppe. In Tabelle 18 wird die Häufigkeit der diagnosebezogenen Verschreibung dieser Medikamente bei Erst- und Folgekontakten dargestellt. Mit Ausnahme der Diagnose der akuten Tonsillitis lag die Rate bei Erstkontakten in der Größenordnung von $90 \%$ und darüber. Bei Folgekontakten war es anhand der ärztlichen Dokumentation möglich auch die initiale Verordnung von Husten- und Erkältungspräparaten beim Erstkontakt zu berücksichtigen. 
Tabelle 18: Diagnosebezogene Verordnung von Husten- und Erkältungspräparaten (n=Anzahl der Patienten mit dieser Diagnose, mehrere Diagnosen pro Patient möglich)

\begin{tabular}{lcccc}
\hline Diagnose nach ICPC & $\begin{array}{c}\text { n } \\
\text { Erst- } \\
\text { kontakte }\end{array}$ & $\begin{array}{c}\text { Erstkontakte mit } \\
\text { Verordnung von } \\
\text { Husten- und } \\
\text { Erkältungs- } \\
\text { präparaten }\end{array}$ & $\begin{array}{c}\text { n } \\
\text { Folge- } \\
\text { kontakte }\end{array}$ & $\begin{array}{c}\text { Folgekontakte mit } \\
\text { Verordnung von } \\
\text { Husten- und } \\
\text { Erkältungs- } \\
\text { präparaten }\end{array}$ \\
\hline oberer & 90 & $90,0 \%$ & 27 & $100,0 \%$ \\
Atemwegsinfekt & 46 & $93,5 \%$ & 24 & $100,0 \%$ \\
akute Bronchitis & 21 & $57,1 \%$ & 9 & $77,8 \%$ \\
akute Tonsillitis & 19 & $94,7 \%$ & 14 & $100,0 \%$ \\
akute Sinusitis & 8 & $88,9 \%$ & 4 & $100,0 \%$ \\
akute Laryngitis & 12 & $100,0 \%$ & 12 & $91,7 \%$ \\
akute Exazerbationen & & & & \\
COPD & & & & \\
\hline
\end{tabular}

In einer ergänzend durchgeführten logistischen Regressionsanalyse war die Verordnung von Husten- und Erkältungspräparaten nur mit der Diagnose der akuten Tonsillitis negativ assoziiert. Demnach wurden diese Präparate bei der akuten Tonsillitis weniger häufig verordnet (OR 0,25, 95\%-CI: 0,11-0,55). Alle anderen Diagnosen wiesen keine Assoziation auf (oberer Atemwegsinfekt, akute Bronchitis, akute Sinusitis, akute Exazerbationen chronisch obstruktiver Lungenerkrankungen, akute Otitis media).

\subsubsection{Einfluss von Symptomen und Untersuchungsbefunden auf die Verordnung von Husten- und Erkältungspräparaten}

In Tabelle 19 werden die von den Patienten geschilderten oder von den Ärzten anamnestisch erhobenen Symptome und Untersuchungsbefunde zusammen mit der Häufigkeit von Verordnungen aus der Gruppe der Husten- oder Erkältungspräparate dargestellt. Die Ergebnisse möglicher Einflussfaktoren beziehen sich auf Erstkontakte, weil bei diesen Patienten eine lückenlose Dokumentation hinsichtlich Diagnostik und Therapie vorlag. Im Chi-Quadrat-Test wurde das entsprechende Symptom bzw. der Untersuchungsbefund jeweils mit dem Nichtvorliegen verglichen inklusive der hierzu nicht befragten Patienten. Sämtliche Symptome wiesen dabei einen sehr hohen Anteil an Verordnungen dieser Gruppe auf. 
Tabelle 19: Einfluss geschilderter Symptome auf die Verordnung von Husten- und Erkältungspräparaten (signifikante Werte fett hervorgehoben, n.s.= nicht signifikant)

\begin{tabular}{lccc}
\hline \multicolumn{1}{c}{ Symptome } & $\begin{array}{c}\text { Anzahl } \\
\text { der } \\
\text { Patienten }\end{array}$ & $\begin{array}{c}\text { Patienten mit Verordnungen von } \\
\text { Husten- und Erkältungspräparaten }\end{array}$ & Chi-Quadrat-Test \\
\hline Husten & 146 & $91,8 \%$ & $\mathbf{p}<\mathbf{0 , 0 0 0 1}$ \\
Schnupfen & 105 & $92,4 \%$ & $\mathbf{p 0 , 0 1}$ \\
Halsschmerzen & 73 & $86,3 \%$ & n.s. \\
Fieber & 61 & $88,5 \%$ & n.s. \\
Kopfschmerzen & 57 & $94,7 \%$ & $\mathbf{p}<\mathbf{0 , 0 5}$ \\
Abgeschlagenheit & 42 & $88,1 \%$ & n.s. \\
Heiserkeit & 31 & $80,6 \%$ & n.s. \\
Gliederschmerzen & 30 & $96,7 \%$ & n.s. \\
Ohrenschmerzen & 24 & $83,3 \%$ & n.s. \\
Schmerzen NNH & 14 & $100,0 \%$ & n.s. \\
\hline
\end{tabular}

Das Auftreten von produktivem feuchtem Husten führte im Vergleich zum Auftreten eines unproduktiven trockenen Hustens zu einer signifikant erhöhten Verordnung von Husten- und Erkältungspräparaten $(p<0,05)$. Wenn ein Patient Husten mit gelblich-grünem Auswurf schilderte, wurde ebenfalls signifikant häufiger ein solches Präparat verschrieben als bei jemandem mit weißlichem Auswurf $(\mathrm{p}<0,05)$.

Pathologische Atemgeräusche $(93,3 \%$ der Patienten mit Verordnung von Husten- und Erkältungspräparaten) oder Nebengeräusche $(91,3 \%)$ waren ohne signifikanten Einfluss auf die Verordnung von Husten- und Erkältungspräparaten. Demgegenüber waren gerötete Tonsillen (90,4\% der Patienten mit Verordnung von Husten- und Erkältungspräparaten) signifikant mit der Gabe dieser Präparate verknüpft $(\mathrm{p}<0,05)$. Dabei ist zu berücksichtigen, dass das Symptom gerötete Tonsillen zwar mit einer hohen Rate an diesen Verordnungen assoziiert war, allerdings nur bei 18 von 125 Patienten (14,4\%) mit diesem Symptom auch die Diagnose einer akuten Tonsillitis gestellt wurde. Das Symptom gerötete Tonsillen wurde demnach von den Ärzten eher als eines im Rahmen eines oberen Atemwegsinfektes gewertet. Dies erklärt die Diskrepanz zur signifikant selteneren Verordnung beim Symptom Tonsillen mit Stippchen/ Belägen als Indikator einer akuten Tonsillitis $(\mathrm{p}<0,0001)$. Bei diesen Symptomen bestand eine signifikant geringere Verordnung von Husten- und Erkältungspräparaten (56,3\%). 
Beim Vergleich zwischen Patienten mit einem Lebensalter von 60 oder mehr Jahren bestand, bezogen auf die genannten Arzneien, statistisch kein Unterschied. Ebenso fanden sich keine Unterschiede zwischen Rentnern und Erwerbstätigen sowie zwischen Letzteren und NichtErwerbstätigen.

Zur weiteren Analyse relevanter Befunde wurde ergänzend eine multiple logistische Regressionsanalyse vorgenommen (Tabelle 20). Symptomatische Präparate wurden gehäuft bei den Symptomen Husten, Kopfschmerzen und Schnupfen sowie einem pathologischen Rachenbefund verordnet. Tabelle 20 zeigt die entsprechenden Ergebnisse der logistischen Regressionsanalyse. Folgende Variablen waren ohne Einfluss: pathologischer Otoskopiebefund, pathologischer Palpationsbefund der Nasennebenhöhlen, Auskultation pathologischer Atem- oder Nebengeräusche, pathologischer Palpationsbefund der Halslymphknoten, schmerzende Nasennebenhöhlen, Abgeschlagenheit, gerötete Tonsillen, mit Stippchen belegte Tonsillen, Fieber, Auswurf (sowohl gelblich-grünes Sputum als auch weißliches Sputum), Heiserkeit, Halsschmerzen, Ohrenschmerzen, Alter und Raucherstatus.

Tabelle 20: Abhängigkeit der Verordnung von Husten- und Erkältungspräparaten von Symptomen und Untersuchungsbefunden mittels multipler logistischer Regressionsanalyse

\begin{tabular}{lcc}
\hline \multicolumn{1}{c}{ Symptom/Befund } & OR & 95\%-CI \\
\hline Husten & 5,09 & $2,43-10,69$ \\
Kopfschmerzen & 2,43 & $1,02-5,83$ \\
Schnupfen & 2,39 & $1,2-4,76$ \\
pathologischer Rachenbefund & 2,16 & $1,11-4,2$ \\
\hline
\end{tabular}




\subsubsection{Praxischarakteristika als Einflussfaktoren auf die Verordnung von Husten- und Erkältungspräparaten}

Des Weiteren wurden mögliche Einflussfaktoren von Seiten der behandelnden Ärzte auf die Verordnung von Husten- und Erkältungspräparaten untersucht. Als einziger signifikanter Einflussparameter auf deren Verordnung konnte die Praxisgröße, gemessen an der abgerechneten Scheinzahl pro Quartal $(<1500$ und $>1500$ Scheine pro Quartal), errechnet werden. Ärzte aus Praxen mit mehr als 1500 Scheinen verordneten signifikant mehr Husten- und Erkältungspräparate als Ärzte aus Praxen mit einer Scheinzahl unter 1500 Scheinen. Dies konnte nur für die Gesamtheit der Diagnosen bewiesen werden. Das unterschiedliche Verordnungsverhalten lässt sich nicht mit divergierenden Altersstrukturen der Patienten erklären Patienten in Praxen mit weniger als 1500 Scheinen waren durchschnittlich 40,5 $( \pm 18,3)$ Jahre alt gegenüber einem Lebensalter von 39,1 ( $\pm 20,9)$ Jahren bei Praxen größer als 1500 Scheinen (FTest $0,179, \mathrm{p}<0,001$, t-Test $0,362, \mathrm{p}<0,001)$.

Ohne signifikanten Einfluss auf die Verordnung von Husten- und Erkältungspräparaten waren der Ort der Niederlassung (Brilon oder Hildesheim), das Alter der Praxisinhaber (<50 Jahre und $\geq 50$ Jahre), die Dauer der bisherigen Niederlassung ( $<15$ und $\geq 15$ Jahre Niederlassungsdauer) und die Niederlassung in Einzel- oder Gemeinschaftspraxen.

\subsubsection{Verordnung von Antitussiva und mögliche Einflussfaktoren auf die Verordnung}

Antitussiva werden zur Gruppe der Husten- und Erkältungspräparate gerechnet. Auf Grund ihrer gut abzugrenzenden Indikation sollen möglichen Einflussfaktoren auf die Verordnung bei Erstkontakten dargestellt werden. Insgesamt erhielten 59 Patienten (21,6\%) ein Antitussivum, dabei handelte es sich um 30 Erstkontakte (16,1\%). Wenn Patienten über unproduktiv trockenen Husten klagten, ergab sich keine signifikant höhere Antitussivaverordnung als bei solchen, die unter produktivem Husten litten.

Eine Anamnese bezüglich einer Zunahme des Hustens in der Nacht wurde nur bei 22 der 146 Betroffenen durchgeführt (15,1\%). Wenn sie darüber befragt wurden, gaben sie ohne Ausnahme 
an, dass sie unter verstärktem Husten in der Nacht litten. 14 Patienten $(63,6 \%)$ erhielten dann die Verordnung eines Antitussivums.

\subsection{Diagnostik und medikamentöse Therapie der 4 häufigsten Krankheitsbilder}

Wie bereits in Kapitel 3.5 beschrieben sind die am häufigsten gestellten Diagnosen sowohl bei Erst- als auch bei Folgekontakten der obere Atemwegsinfekt, die akute Bronchitis, die akute Tonsillitis und die akute Sinusitis. Die genannten Diagnosen hatten bei Erstkontakten einen Anteil von 81,4\% und bei Folgekontakten von 70,5\% an den insgesamt gestellten Diagnosen.

Wie in Kapitel 1.1 dargestellt wird die Hypothese einer einheitlichen Entität des oberen Atemwegsinfektes mit lediglich unterschiedlichen anatomischen Manifestationen diskutiert (Hueston et al. 2000 a, Glasziou und Del Mar 2002). Daher erfolgt in den anschließenden Kapiteln eine ausführliche Darstellung der Diagnostik und Therapie dieser 4 Krankheitsbilder um Unterschiede, aber auch Parallelen nachweisen zu können.

Ergänzend wurden mit dem statistischen Verfahren der multiplen logistischen Regressionsanalyse charakteristische Symptome und Untersuchungsbefunde in Bezug auf die 4 häufigsten Diagnosen analysiert. Dieses Verfahren wurde gewählt um herauszufinden, anhand welcher Symptome und Untersuchungsbefunde Ärzte ihre Diagnose stellten.

\subsubsection{Diagnostik und medikamentöse Therapie des oberen Atemwegsinfektes}

\subsubsection{Patientenanamnese}

Die Diagnose des oberen Atemwegsinfektes wurde bei 90 Patienten (48,4\%) mit Erstkontakt und $27(31,0 \%)$ mit Folgekontakt gestellt. Das Durchschnittsalter der Patienten betrug bei Erstkontakten 35,6 $( \pm 17,5)$ Jahre und bei Folgekontakten 39,70 $( \pm 19,9)$ Jahre. Bis zur Erstkonsultation des Arztes vergingen durchschnittlich 4,3 $( \pm 4,6)$ Tage und 9,89 $( \pm 8,4)$ Tage bis zur Folgekonsultation, und zwar gerechnet vom angegebenen Krankheitsbeginn. 
Patienten schilderten beim Erstkontakt 4,4 $( \pm 1,8)$ Symptome, beim Folgekontakt 3,3 $( \pm 1,4)$ Symptome. Das Symptom Husten wurde sowohl bei Erst- als auch bei Folgekontakten am häufigsten genannt, bei 38,9\% der Erstkontakte und 55,6\% der Folgekontakte war es mit Auswurf verbunden. Lagen Angaben zu dessen Farbe vor, so klagten 7,8\% der Erstkontakte und 18,5\% der Folgekontakte über gelblich-grünen Auswurf. Weißlicher Auswurf war bei 5,6\% der Erstkontakte und 18,5\% der Folgekontakte vorhanden. Des Weiteren litten Patienten häufig unter Schnupfen (70,0\% der Erstkontakte und 44,4\% der Folgekontakte), Kopfschmerzen (37,4\%, bzw. $14,8 \%$ ) und Halsschmerzen (37,4\%, bzw. 29,6\%).

\subsubsection{Körperliche Untersuchung}

Tabelle 21 zeigt die durchgeführten körperlichen Untersuchungen. Bei mehr als 90\% der Erstkontakte und knapp 60\% der Folgekontakte wurde eine Inspektion des Mund- und Rachenraumes durchgeführt. Die Auskultation der Lunge war mit fast $80 \%$ bei Erst- und Folgekontakten ebenfalls eine häufig angewandte Diagnostik.

Tabelle 21: Körperliche Untersuchung beim oberen Atemwegsinfekt (Anzahl der Patienten absolut und in \%)

\begin{tabular}{|c|c|c|c|c|}
\hline \multirow{2}{*}{ Art der körperlichen Untersuchung } & \multicolumn{2}{|c|}{ Erstkontakte } & \multicolumn{2}{|c|}{ Folgekontakte } \\
\hline & $\mathbf{n}$ & (\%) & $\mathbf{n}$ & $(\%)$ \\
\hline Inspektion des Mund- und Rachenraumes & 82 & $(91,1 \%)$ & 16 & $(59,3 \%)$ \\
\hline Auskultation der Lunge & 78 & $(86,7 \%)$ & 21 & $(77,8 \%)$ \\
\hline Otoskopie & 35 & $(38,9 \%)$ & 6 & $(22,2 \%)$ \\
\hline Palpation der Halslymphknoten & 33 & $(36,7 \%)$ & 3 & $(11,1 \%)$ \\
\hline Palpation der NNH & 30 & $(33,3 \%)$ & 5 & $(18,5 \%)$ \\
\hline Perkussion der Lunge & 18 & $(20,0 \%)$ & 3 & $(11,1 \%)$ \\
\hline Stimmfremitus & 8 & $(8,9 \%)$ & 0 & \\
\hline
\end{tabular}




\subsubsection{Abhängigkeit der Diagnosestellung von Symptomen und Untersuchungsbefunden}

Mit dem statistischen Verfahren der multiplen logistischen Regressionsanalyse konnte errechnet werden, dass die Diagnose des oberen Atemwegsinfektes gehäuft gestellt wurde, wenn die Symptome Schnupfen, Husten, Ohrenschmerzen und Heiserkeit vorlagen (Tabelle 22).

Pathologische körperliche Untersuchungsbefunde wie die Auskultation pathologischer Nebengeräusche und Atemgeräusche sowie der pathologische Palpationsbefund der Nasennebenhöhlen schlossen die Diagnose eher aus. Folgende Variablen waren ohne Einfluss: Abgeschlagenheit, Halsschmerzen, Fieber, Kopfschmerzen, pathologischer Befund bei der Inspektion des Mund-Rachenraumes (gerötete Rachenhinterwand, mit Stippchen belegte Tonsillen) pathologischer Otoskopiebefund, pathologischer Palpationsbefund der Halslymphknoten, Alter und Raucherstatus.

Tabelle 22: Abhängigkeit der Diagnosestellung oberer Atemwegsinfekt von Symptomen und Untersuchungsbefunden

\begin{tabular}{lcc}
\hline \multicolumn{1}{c}{ Symptom/Untersuchungsbefund } & OR & $\mathbf{9 5 \% - C I}$ \\
\hline Schnupfen & 3,35 & $1,95-5,77$ \\
Husten & 2,94 & $1,33-6,50$ \\
Ohrenschmerzen & 2,92 & $1,29-6,59$ \\
Heiserkeit & 2,63 & $1,27-5,45$ \\
Auskultation pathologischer Nebengeräusche & 0,03 & $0,01-0,10$ \\
pathologischer Palpationsbefund der Nasennebenhöhlen & 0,11 & $0,04-0,28$ \\
Auskultation pathologischer Atemgeräusche & 0,14 & $0,05-0,47$ \\
Auswurf & 0,33 & $0,18-0,61$ \\
Schmerzen Nasennebenhöhlen & 0,34 & $0,12-0,94$ \\
\hline
\end{tabular}

\subsubsection{Medikamentöse Therapie}

Den größten Anteil der Verordnungen nahmen Verordnungen aus der therapeutischen Hauptgruppe der Husten- und Erkältungspräparate ein. Wie in Abbildung 2 ersichtlich, erhielten 90\% der Erstkontakte Medikamente aus dieser Gruppe; hiervon waren 71,1\% Expektoranzien. 


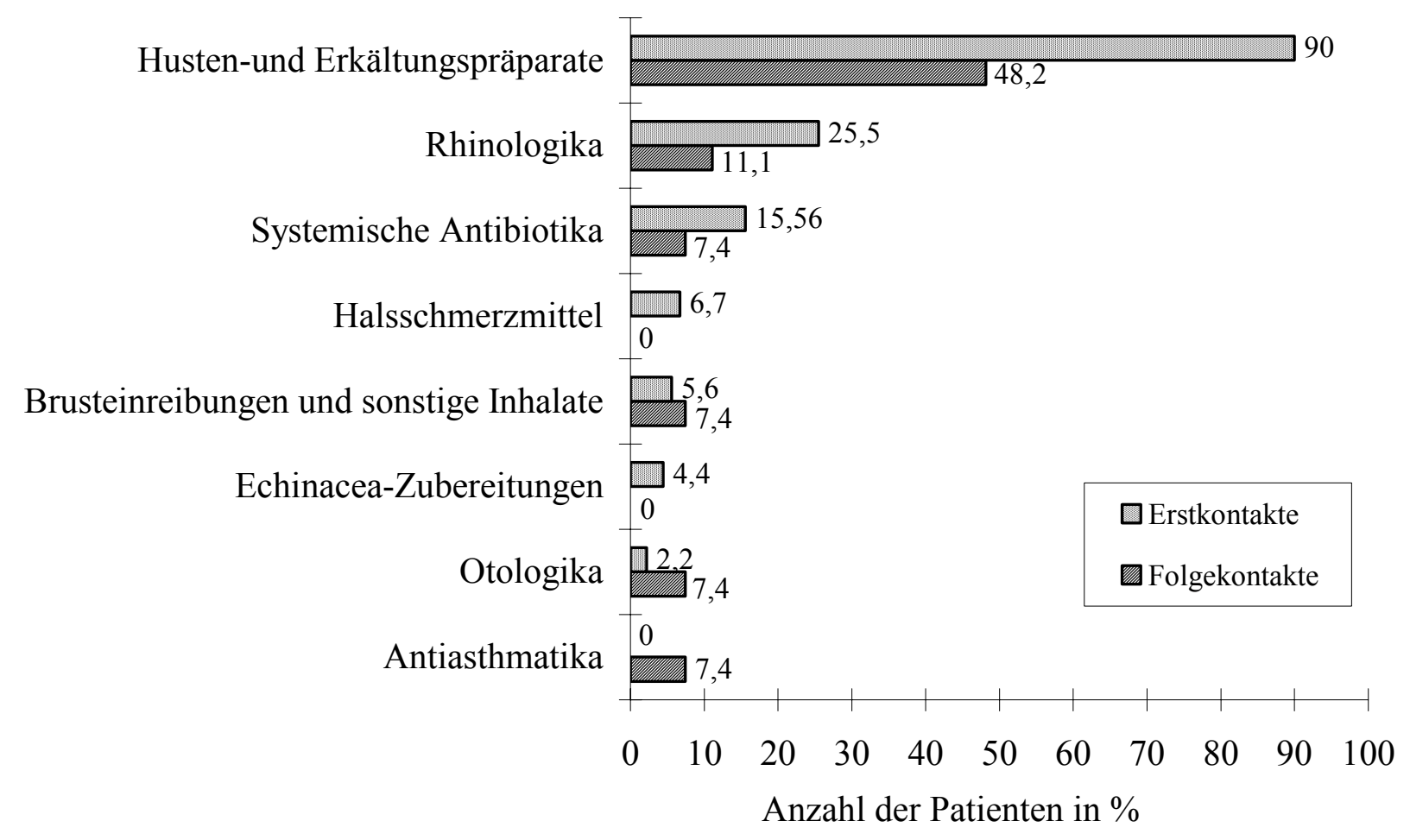

Abbildung 2: Medikamentöse Therapie des oberen Atemwegsinfektes

Tabelle 23 zeigt die Verteilung der Antibiotikaverordnungen nach ihren pharmakologischen Hauptgruppen und ihrer chemischen Substanz gemäß ATC-Code. Am häufigsten wurde die pharmakologische Hauptgruppe der Makrolide verordnet. Auf Roxithromycin wurde mit 5 Verordnungen beim Erstkontakt als chemische Substanz am meisten zurückgegriffen.

Tabelle 23: Antibiotikaverordnungen beim oberen Atemwegsinfekt (Patientenanzahl absolut)

\begin{tabular}{llcc}
\hline \multicolumn{1}{c}{$\begin{array}{c}\text { Pharmakologische } \\
\text { Hauptgruppe }\end{array}$} & \multicolumn{1}{c}{ Chemische Substanz } & Erstkontakte & Folgekontakte \\
\hline $\begin{array}{l}\text { Expektoranzien-Kombination } \\
\text { mit Antibiotika }\end{array}$ & $\begin{array}{l}\text { Doxycyclin- } \\
\text { Kombinationen }\end{array}$ & 3 & 0 \\
Breitbandpenicilline & Amoxicillin & 2 & 1 \\
Cephalosporine & Cefaclor & 2 & 0 \\
& Cefuroximaxetil & 1 & 0 \\
Makrolide & Erythromycin & 0 & 1 \\
& Roxithromycin & 5 & 0 \\
& Azithromycin & 1 & 0 \\
\hline
\end{tabular}




\subsubsection{Diagnostik und medikamentöse Therapie der akuten Bronchitis}

\subsubsection{Patientenanamnese}

Die Diagnose der akuten Bronchitis wurde bei 46 Patienten mit Erstkonsultation (24,7\%) und 24 Patienten mit Folgekonsultation (27,6\%) gestellt. Ihr Durchschnittsalter betrug bei Erstkontakten $44,7( \pm 21,6)$ Jahre und bei Folgekontakten 54,1 $( \pm 19,8)$ Jahre. Bis zur Konsultation des Arztes vergingen durchschnittlich 6,4 $( \pm 5,1)$ Tage bei Erstkontakten und 10,3 $( \pm 6,9)$ Tage bei Folgekontakten, gerechnet vom angegebenen Krankheitsbeginn. Patienten schilderten beim Erstkontakt 4,4 $( \pm 1,7)$ Symptome, beim Folgekontakt 2,3 $( \pm 1,1)$ Symptome.

Husten war bei allen Erstkontakten und bei 95,8\% der Folgekontakte das auffallendste Symptom, wobei 78,3\% der Erstkontakte und 83,3\% der Folgekontakte auch über Auswurf klagten. Wenn Angaben zu dessen Farbe gemacht wurden, handelte es sich bei 54,4\% derjenigen mit Erstkontakt um gelblich-grünen und bei 10,9\% um weißlichen Auswurf. Folgekontakte klagten häufiger über weißlichen $(33,3 \%)$ als über gelblich-grünen Auswurf (25,0\%). Erstkontakte fühlten sich oft durch Schnupfen (54,4\%), Fieber (37,0\%), Abgeschlagenheit $(34,8 \%)$ und Halsschmerzen (30,4\%) gesundheitlich beeinträchtigt. Bei Folgekontakten wurden Abgeschlagenheit (20,8\%), Schnupfen (16,7\%), Kreislaufbeschwerden (16,7\%) und nächtlicher Husten $(16,7 \%)$ als weitere Symptome genannt.

\subsubsection{Körperliche Untersuchung}

Tabelle 24 zeigt die Darstellung der durchgeführten körperlichen Untersuchungen. Als häufigste Untersuchung erfolgte bei 97,8\% der Erstkontakte und 95,8\% der Folgekontakte eine Auskultation der Lunge. 
Tabelle 24: Körperliche Untersuchung bei akuter Bronchitis (Anzahl der Patienten absolut und in $\%)$

\begin{tabular}{lrcrc}
\hline \multicolumn{1}{c}{ Art der körperlichen Untersuchung } & \multicolumn{2}{c}{$\begin{array}{c}\text { Erstkontakte } \\
\text { n }\end{array}$} & \multicolumn{2}{c}{ Folgekontakte } \\
& $\mathbf{( \% )}$ & n & (\%) \\
\hline Auskultation der Lunge & 45 & $(97,8 \%)$ & 23 & $(95,8 \%)$ \\
Inspektion des Mund- und Rachenraumes & 36 & $(78,3 \%)$ & 8 & $(33,3 \%)$ \\
Palpation der Halslymphknoten & 11 & $(23,9 \%)$ & 3 & $(12,5 \%)$ \\
Otoskopie & 11 & $(23,9 \%)$ & 2 & $(8,3 \%)$ \\
Palpation der NNH & 9 & $(19,6 \%)$ & 4 & $(16,7 \%)$ \\
Perkussion der Lunge & 4 & $(8,7 \%)$ & 2 & $(8,3 \%)$ \\
\hline
\end{tabular}

\subsubsection{Abhängigkeit der Diagnosestellung von Symptomen und Untersuchungsbefunden}

Nur ein Patient mit der Diagnose einer akuten Bronchitis klagte nicht über Husten. Daher ließ sich ein stabiles Regressionsmodell erst nach Auslassung dieses Symptoms errechnen. Wie aus Tabelle 25 ersichtlich war die Diagnose der akuten Bronchitis, neben dem nahezu immer vorliegendem Symptom Husten, vor allem mit pathologischen Lungenuntersuchungsbefunden assoziiert. Weiterhin bestand eine Verknüpfung zu den Symptomen Abgeschlagenheit und Vorhandensein von Auswurf. Bei Rauchern wurde die Diagnose signifikant seltener gestellt. Folgende Variablen waren ohne Einfluss: Auswurf (sowohl gelblich-grünes als auch weißliches Sputum), Fieber, Schnupfen, Ohrenschmerzen, Heiserkeit, Kopfschmerzen, Halsschmerzen, pathologischer Palpationsbefund der Halslymphknoten, schmerzende Nasennebenhöhlen, pathologischer Otoskopiebefund, pathologischer Befund bei der Inspektion des MundRachenraumes (gerötete Rachenhinterwand, gerötete Tonsillen, mit Stippchen belegte Tonsillen) und Alter.

Tabelle 25: Abhängigkeit der Diagnosestellung akute Bronchitis von Symptomen und Untersuchungsbefunden

\begin{tabular}{lcc}
\hline \multicolumn{1}{c}{ Symptom/Befund } & OR & 95\%-CI \\
\hline Auskultation pathologischer Nebengeräusche & 12,01 & $5,25-27,50$ \\
Auskultation pathologischer Atemgeräusche & 5,69 & $2,12-15,27$ \\
Abgeschlagenheit & 3,53 & $1,53-8,17$ \\
Vorhandensein von Auswurf & 2,74 & $1,22-6,18$ \\
Raucher & 0,28 & $0,10-0,76$ \\
\hline
\end{tabular}




\subsubsection{Medikamentöse Therapie}

93,5\% der Erstkontakte wurde ein Medikament aus der Gruppe der Husten- und Erkältungspräparate verordnet, bei den Folgekontakten waren es 62,5\%. Die pharmakologische Hauptgruppe der Expektoranzien machte hierbei den größten Teil aus $(84,8 \%$ der Erstkontakte und $62,5 \%$ der Folgekontakte).

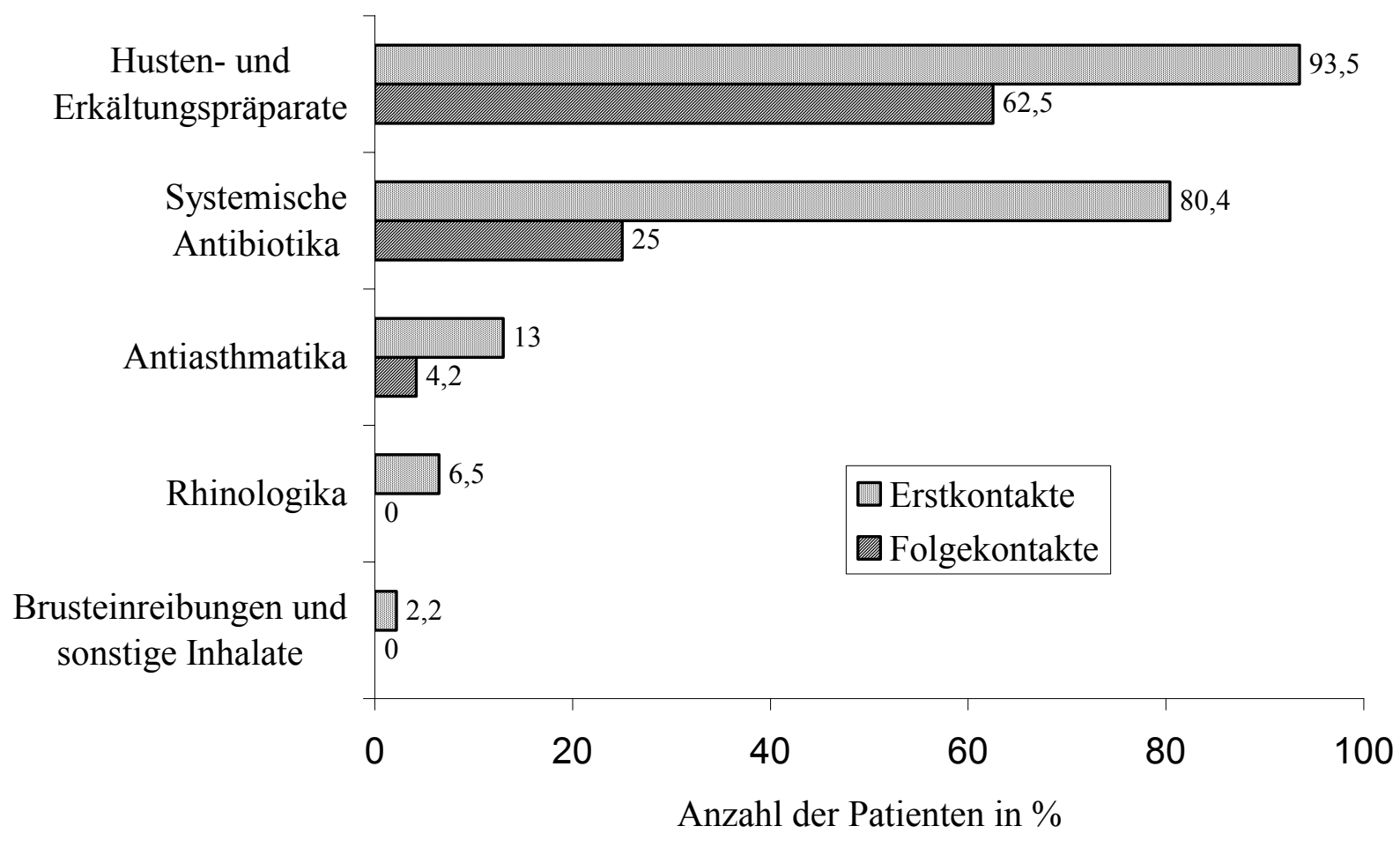

Abbildung 3: Medikamentöse Therapie der akuten Bronchitis

Tabelle 26 zeigt die Verteilung der Antibiotikaverordnungen nach ihren pharmakologischen Hauptgruppen und ihrer chemischen Substanz gemäß ATC-Code. Makrolide wurde am häufigsten verordnet (28,6\% der Patienten). In dieser Gruppe befinden sich mit Roxithromycin (8 Verordnungen) und Azithromycin (7 Verordnungen) die am häufigsten verordneten chemischen Substanzen. Insbesondere bei der Diagnose der akuten Bronchitis fällt der verstärkte Einsatz von Expektoranzien-Kombinationen mit Antibiotika auf (5 Verordnungen beim Erstkontakt, 2 Verordnungen beim Folgekontakt). 
Tabelle 26: Antibiotikaverordnungen bei akuter Bronchitis (Patientenanzahl absolut)

\begin{tabular}{llcc}
\hline \multicolumn{1}{c}{$\begin{array}{c}\text { Pharmakologische } \\
\text { Hauptgruppe }\end{array}$} & \multicolumn{1}{c}{ Chemische Substanz } & Erstkontakte & Folgekontakte \\
\hline $\begin{array}{l}\text { Expektoranzien-Kombination } \\
\text { mit Antibiotika }\end{array}$ & $\begin{array}{l}\text { Doxycyclin- } \\
\text { Kombinationen }\end{array}$ & 5 & 2 \\
Tetracycline & Doxycyclin & 1 & 1 \\
Breitbandpenicilline & Amoxicillin & 6 & 1 \\
Cephalosporine & Cefaclor & 1 & 0 \\
& Cefixim & 1 & 0 \\
& Cefpodoxim & 1 & 0 \\
Makrolide & Cefuroximaxetil & 2 & 0 \\
& Erythromycin & 1 & 1 \\
& Roxithromycin & 8 & 1 \\
Lincosamide & Clarithromycin & 2 & 0 \\
Chinolonantibiotika & Azithromycin & 7 & 0 \\
\hline
\end{tabular}

\subsubsection{Diagnostik und medikamentöse Therapie der akuten Tonsillitis}

\subsubsection{Patientenanamnese}

Bei 21 Patienten (11,3\%) mit Erstkontakt und 9 Patienten (10,3\%) mit Folgekontakt wurde eine akute Tonsillitis diagnostiziert. Das Durchschnittsalter betrug bei Erstkontakten $34,6( \pm 15,9)$ Jahre und bei Folgekontakten $41,9( \pm 14,9)$ Jahre. Bis zur Konsultation des Arztes vergingen durchschnittlich 4,5 $( \pm 4,0)$ Tage bei Erstkontakten und 12,44 $( \pm 9,0)$ Tage bei Folgekontakten, gerechnet vom angegebenen Krankheitsbeginn.

Patienten schilderten beim Erstkontakt 3,0 $( \pm 1,4)$ und beim Folgekontakt 3,4 $( \pm 2,5)$ Symptome. Erwartungsgemäß wurden bei der Diagnose der akuten Tonsillitis am häufigsten Halsschmerzen angegeben. Des Weiteren klagten 42,9\% der Patienten mit Erstkontakt und 22,2\% mit Folgekontakt über Husten. Erstkontakte schilderten Fieber (38,1\%) und schmerzende Halslymphknoten $(23,8 \%)$ als weitere häufige Beschwerden. Ein Drittel der Folgekontakte klagte 
ebenfalls über schmerzende Halslymphknoten und die Symptome Schnupfen, Heiserkeit, Ohrenschmerzen und Abgeschlagenheit (jeweils 22,2\%).

\subsubsection{Körperliche Untersuchung}

Tabelle 27 listet die durchgeführten körperlichen Untersuchungen auf. Bei allen Erst- und Folgekontakten wurde der Mund- und Rachenraumes inspiziert. Eine weitere, vielfach vorgenommene Untersuchung stellte die Auskultation der Lunge dar, die bei 42,9\% der Erstkontakte und 55,6\% der Folgekontakte für notwendig erachtet wurde.

Tabelle 27: Körperliche Untersuchung bei akuter Tonsillitis (Anzahl der Patienten absolut und in $\%)$

\begin{tabular}{lrrrr}
\hline Art der körperlichen Untersuchung & \multicolumn{2}{c}{$\begin{array}{c}\text { Erstkontakte } \\
\text { n }\end{array}$} & $\begin{array}{r}\text { Folgekontakte } \\
\text { (\%) }\end{array}$ & n \\
& $\mathbf{( \% )}$ \\
\hline Inspektion des Mund- und Rachenraumes & 21 & $(100,0 \%)$ & 9 & $(100,0 \%)$ \\
Auskultation der Lunge & 9 & $(42,9 \%)$ & 5 & $(55,6 \%)$ \\
Palpation der Halslymphknoten & 7 & $(33,3 \%)$ & 3 & $(33,3 \%)$ \\
Otoskopie & 6 & $(28,6 \%)$ & 3 & $(33,3 \%)$ \\
Palpation der NNH & 3 & $(14,3 \%)$ & 2 & $(22,2 \%)$ \\
Perkussion der Lunge & 3 & $(14,3 \%)$ & 0 & \\
\hline
\end{tabular}

\subsubsection{Abhängigkeit der Diagnosestellung von Symptomen und Untersuchungsbefunden}

Die durchgeführte multiple logistische Regressionsanalyse zeigt, dass die Diagnose einer akuten Tonsillitis mit dem Symptom Halsschmerzen und pathologischen Rachenbefunden bei der Inspektion des Nasen-Rachen-Raumes sowie einem pathologischen Befund bei der Palpation der Halslymphknoten assoziiert war (Tabelle 28). Die Symptome Schnupfen und Husten waren dagegen negativ mit der Diagnosestellung verknüpft. Ohne Einfluss waren die Variablen: Ohrenschmerzen, Auswurf (sowohl gelblich-grünes als auch weißliches Sputum), Kopfschmerzen, Abgeschlagenheit, Fieber, Heiserkeit, schmerzende Nasennebenhöhlen, pathologischer Otoskopiebefund, Auskultation pathologischer Atem- oder Nebengeräusche, pathologischer Palpationsbefund der Nasennebenhöhlen und Raucherstatus. 
Tabelle 28: Abhängigkeit der Diagnosestellung akute Tonsillitis von Symptomen und Untersuchungsbefunden

\begin{tabular}{|c|c|c|}
\hline Symptom/Befund & OR & $95 \%-$-CI \\
\hline Halsschmerzen & 44,53 & $5,63-352,03$ \\
\hline pathologischer Rachenbefund & 13,32 & $1,74-102,07$ \\
\hline $\begin{array}{l}\text { pathologischer Palpationsbefund der } \\
\text { Halslymphknoten }\end{array}$ & 4,9 & $1,8-13,36$ \\
\hline Schnupfen & 0,06 & $0,02-0,26$ \\
\hline Husten & 0,13 & $0,04-0,39$ \\
\hline
\end{tabular}

\subsubsection{Medikamentöse Therapie}

Am häufigsten wurden bei der Diagnose der akuten Tonsillitis Antibiotika verordnet (85,7\% der Erstkontakte und 55,6\% der Folgekontakte). An 2. Stelle folgten Verschreibungen aus der Gruppe der Husten- und Erkältungspräparate (57,1\% der Erstkontakte und 33,3\% der Folgekontakte). Die akute Tonsillitis ist das einzige Krankheitsbild, bei dem die Verordnung von Antibiotika und nicht wie bei den anderen 3 beschriebenen Krankheitsbildern, die Gruppe der Husten- und Erkältungspräparate an 1. Stelle steht.

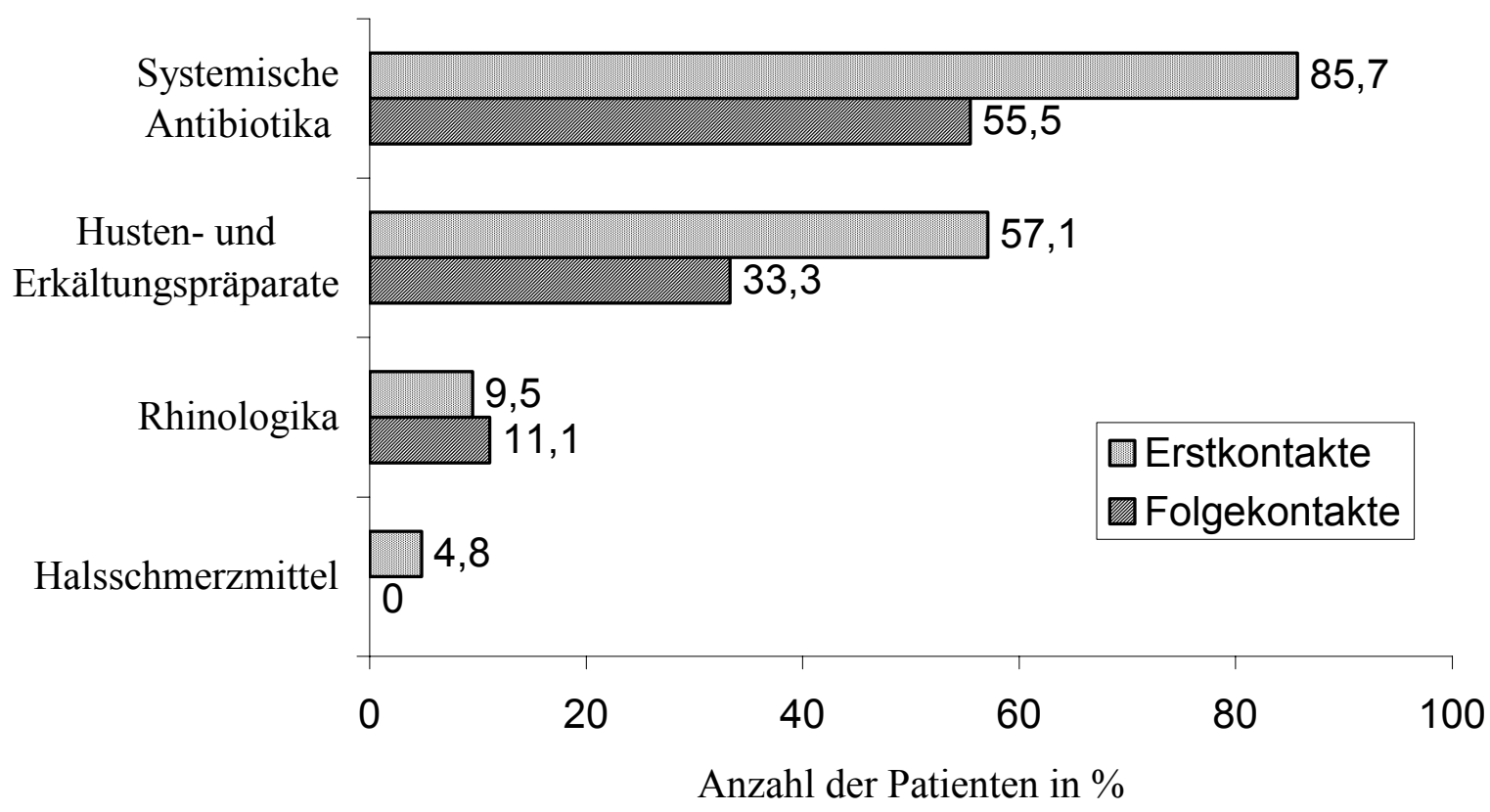

Abbildung 4: Medikamentöse Therapie der akuten Tonsillitis 
Tabelle 29 demonstriert die Verteilung der Antibiotikaverordnungen nach ihren pharmakologischen Hauptgruppen und ihrer chemischen Substanz gemäß ATC-Code. 47,6\% der Erstkontakte und 22,2\% der Folgekontakte mit der Diagnose einer akuten Tonsillitis bekamen ein Penicillinpräparat. Des Weiteren wurden Antibiotika aus der Gruppe der Makrolide (23,3\%) und Cephalosporine $(6,7 \%)$ eingesetzt. Die am häufigsten verschriebene chemische Substanz war Phenoxymethylpenicillin.

Tabelle 29: Antibiotikaverordnungen bei akuter Tonsillitis (Patientenanzahl absolut)

\begin{tabular}{llcc}
\hline $\begin{array}{c}\text { Pharmakologische } \\
\text { Hauptgruppe }\end{array}$ & Chemische Substanz & $\begin{array}{c}\text { bei Erstkontakt } \\
\text { verordnet }\end{array}$ & $\begin{array}{c}\text { bei Folgekontakt } \\
\text { verordnet }\end{array}$ \\
\hline Breitbandpenicilline & Ampicillin & 1 & 0 \\
Betalactamase- & Amoxicillin & 1 & 0 \\
sensitive Penicilline & Phenoxymethyl- & 6 & 2 \\
& Penicillin & 1 & 0 \\
& Propicillin & 1 & 0 \\
Cenzylpenicillin- & Kombinationen & 1 & 0 \\
Makrolide & Cefpodoxim & 0 & 1 \\
& Cefuroximaxetil & 4 & 1 \\
& Roxithromycin & 0 & 1 \\
\hline
\end{tabular}

\subsubsection{Diagnostik und medikamentöse Therapie der akuten Sinusitis}

\subsubsection{Patientenanamnese}

Unter einer akuten Sinusitis litten 19 Patienten (10,2\%) bei der Erstkonsultation und 14 Patienten $(16,1 \%)$ bei der Folgekonsultation. Das Durchschnittsalter der Patienten betrug bei Erstkontakten 46,1 $( \pm 15,8)$ Jahre und bei Folgekontakten 43,5 $( \pm 16,0)$ Jahre. Bis zur Konsultation des Arztes vergingen durchschnittlich 15,5 $( \pm 22,5)$ Tage bei Erstkontakten und 8,6 $( \pm 6,0)$ Tage bei Folgekontakten, geht man vom angegebenen Krankheitsbeginn aus. Patienten schilderten beim Erstkontakt 3,8 $( \pm 1,3)$ und beim Folgekontakt $4,9( \pm 1,8)$ Symptome. Erstkontakte hoben besonders die Symptome Schnupfen (79,0\%), Kopfschmerzen (52,6\%), Husten (52,6\%), Husten 
mit Auswurf (31,6\%) und Schmerzen im Bereich der Nasennebenhöhlen hervor. Bei Folgekontakten waren die Symptome Schnupfen (71,4\%), Kopfschmerzen (64,3\%), Schmerzen im Bereich der Nasennebenhöhlen (57,1\%) und Halsschmerzen (35,7\%) führend. Zahnschmerzen wurden von 10,5\% der Erstkontakte und 21,4\% der Folgekontakte geschildert.

\subsubsection{Körperliche Untersuchung}

In Tabelle 30 erfolgt eine Darstellung der durchgeführten körperlichen Untersuchungen. Bei allen Patienten wurden die Nasennebenhöhlen palpiert. Eine weitere, sehr häufige Untersuchung stellte die Inspektion des Mund- und Rachenraumes dar.

Tabelle 30: Körperliche Untersuchung bei akuter Sinusitis (Anzahl der Patienten absolut und in $\%)$

\begin{tabular}{lrrrr}
\hline \multicolumn{1}{c}{ Art der körperlichen Untersuchung } & \multicolumn{2}{c}{$\begin{array}{c}\text { Erstkontakte } \\
\text { n }\end{array}$} & \multicolumn{2}{r}{ Folgekontakte } \\
& $\mathbf{n}$ & $\mathbf{\text { n }}$ & $\mathbf{( \% )}$ \\
\hline Palpation der NNH & 19 & $(100,0 \%)$ & 14 & $(100,0 \%)$ \\
Inspektion des Mund- und Rachenraumes & 17 & $(89,5 \%)$ & 13 & $(92,9 \%)$ \\
Otoskopie & 11 & $(57,9 \%)$ & 6 & $(42,9 \%)$ \\
Auskultation der Lunge & 11 & $(57,9 \%)$ & 10 & $(71,4 \%)$ \\
Palpation der Halslymphknoten & 3 & $(15,8 \%)$ & 4 & $(28,6 \%)$ \\
Perkussion der Lunge & 2 & $(10,5 \%)$ & 2 & $(14,3 \%)$ \\
\hline
\end{tabular}

\subsubsection{Abhängigkeit der Diagnosestellung von Symptomen und Untersuchungsbefunden}

Die Diagnosestellung der akuten Sinusitis war nahezu vollständig mit der Feststellung eines pathologischen Untersuchungsbefundes bei der Palpation der Nasennebenhöhlen verknüpft. So wiesen nur zwei Patienten keinen derartigen Befund auf. Ein stabiles logistisches Regressionsmodell ließ sich erst unter Auslassung dieser Variable erstellen. Danach konnte eine starke Assoziation zu schmerzenden Nasennebenhöhlen (OR 20,03, 95\%-CI: 6,39-62,82) und Kopfschmerzen (OR 4,12, 95\%-CI: 1,56-10,85) feststellt werden, während das Symptom Husten negativ mit der Diagnose einer akuten Sinusitis verknüpft war (OR 0,35, 95\%-CI: 0,12-0,96). Folgende Variablen waren ohne Einfluss: Auswurf (sowohl gelblich-grünes als auch weißliches 
Sputum), Abgeschlagenheit, Halsschmerzen, Fieber, Heiserkeit, Schnupfen, Auskultation pathologischer Atem- oder Nebengeräusche, pathologischer Otoskopiebefund, pathologischer Befund bei der Inspektion des Mund-Rachenraumes (gerötete Rachenhinterwand), pathologischer Palpationsbefund der Halslymphknoten, Alter und Raucherstatus.

\subsubsection{Medikamentöse Therapie}

Fast 95\% der Erstkontakte wurde ein Präparat aus der Gruppe der Husten- und Erkältungspräparate verschrieben, bei den Folgekontakten lag der Prozentsatz bei 57,1\%. Am zweithäufigsten wurden Antibiotika verordnet.

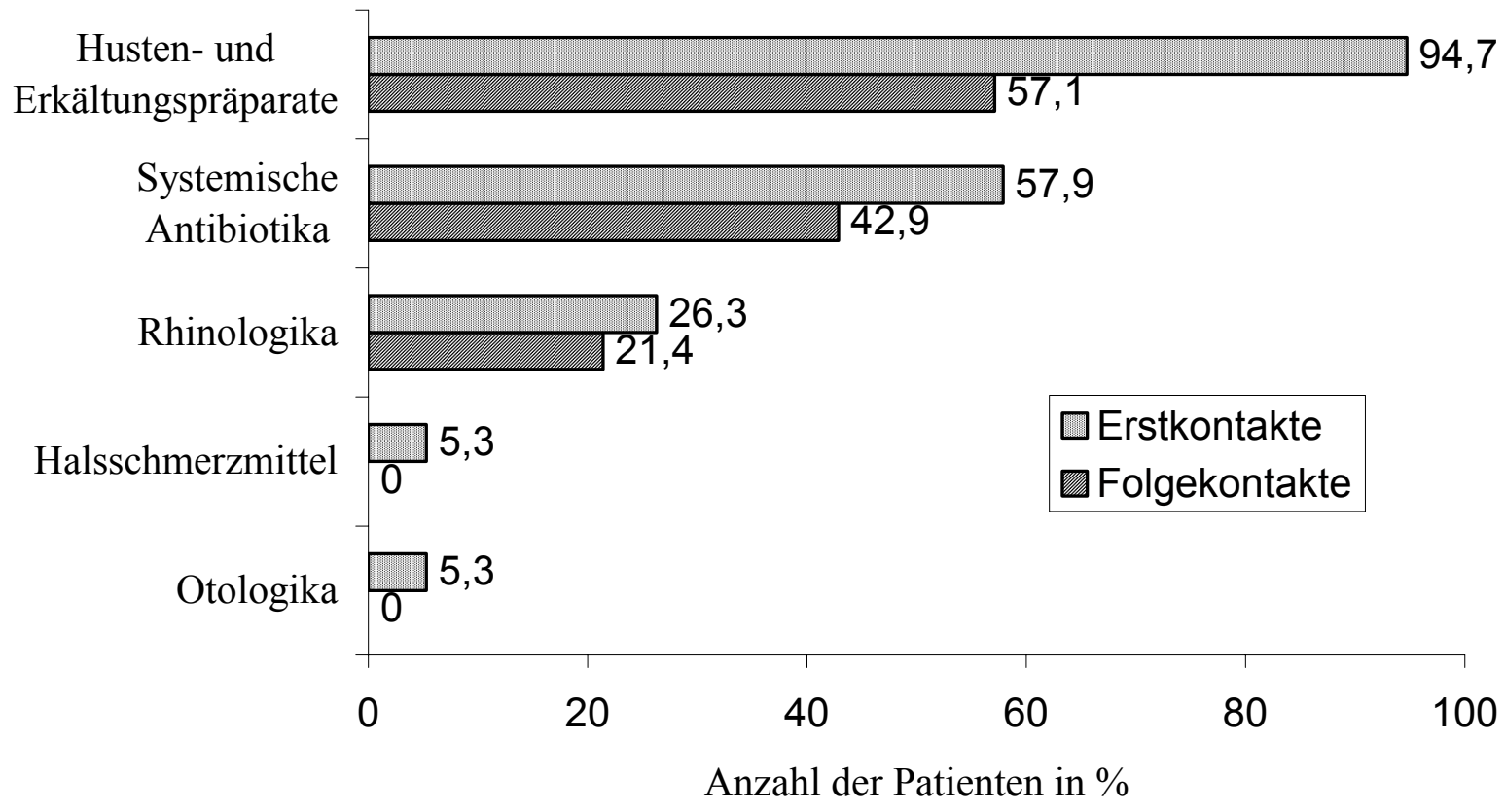

Abbildung 5: Medikamentöse Therapie der akuten Sinusitis

Wie beim oberen Atemwegsinfekt und der akuten Bronchitis bezogen sich auch bei der akuten Sinusitis die meisten Verschreibungen auf die Gruppe der Husten- und Erkältungspräparate. Eine weitere Unterteilung in die pharmakologischen Hauptgruppen ergab, dass 89,5\% der Erstkontakte und 50\% der Folgekontakte Expektoranzien erhielten. 
Tabelle 31 zeigt die Verteilung der Antibiotikaverordnungen nach ihren pharmakologischen Hauptgruppen und ihrer chemischen Substanz gemäß ATC-Code. Am beliebtesten war die Verordnung von Expektoranzien-Kombinationen mit Antibiotika.

Tabelle 31: Antibiotikaverordnungen bei akuter Sinusitis (Patientenanzahl absolut)

\begin{tabular}{|c|c|c|c|}
\hline $\begin{array}{l}\text { Pharmakologische } \\
\text { Hauptgruppe }\end{array}$ & Chemische Substanz & Erstkontakte & Folgekontakte \\
\hline $\begin{array}{l}\text { Expektoranzien- } \\
\text { Kombination mit Antibiotika }\end{array}$ & Doxycyclin-Kombinationen & 4 & 0 \\
\hline Tetracycline & Doxycyclin & 0 & 2 \\
\hline Breitbandpenicilline & Amoxicillin & 1 & 2 \\
\hline $\begin{array}{l}\text { Betalactamase-sensitive } \\
\text { Penicilline }\end{array}$ & Propicillin & 1 & 0 \\
\hline Cephalosporine & Loracarbef & 1 & 0 \\
\hline Makrolide & Erythromycin & 0 & 1 \\
\hline & Roxithromycin & 1 & 1 \\
\hline & Azithromycin & 2 & 0 \\
\hline Lincosamide & Clindamycin & 1 & 0 \\
\hline
\end{tabular}




\section{Diskussion}

\subsection{Methode}

Die vorliegende Untersuchung wurde nach der Methode der teilnehmenden Beobachtung durchgeführt. Ziel war es das alltägliche Vorgehen der Ärzte bei der Diagnostik und Therapie weitestgehend ohne Beeinflussung von außen zu dokumentieren. Lediglich die Angaben zur Person wurden von den Patienten erfragt.

Dieses Konzept hat seine Wurzeln in der sozialen und kulturellen Anthropologie der frühen 60erJahre. Die besondere Stärke der Methode ist die Miteinbeziehung der Aktivitäten und Interaktionen des Settings (in diesem Fall die Hausarztpraxis) auf das zu untersuchende Verhalten (hier das hausärztliche Vorgehen bei Atemwegsinfekten) (Bogdewic 1992). Dabei weist dieses Verfahren spezifische Vorteile auf (Bogdewic 1992):

- Nach Ablauf einer gewissen Zeitspanne werden die Beobachteten zunehmend weniger durch den Beobachter beeinflusst. Sie zeigen dann eher eine Gewöhnung als eine Anpassung an die besondere Situation. Die Beobachtung kommt dem üblichen Verhalten somit relativ nahe.

- Fragestellungen müssen nicht vorab, z.B. bei der Konstruktion eines Fragebogens entwickelt werden, sondern können aus dem Beobachtungsmaterial abgeleitet werden.

- Die zeitliche Abfolge des Verhaltens und äußere Einflussfaktoren, die auf das beobachtete Verhalten einwirken, können identifiziert werden, da die Beobachtung im alltäglichen Kontext erfolgt.

Obwohl Bogdewic (1992) davon ausgeht, dass es im zeitlichen Rahmen der Beobachtung eher zu einer Gewöhnung als zu einer Anpassung kommt, so sind sich sowohl Arzt als auch Patient zumindest anfangs der besonderen Situation bewusst, was nach anderen Autoren zweifellos eine mögliche Beeinflussung darstellt (Howie 1989). Auch sollte davon ausgegangen werden, dass sich speziell der Arzt einer Beobachtung und somit auch einer Beurteilung ausgesetzt sieht, was ihn zu einem von seinem üblichen Vorgehen abweichendem Verhalten führen könnte (Howie 
1989). Insofern kann nicht unreflektiert davon ausgegangen werden, dass die hier erhobenen Ergebnisse ein unverfälschtes Bild des alltäglichen Vorgehens liefern.

Bezogen auf die vorliegenden Daten können folgende Punkte als Vorteile des gewählten Studiendesigns gelten:

- Bei der Anamnese kamen tatsächlich nur die vom Arzt oder Patienten als vorrangig betrachteten Symptome zur Sprache.

- Die bei standardisierten Fragebögen bestehende Gefahr, dass Patienten erst durch die Befragung auf weitere Symptome aufmerksam gemacht werden, wurde vermieden.

- Wenn alternativ die Ärzte einen Dokumentationsbogen nach der Konsultation ausgefüllt hätten, wäre durch die Vorgaben des Bogens ebenfalls eine Beeinflussung möglich gewesen.

Als nachteilig können sich folgende Punkte auswirken:

- Ein gewisser "Vorführeffekt" konnte, insbesondere bei den körperlichen Untersuchungen und den Verordnungen, nicht ausschlossen werden.

- Möglicherweise wurden aus diesem Grund mehr Untersuchungen durchgeführt, mehr (oder auch weniger) Verordnungen ausgestellt und mehr Patienten wiedereinbestellt als sonst üblich, was mit den erhobenen Daten jedoch nicht zu beweisen ist.

Während der Konsultationen gab es keine Probleme mit der Erstellung der Dokumentation. Alle in Frage kommenden Patienten erklärten sich bereit an der Studie teilzunehmen. Die Anzahl der dokumentierten Patienten variierte zwischen 4 und maximal 17 pro Tag (Prävalenz 21,4\% $( \pm 6,4 \%)$ der Gesamtkonsultationen). Sicherlich ist es jedoch schwierig im Rahmen einer Studienplanung eine „Erkältungswelle" zu erfassen, da diese Kranken die Praxis meistens akut aufsuchen. Der vorgegebene zeitliche Rahmen im März und April stellte sich als günstig heraus und ermöglichte die Dokumentation von 273 Patienten.

Die Fragen auf dem entsprechenden Bogen zu Person und Praxis wurden von allen Ärzten beantwortet. Lediglich Angaben zur Größe der Praxis gemessen an der pro Quartal erzielten Scheinzahl wurden von einigen Ärzten nur sehr widerwillig gemacht. 
Bei den Hospitationen herrschte eine durchweg sehr freundliche Atmosphäre sowohl von Seiten der Praxisinhaber als auch von deren ärztlichen Hilfspersonal. Bis auf einen Arzt, der mich nur zu den Patienten mit akuten Atemwegsinfekten in sein Sprechzimmer rief, durfte ich bei allen anderen Konsultationen anwesend sein. Für mich war es allerdings relativ anstrengend mich jeden Tag auf einen anderen Arzt, Räumlichkeiten und Personal einzustellen. Um noch mehr Patienten zu rekrutieren, hätte man die Hospitationen vielleicht auf 2 Tage ausdehnen sollen.

\subsection{Rücklauf der Anschreiben}

Die relativ hohe Rücklaufquote bei der vorliegenden Studie ist möglicherweise auf ein sehr einfaches Antwortverfahren zurückzuführen. Hervorzuheben ist aber auch die Studienbereitschaft der Ärzte im Briloner Raum. Es spielt dabei möglicherweise eine Rolle, dass sie nicht so häufig zur Teilnahme an wissenschaftlichen Studien aufgefordert werden. Außerdem liegt Brilon nicht in unmittelbarer Nähe einer medizinischen Fakultät und die Ärzte haben relativ wenig Kontakt zu universitären Einrichtungen bzw. Studierenden. Wie drückte es ein Briloner Facharzt aus: „Das ist ja schön, dass sich die Uni auch einmal für uns interessiert. Die denken doch sowieso, dass wir Ärzte auf dem Lande schlechtere Medizin machen.“

\subsection{Beschreibung der Stichprobe und des Patientenkollektivs}

Der Anteil der niedergelassenen Fachärztinnen für Allgemeinmedizin an der Gesamtzahl der Ärzte dieser Fachrichtung war in Brilon und Hildesheim, verglichen mit der zum 31. Dezember 1997 erstellten Statistik der Bundesärztekammer (Thust und Lieschke 1999), unterdurchschnittlich. Dort wird bundesweit ein Anteil von 33,7\% niedergelassener Allgemeinmedizinerinnen beschrieben. Dieser betrug in der vorliegenden Studie 16,7\% und entsprach nur der Hälfte der Zahl der niedergelassenen Fachärztinnen bundesweit.

Die Altersverteilung der teilnehmenden Ärzte entsprach der bundesweiten Verteilung 1997 (Thust und Lieschke 1999). Auch hier waren die Altersgruppen der 40-49 und 50-59 Jahre alten Fachärzte für Allgemeinmedizin am häufigsten vertreten. 
Der Anteil der Atemwegsinfekte an den Gesamtkonsultationen am Tag der Hospitation lag bei insgesamt 21,4\% $( \pm 6,4)$ und war damit etwas geringer als der von Monto und Sullivan (1993) beschriebene von 25,4\%. Sie führten eine Beobachtung zur Ätiologie und Epidemiologie von Atemwegsinfekten bei 5363 Personen in Tecumseh, Michigan, über insgesamt 11 Jahre durch.

Schnur und Exner (1997) beschrieben den Anteil akuter respiratorischer Erkrankungen an den Gesamtkonsultationen in einer Sentinel-Praxis in den Jahren 1993 und 1994 für jeden einzelnen Monat. Im Vergleich zu den vorliegenden Daten fanden sie im gewählten Beobachtungszeitraum März und April 1993 einen Prozentsatz von 16,1\% bzw. 5,9\% und für 1994 von 10,5\% bzw. $6,6 \%$, bezogen auf die Gesamtkonsultationen.

Im Hinblick auf die Altersverteilung der erkrankten Personen wies die vorliegende Studie ein Maximum der Konsultationen für die Altersgruppe 30-39 Jahre aus. Die meisten Folgekontakte waren den 70- bis 79-Jährigen zuzuordnen, was mit einem protahierten Krankheitsverlauf erklärt werden könnte. Dies entspricht Daten von Schnur und Exner (1997) zur Häufigkeit von respiratorischen Infektionen in den Jahren 1993 und 1994. Sie haben ein Maximum der Krankheitsaktivität im 3. und 4. Lebensjahrzehnt mit einem Abfall ab der 5. Lebensdekade gefunden.

\subsection{Patientenanamnese}

Bei der Anamnese wiesen die Erkrankten eine Vielzahl von Symptomen auf (siehe Tabelle 4). Insgesamt wurden 25 verschiedene Symptome benannt, wobei die 3 Symptome Husten, Schnupfen, Halsschmerzen sowohl bei Erst- als auch bei Folgekontakten am häufigsten angegeben wurden.

In Deutschland liegt zur Zeit nur eine evidenzbasierte Handlungsleitlinie zur Diagnostik und Therapie von Atemwegsinfekten von der Arzneimittelkommission der deutschen Ärzteschaft (2000) vor. Eine konkrete Anleitung zur Erhebung der Anamnese bei Atemwegsinfekten wird hier jedoch nicht gegeben. Es werden lediglich einige Symptome und Untersuchungsbefunde zur Differentialdiagnose zwischen bakteriellen und viralen Atemwegsinfekten aufgezählt. So sprächen etwa ein akuter Beginn, hohes Fieber, Tonsillenbeläge, Husten mit Auswurf und 
pathologische Befunde bei der Lungenauskultation für einen bakteriellen Infekt. Die DEGAM (Deutsche Gesellschaft für Allgemein- und Familienmedizin) erarbeitet derzeit Leitlinien zu den Symptomen Husten, Halsschmerzen und akute Sinusitis.

Bei der Recherche zu weiteren Leitlinien anderer Länder über die bekannten Datenbanken traten folgende Probleme auf:

- eine Evidenz der Leitlinien fehlt häufig,

- der Zugang zu den Leitlinien ist z.T. nur durch ein Passwort möglich und es ist somit keine freie Verfügbarkeit gegeben

- es fehlen Angaben zur Anamnese von Atemwegsinfekten.

Ferner muss diskutiert werden, ob diese Leitlinien auf Deutschland übertragen werden können (z.B. unterschiedliche Resistenzsituation der Erreger, verschiedene Gesundheitssysteme).

\subsection{Durchgeführte Untersuchungen}

\subsubsection{Körperliche Untersuchung und Untersuchungsbefund}

99,3\% der Patienten wurden körperlich untersucht (alle Erstkontakte und 97,7\% der Folgekontakte). In der Handlungsleitlinie zu akuten Atemwegsinfekten der Arzneimittelkommission der deutschen Ärzteschaft (2000) wird zur Diagnostik die Anamnese und der körperliche Untersuchungsbefund als ausreichend angesehen. Die Art der durchzuführenden körperlichen Untersuchungen wird jedoch nicht weiter präzisiert. Zu diesem Thema gibt es in Deutschland derzeit noch keine evidenzbasierten Leitlinien.

Tabelle 6 zeigt, dass die Auskultation der Lunge und die Inspektion des Mund-Rachenraumes am häufigsten vorgenommen wurden. Hier fanden sich auch die meisten pathologischen Befunde. Offensichtlich war der Stellenwert dieser Untersuchungen für die Teilnehmenden hoch, da die erhobenen Befunde einen starken Einfluss auf die Therapie (z.B. bei der Antibiotikaverordnung) hatten (s. Tabelle 17). Bei einigen Ärzten konnte beobachtet werden, dass die Auskultation der Lunge ohne Entkleidung des Oberkörpers erfolgte („Kassendreieck"). Über eine orientierende 
Untersuchung bzw. symbolische Handlung kann diese Form der Technik wohl nicht hinausgehen.

Die erhobene Anamnese und der körperliche Untersuchungsbefund könnten nach Meinung von Evertz et al. (1999) standardisiert in eine Datenmaske am Computer eingegeben werden. Diese Maske wurde vom hausärztlichen Qualitätszirkel Leverkusen entwickelt. Die nach Meinung der teilnehmenden Ärzte häufig geschilderten Beschwerden wurden gesammelt (Brainstorming) und nach einem Metaplan-gestützten System gewichtet und ausgewählt. Mit der entwickelten Datenmaske für die Anamnese akuter Atemwegsinfekte konnten 90\% der vorstelligen Patienten erfasst werden. Als Vorteile eines solchen Vorgehens sehen die Autoren die schnellere Abrufbarkeit der Befunde bei einer möglichen Folgekonsultation, die Möglichkeit Komplikationen rascher $\mathrm{zu}$ erfassen und eine Vereinfachung und Beschleunigung der Arbeitsabläufe.

\subsubsection{Labor- und apparative Diagnostik}

Bei 6,6\% der Patienten wurden Laboruntersuchungen durchgeführt. Die Bestimmung der Leukozyten bzw. des kleinen Blutbildes standen dabei im Vordergrund. Die Bestimmung des Creaktiven Proteins (CRP) kam nur für 2 Patienten in Betracht.

In der bereits vorgestellten evidenzbasierten Leitlinie der Arzneimittelkommission der deutschen Ärzteschaft (2000) zur Therapie akuter Atemwegsinfekte wird die Bestimmung des CRP zur Abgrenzung bakterieller von viralen Infektionen beschrieben. Es erfolgt jedoch keine Aussage darüber, wann eine Bestimmung dieses Laborwertes sinnvoll ist. Nach internationalen Studien sprechen CRP-Werte oberhalb von $40 \mathrm{mg} / \mathrm{dl}$ für einen bakteriellen Infekt, wenngleich die Spezifität nur begrenzt ist (Korppi und Kröger 1992, Hjortdahl et al. 1991). Für den niedergelassenen Arzt steht ein CRP-Schnelltest zur Verfügung. So könnte bei diagnostischer Unsicherheit, ob es sich um eine bakterielle Infektion handelt, insbesondere im Hinblick auf eine mögliche Antibiotikaverordnung, ein solcher Test durchgeführt werden. 
Bei keinem der dokumentierten Patienten wurde eine mikrobiologische Sputumdiagnostik angeordnet. Dies deckt sich mit den Empfehlungen einer Expertengruppe der Paul-EhrlichGesellschaft für Chemotherapie e.V. und der Deutschen Atemwegsliga e. V., die von Vogel et al. (2000) publiziert wurden. Sie sehen eine Indikation zur mikrobiologischen Diagnostik bei infektiösen Bronchialerkrankungen nur bei einem Nichtansprechen der initialen Therapie innerhalb von 72 Stunden, beim Vorliegen einer schweren Grunderkrankung, bei Häufung akuter Schübe einer Bronchitis und bei Komplikationen.

Es wurde ebenfalls auf Streptokokkenschnelltests und Rachenabstriche verzichtet, obwohl bei 21 Patienten mit Erstkontakt und 9 Patienten mit Folgekontakt eine akute Tonsillitis diagnostiziert wurde. Auf die Bedeutung des Streptokokkenschnelltests für die Allgemeinarztpraxis wird in Kapitel 4.10 .3 eingegangen.

Bei insgesamt 9 Patienten wurde die Lungenfunktion geprüft. Die Leitlinie besagter Arzneimittelkommission der deutschen Ärzteschaft (2000) empfiehlt eine Lungenfunktionsprüfung bei Dyspnoe oder klinischen Hinweisen auf eine Bronchialobstruktion. Diese Kriterien erfüllten 5 der 9 Patienten. Bei den anderen 4 Patienten war die Indikation der Lungenfunktionsprüfung nicht aus den erhobenen Symptomen oder Befunden ableitbar. Die o.g. Leitlinie wurde zwar speziell für den allgemeinärztlich/hausärztlichen Bereich konzipiert; die geforderte Blutgasanalyse zeigt jedoch, wie wenig sie tatsächlich an den hausärztlichen Gegebenheiten orientiert ist, da diese Analyse in der Allgemeinarztpraxis technisch kaum möglich ist.

\subsection{3 Überweisung zu Spezialisten oder ins Krankenhaus}

Es wurden insgesamt 2,6\% der Patienten zu Spezialisten überwiesen. Diese geringe Rate spricht dafür, dass die Fachärzte für Allgemeinmedizin die Behandlung von respiratorischen Infektionen zunächst als ihren Behandlungsauftrag ansehen und unnötige Diagnostik und Therapie vermeiden wollen. Diese Beobachtung deckt sich auch mit einer Studie von de Melker und Kuyvenhoven (1994) nach der 3\% aller Patienten zum HNO-Arzt überwiesen wurden (1\% der Erstkontakte und $6 \%$ der Folgekontakte). 
Bei 2 Patienten hielten die jeweiligen Ärzte eine Röntgenaufnahme des Thorax für indiziert. Der Erste (65 Jahre alt) wies mit 4 Wochen Krankheitsdauer einen protahierten Verlauf auf, die Zweite (72 Jahre) litt seit einer Woche an den Symptomen einer akuten Exazerbation einer chronischen Bronchitis und stellte sich zum 2. Mal vor. Die Handlungsleitlinie der bereits mehrfach zitierten Arzneimittelkommission der deutschen Ärzteschaft (2000) empfiehlt eine Röntgen-Thoraxaufnahme in 2 Ebenen bei Verdacht auf Pneumonie, protrahiertem Krankheitsverlauf und zugrunde liegenden bronchopulmonalen Erkrankungen. Es werden jedoch keine Angaben $\mathrm{zu}$ der Frage gemacht, ab welchem Zeitraum von einem protrahierten Krankheitsverlauf auszugehen ist und welche zugrunde liegenden bronchopulmonalen Erkrankungen gemeint sind. Die beiden in dieser Studie veranlassten Röntgen-ThoraxUntersuchungen sind demnach leitlinienkonform erfolgt.

Eine Einweisung ins Krankenhaus hielten die teilnehmenden Ärzte bei niemandem für erforderlich. Es wurde nur einmal eine Pneumonie diagnostiziert. Bei diesem Patienten war bereits eine Röntgenaufnahme des Thorax erfolgt und dabei ebenfalls der Verdacht auf eine maligne Erkrankung der Lunge geäußert worden. Dieser Patient wurde zur weiteren Abklärung zunächst zu einem niedergelassenen Facharzt für Pulmonologie überwiesen.

\subsection{Diagnosen}

Die Einteilung der Diagnosen erfolgte nach ICPC (International Code of Primary Care), der jedoch den meisten Ärzten nicht bekannt war. Es fiel dabei eine große Bandbreite bei der Nomenklatur der verwendeten Krankheitsdiagnosen auf. So wurde die Diagnose des grippalen Infektes noch relativ häufig verwendet, obwohl sie weder im ICD Version 9 oder 10 noch im ICPC als eigenständige Diagnose Berücksichtigung findet.

Die Häufigkeitsverteilung der 5 im Vordergrund stehenden Diagnosen entspricht in etwa denen einer Studie von Hummers-Pradier et al. (1999) zur Behandlung von Atemwegsinfekten, die in 18 deutschen Allgemeinarztpraxen durchgeführt wurde. Dabei unterschied man jedoch nicht zwischen Erst- und Folgekontakten. In den weiteren Darstellungen wird auf die Diskussion der 
Diagnostik und Therapie der akuten Exazerbationen chronischer Lungenerkrankungen verzichtet, da die Fallzahl von jeweils 12 Patienten bei Erst- oder Folgekontakten zu gering war. Gleiches gilt für die Diagnose der akuten Otitis media.

\subsection{Therapie}

\subsubsection{Art und Häufigkeit der Medikamentenverschreibung}

Über 98\% der Erstkontakte erhielten zumindest die Verordnung eines Medikamentes oder ihnen wurde es als Musterpräparat mitgegeben. 67,2\% der Erst- und 44,4\% der Folgekontakte wurden sogar 2 oder mehr Medikamente rezeptiert. Gerade diese hohe Zahl an Mehrfachverordnungen kann nicht nur durch die in Kapitel 1.6 aufgeführten, das Verordnungsverhalten beeinflussenden Faktoren erklärt werden (Befriedigung der Patientenerwartung, Pseudo-Plazebo-Verordnung etc.).

Sowohl bei Erst- als auch bei Folgekontakten erhielten die meisten Patienten eine oder mehrere Verordnungen aus der Gruppe der Husten- und Erkältungspräparate, 48,7\% auch ein Antibiotikum. Auf die Bedeutung des Einsatzes von Antibiotika und Husten- und Erkältungspräparaten wird gesondert in Kapitel 4.8 und 4.9 eingegangen. Hummers-Pradier et al. (1999) kommen zu ähnlichen Ergebnissen in ihrer Studie zur Therapie von Atemwegsinfekten in 18 deutschen Allgemeinarztpraxen. Hier erhielten 90\% der Patienten eine Verordnung, davon 27,7\% die eines Antibiotikums und 67\% die eines oder mehrerer Präparate aus der Gruppe der Husten- und Erkältungspräparate.

Vergleiche mit Studien anderer Ländern sind auf Grund anderer ökonomischer Bedingungen nur bedingt möglich. So spielt die Verschreibung von Husten- und Erkältungspräparaten in den USA eine untergeordnete Rolle, während die Gabe von Antibiotika im Vordergrund steht (Mainous III et al. 1997). Dies wird in den Ergebnissen einer Fragebogenstudie mit Fallbeschreibungen von Mainous III et al. (1997 a) reflektiert, in der das Patientenwissen zu oberen Atemwegsinfekten untersucht wurde. $46 \%$ der Teilnehmer hielten eine Antibiotikaverordnung bei den Symptomen 
Husten, Halsschmerzen und klares Nasensekret mit einer Symptomdauer von 5 Tagen für indiziert. Wenn purulentes Nasensekret hinzukam, wünschten 69\% ein Antibiotikum.

Legt man $\$ 34$ Abs. 1 SGB V zu Grunde, zählen Arzneimittel zur Anwendung bei Erkältungskrankheiten und grippalen Infekten einschließlich hierbei angewendeter Schnupfenmittel, Schmerzmittel, hustendämpfender und hustenlösender Mittel sowie Mund- und Rachentherapeutika zu den leistungsrechtlich ausgeschlossenen Mitteln (Schwabe 1999 b), da sie üblicherweise bei schwachen gesundheitlichen Beeinträchtigungen eingesetzt werden (sog. Bagatellarzneimittel). Die Abgrenzung relevanter Indikationen bei einigen Arzneimittelgruppen gegenüber geringfügigen Gesundheitsstörungen ist jedoch nicht möglich (z.B. Einsatz von Acetylsalicylsäure bei Kopfschmerzen, Migräneattacken usw.), sodass die Verordnung z.T. gerechtfertigt sein kann.

Zum Zeitpunkt der Datenerhebung im Jahr 1997 betrug der Umsatz leistungsrechtlich ausgeschlossener Mittel insgesamt 823,5 Millionen DM bei 60 Millionen Verordnungen (Schwabe 1999 b). Bezogen auf die vorliegende Studie gehören 70,1\% der verordneten Arzneimittel (Rhinologika, Halsschmerzmittel, Brusteinreibungen und sonstige Inhalate, Hustenund Erkältungspräparate, Echinacea-Zubereitungen) zu dieser Gruppe. Hieraus ergeben sich demnach erhebliche Einsparungspotentiale.

\subsubsection{Verschreibungspraxis}

2,9\% der Patienten baten den Arzt um die Verschreibung eines bestimmten Präparates. Wie schon in Kapitel 1.6 dargestellt wird der vermeintliche Arzneimittelwunsch möglicherweise von den Ärzten überinterpretiert oder die Patienten wagen nicht ihre Wünsche zu artikulieren. Eine Lösung dieser Problematik könnte darin bestehen, den Patienten konkret zu fragen, ob er die Verordnung eines Medikamentes wünscht, wie es Himmel et al. (1997) vorschlagen.

Der Wunsch bezüglich eines Antibiotikums wurde nur von einem Patienten geäußert. Dies erscheint umso erstaunlicher, wenn man Untersuchungen zu Patientenerwartungen hinsichtlich einer möglichen Antibiotikaverordnung bei Atemwegsinfekten betrachtet. Hamm RM et al. 
(1996) verteilten dazu einen Fragebogen an insgesamt 113 Patienten. Vor der Konsultation erwarteten 65\% eine Antibiotikaverordnung, 63\% erhielten auch ein Rezept. Wenn die Ärzte der Überzeugung waren, die Patienten wünschten ein Antibiotikum, erhielten $77 \%$ eine Verschreibung. Wenn sie jedoch zu der Ansicht gelangten, dass sie keine Verordnung wünschten, wurde nur 29\% der Patienten ein Antibiotikum verschrieben. Bei Unsicherheit hinsichtlich der Patientenerwartungen erhielten $72 \%$ ein Antibiotikum. Die Autoren konnten 3 Faktoren zeigen, die Ärzte in ihrer Entscheidung beeinflussten sich für ein Antibiotikum zu entscheiden: die gestellte Diagnose, die Überzeugung, dass Antibiotika in diesem Fall indiziert waren und die Überzeugung, dass Patienten eine solche Verordnung wünschten. Keine Assoziation konnte zwischen der Antibiotikaverschreibung und der Patientenzufriedenheit dargestellt werden. Letztere korrelierte am stärksten damit, ob der Arzt sich genügend Zeit für die Erklärung des natürlichen Verlaufs der Erkrankung und ihrer Therapie genommen hatte und die Patienten diese Erklärungen auch verstanden hatten. Basierend auf diesen Ergebnissen könnten unnötige Antibiotikaverschreibungen durch verbesserte Aufklärung vermieden werden, worauf in Kapitel 4.8.3 noch näher eingegangen wird.

Die Empfehlung zum Selbstkauf von Medikamenten stellte eher eine Ausnahme dar. Nur 2,6\% der Patienten erhielten eine derartige Empfehlung. Besonders vor dem Hintergrund, dass die meisten Husten- und Erkältungspräparate frei verkäuflich sind und zudem als sogenannte Bagatellarzneimittel nicht verordnet werden dürfen, ist diese Vorgehensweise diskussionswürdig.

Alle 3 am häufigsten verordneten Medikamente Acetylcystein, Ambroxol und Paracetamol sind frei verkäuflich. Bei den im Untersuchungszeitraum gültigen Selbstbeteiligungen von 4 DM (N1), 6 DM (N2) und 8 DM (N3) unterschieden sich diese und die Preise im freien Verkauf nur unwesentlich. So kostete z.B. eine N2 Schachtel mit 30 Tabletten Paracetamol der Firma Stada 3,15 DM (Rote Liste 1997). Die Zuzahlungskosten überstiegen demnach sogar den Preis des Medikamentes. Ähnlich sieht es mit den Preisen für Acetylcystein aus. Eine N1-Schachtel mit 20 Brausetabletten desselben Herstellers kostete 9,35 DM (Rote Liste 1997). 


\subsubsection{Ausstellung einer Arbeitsunfähigkeitsbescheinigung (AU), Wiedereinbestellung und Rekonsultation}

Erkrankungen der Atemwege gehören zu den häufigsten Gründen einer Krankschreibung. In einer Untersuchung von Himmel et. al. (1995) waren 43,2\% der Arbeitsunfähigkeitsbescheinigungen auf diese Problematik zurückzuführen. Die Rate der Krankschreibungen lag in den 14 teilnehmenden Allgemeinarztpraxen bei insgesamt 40\% der für eine Arbeitsunfähigkeitsbescheinigung in Frage kommenden Patientenkontakte. Die einzelnen Praxen unterschieden sich mit Raten zwischen 13\% und 60\%. 6\% der Patienten erhielten eine erneute Ausfertigung.

Die Zahl der Krankschreibungen lag in der vorliegenden Arbeit höher, wobei hier nur die Erwerbstätigen berücksichtigt wurden. 56,8\% erhielten eine Arbeitsunfähigkeitsbescheinigung, fast 20\% der Erwerbstätigen lehnten sie ab. Der Verzicht entspricht dem allgemeinen Trend, dass die Krankheitstage im Vergleich $\mathrm{zu}$ den Vorjahren zurückgingen (Bundesverband der Betriebskrankenkassen 2002).

$\mathrm{Ob}$ in den in dieser Studie dokumentierten Fällen eine Arbeitsunfähigkeitsbescheinigung wirklich erforderlich war, war nicht Ziel dieser Untersuchung und kann auf Grund der Datenlage nicht beurteilt werden. Auffällig war jedoch der hohe Anteil von Krankschreibungen bei Folgekontakten. 60\% der Erwerbstätigen wurden erstmals oder erneut krank geschrieben,. Eine Folgekonsultation war demnach vielfach mit einer weiteren Krankschreibung verbunden (Dauer $4,1( \pm 1,6)$ Tage $)$.

In der vorliegenden Studie war die Summe der Wiedereinbestellungen hoch. 42,5\% der Patienten wurden gebeten sich wieder vorzustellen. 22,0\% erhielten die Anweisung bei einer Verschlechterung des Zustandes erneut einen Termin zu vereinbaren. Hinsichtlich der gestellten Diagnosen hielten Ärzte Patienten mit der Diagnose einer akuten Bronchitis $(61,4 \%)$ bzw. einer akuten Tonsillitis $(63,3 \%)$ für besonders kontrollbedürftig und empfahlen eine neuerliche Vorstellung. Immerhin sollten aber auch 23,1\% mit einem oberen Atemwegsinfekt nochmals die Praxis aufsuchen, was auf Grund des natürlichen Verlaufes dieser Erkrankung nicht evidenzbasiert ist. Ziel weiterer Untersuchungen könnte sein der Frage nachzugehen, ob sich 
diese Patienten tatsächlich erneut vorstellen und aus welchen Gründen Ärzte Personen mit Bagatellerkrankungen erneut einbestellen.

Ein positiver Zusammenhang zwischen Antibiotikaverordnung und Wiedereinbestellung konnte nachgewiesen werden. $71,8 \%$ der Patienten, die wiederkommen sollten, erhielten ein Antibiotikum. Mit 25\% lag der Anteil dieser Verordnungen bei denen, die nicht noch einmal erscheinen sollten, deutlich niedriger. Diejenigen, die angewiesen wurden sich nur bei Beschwerdepersistenz erneut vorzustellen, erhielten zu 20,8\% eine Antibiotikaverordnung. Die Unterschiede waren im Mehrfeldertest signifikant $(p<0,0001)$. Offensichtlich wollten Ärzte die Wirksamkeit der antibiotischen Therapie kontrollieren oder hielten bei den Patienten auf Grund der Schwere ihres Krankheitsbildes eine Überwachung für indiziert.

Wie 2 Studien von Davey et al. (1994) und Hueston et al. (2000 b) zeigten, konnte die Anzahl der Folgekonsultationen durch die Verordnung von Antibiotika bei akuten Atemwegsinfekten nicht reduziert werden. Davey et al. (1994) untersuchten diese Häufigkeit in einer Praxis während eines Winters. Sie dokumentierten 1140 Patienten mit den Symptomen eines Atemwegsinfektes, von denen 79\% ein Antibiotikum erhielten. Die Rate derartiger Verordnungen lag somit noch höher als in der vorliegenden Studie (60,0\%). Die Autoren kommen zu dem Schluss, dass die Gabe von Antibiotika neben anderen negativen Auswirkungen nicht dazu geeignet war die Zahl der Folgekonsultationen zu reduzieren.

Hueston et al. $(2000$ b) untersuchten in einer retrospektiven Studie von 29248 Krankheitsepisoden von 24753 Patienten mit der Diagnose einer akuten Bronchitis die Häufigkeit von Folgekonsultationen im Hinblick auf die Therapie mit Antibiotika oder Bronchodilatatoren. Die initiale Verschreibung von Antibiotika reduzierte die Häufigkeit von frühen Folgekonsultationen ( $<14$ Tagen) bei Patienten unter 18 Jahren, hatte jedoch keinen Effekt bei Erwachsenen. Allerdings zeigte sich auch nur eine geringe Wirkung bei Patienten unter 18 Jahren. So mussten umgerechnet 33 Patienten ein Antibiotikum erhalten um statistisch einen Folgekontakt zu verhindern. Alten Patienten ( $>65$ Jahre) wurde geringfügig weniger häufig ein Antibiotikum verordnet (59\%) als Patienten unter 18 Jahren (65\%). Die Daten der hier 
vorgestellten Studie weisen keine Abhängigkeit der Antibiotikaverschreibung vom Alter der Patienten auf.

\subsubsection{Selbstmedikation und vom Patienten durchgeführte nichtmedikamentöse Therapie}

17,9\% der Patienten machten Angaben zur Selbstmedikation oder wurden von den Ärzten diesbezüglich befragt. Von diesen gaben $83,7 \%$ an eine Selbstmedikation durchgeführt zu haben.

In einer Studie von Klein (2000) bei 317 Patienten mit neu aufgetretenen Symptomen einer akuten respiratorischen Erkrankung hatten bereits $74 \%$ vor der Erstkonsultation eine Selbstmedikation oder nichtmedikamentöse Therapie durchgeführt. Diese Zahlen belegen den Stellenwert dieser Art von Behandlung. Ein Großteil der hierzu befragten Patienten mit Atemwegsinfekten hatte demnach schon versucht gegen die Beschwerden durch selbst ausgesuchte Arzneimittel vorzugehen. Ärztlicherseits wird hierauf jedoch nur wenig eingegangen, wie die mit 17,9\% nur geringe Quote an Nachfragen belegt.

In einer Fragebogenstudie von Bradley et al. (1998) mit 2765 Patienten in 6 Praxen meinten 52\% der Patienten, dass Ärzte sich routinemäßig nach der Selbstmedikation erkundigen sollten; 30\% waren unentschlossen und 2\% lehnten diese Frage ab. Die Bereitschaft der Patienten Auskunft über die von ihnen eingenommene Selbstmedikation zu geben ist also vorhanden.

Deren Vorteile liegen aus ökonomischer Sicht im Einspareffekt im Sinne der Entlastung der Solidarversicherung und der Steigerung der Eigenverantwortung des Patienten (Himmel 2000). Himmel sieht die Möglichkeit bei Patienten durch das selbst erlernte und selbst erlebte Wissen um die Beeinflussbarkeit von Symptomen eine „Selbstmedikationskultur" zu fördern. Ärzte sollten seiner Meinung nach mit ihren Patienten offen über dieses Thema sprechen, ihnen entsprechende Informationen darüber geben und ein solches Vorgehen nicht als Entmachtung empfinden. Patienten sehen in der Selbstmedikation eine Möglichkeit sich einen Arztbesuch zu ersparen und schnellere Hilfe dadurch zu erhalten, dass sie gleich in die Apotheke gehen können (Walluf-Blume 1997). Außerdem denken sie, dass sie in Zukunft sowieso alle Arzneimittel für leichtere Erkrankungen selbst bezahlen müssen. 
Kochen (2000) nennt Gefahren der Selbstmedikation:

- falsche Selbstdiagnose,

- fehlende Eignung des Präparates,

- Verzögerung notwendiger Therapie,

- Beibehaltung schädlicher Verhaltensweisen,

- Unverträglichkeit mit anderen Arzneimitteln.

Er kritisiert auch, dass es in Deutschland keine Liste von frei verkäuflichen Arzneimitteln gibt, die kostengünstiger sind als die entsprechende Rezeptgebühr. Weiterhin verweist er auf den zumeist fehlenden Wirksamkeitsnachweis der üblichen Produkte.

1998 wurden auf dem Arzneimittelmarkt über 50 Mrd. DM umgesetzt, davon 20\% für die Selbstmedikation (Himmel 2000). Husten- und Erkältungspräparate machten dabei mit 1,54 Mrd. DM) den stärksten Umsatz im Zusammenhang mit der Selbstmedikation aus. Auch in der vorliegenden Studie wurden diese Präparate mit 34 Medikationen am häufigsten angewendet. Bei 39\% der Patienten mit Selbstmedikation waren die Ärzte offensichtlich mit dieser gewählten Medikation einverstanden und rezeptierten ein Medikament aus derselben therapeutischen Hauptgruppe. Bei den Übrigen kann jedoch auf Grund des Studienaufbaus keine Aussage über das Vertrauen der Ärzte in die von den Patienten gewählte Selbstmedikation getroffen werden.

Bei der Selbstmedikation zeigte sich ein Trend zu sogenannten Naturheilmitteln (Flintrop 2002). Flintrop (2002) stellt Studien des Allensbacher Institutes für Demoskopie im Auftrag des Bundesfachverbandes der Arzneimittelhersteller vor. Etwa 60\% der Befragten hatten sich im Jahr 2002 Naturheilmittel selbst gekauft, 1997 waren es noch 56\% der Befragten. 69\% glaubten an die Wirksamkeit von Naturheilmitteln, insbesondere bei Erkältungen (Flintrop 2002). In der vorliegenden Studie betrug der Anteil der verschriebenen pflanzlichen Arzneiformen an der Gesamtzahl der Verordnungen 12,9\% und war somit geringer als bei den Untersuchungen des Allensbacher Institutes, nach denen 1997 und 2002 22\% der Befragten ein Naturheilmittel verschrieben worden war. 
12,1\% der Patienten machten Angaben zur nichtmedikamentösen Therapie oder wurden von den Ärzten dazu befragt. Am häufigsten wurde inhaliert oder gegurgelt. 34,8\% der Patienten erhielten zusätzlich zur medikamentösen Therapie Empfehlungen zur nichtmedikamentösen Therapie, wobei Hinweise auf eine erhöhte Flüssigkeitszufuhr und die Durchführung von Inhalationen im Vordergrund standen. Es gibt leider nur wenige Studien zur Wirksamkeit nichtmedikamentöser Therapie bei Atemwegsinfekten. Die Empfehlungen beruhen wahrscheinlich eher auf eigenen Erfahrungen, solchen aus Familie und Bekanntenkreis oder auf Informationen aus der Laienpresse.

Die Effektivität von Inhalationen mit heißem Wasserdampf konnte in 2 Studien von Macknin et al. (1990) und Hendley et al. (1994) im Bezug auf die Reduktion der Symptome, in der manometrischen Untersuchung und dem subjektiven Befinden der Patienten nicht nachgewiesen werden.

Die Wirksamkeit erhöhter Flüssigkeitszufuhr wurde von Shim et al. (1987) bei Patienten mit chronischer Bronchitis untersucht. Es zeigte sich dabei kein Effekt bezüglich der Menge und Elastizität des Sputums, die angegebene Symptomatik und die FEV 1 (Einsekundenkapazität). Studien zur Effektivität erhöhter Flüssigkeitszufuhr bei akuten Atemwegsinfekten liegen nicht vor, sodass keine evidenzbasierten Empfehlungen gegeben werden können. Immerhin wurden jedoch in der vorliegenden Studie 52 Patienten $(18,2 \%)$ auf die Notwendigkeit einer gesteigerten Flüssigkeitszufuhr aufmerksam gemacht.

Der Beipackzettel der Medikamente mit dem Wirkstoff Acetylcystein enthält als zusätzlichen Hinweis, dass die schleimlösende Wirkung durch Flüssigkeitszufuhr erhöht wird. 27,0\% der Patienten, denen ein Medikament mit diesem Wirkstoff verordnet wurde, erhielten auch den Rat mehr zu trinken. Es gibt allerdings auch hier keine Studien, ob die vermeintliche Wirksamkeit des Medikamentes durch die erhöhte Flüssigkeitszufuhr oder durch den Wirkstoff bedingt ist. Darauf wird näher in Kapitel 4.9 eingegangen. 
In Bezug auf das Rauchen scheinen die an der Studie teilnehmenden Ärzte müde zu sein ständig den moralischen Zeigefinger zu heben und ihren Patienten das Rauchen zu verbieten. Nur 27,1\% der Raucher wurde empfohlen, das Rauchen einzustellen.

Wie in einer der besuchten Praxen eingeführt, stellt die Weitergabe von sogenannten „Infozepten“ bei Atemwegsinfekten eine interessante Alternative zur Medikamentenverordnung dar. Lichte (1999) et al. beschreiben eine standardisierte, werbungsfreie, in Rezeptform verfasste Patienteninformation, die individuell ergänzt und vervielfältigt werden kann. Er nennt die Vorteile von „Infozepten“:

- Verbesserung der Arzt-Patient-Beziehung durch effektive Kommunikation: Ergänzung und Verstärkung der mündlichen Kommunikationsinhalte,

- strukturiertere Beratung des Patienten durch den Hausarzt,

- Vorbeugung einer Enttäuschung die Praxis mit leeren Händen, d.h. ohne Rezept, zu verlassen

- Ersatz der Rezeptierung von Bagatell-Arzneimitteln,

- Förderung der Patientenmitverantwortung,

- Organisationshilfe für den Praxisablauf.

Auch die Kassenärztliche Vereinigung Niedersachsens (KVN) und die niedersächsischen Krankenkassen haben als KVN-Rezept-Info Nr.4 eine kopierfähige Patienteninformation entworfen, die den Vertragsärzten bei der Diskussion mit an einem grippalen Infekt Erkrankten helfen soll (Lichte 2001). Durch diese „offizielle Information" der Vertragsärzte und Krankenkassen können eventuelle Diskussionen über die Verordnung von Arzneimitteln, die nach $\S 34$ SGB V von der Verordnung ausgeschlossen sind, vermieden werden und Kosten eingespart werden.

Trotz der fehlenden Evidenz sogenannter „Hausmittel” werden von der Arzneimittelkommission der deutschen Ärzteschaft (2000) Empfehlungen zur nichtmedikamentösen Therapie (Einstellung des Rauchens, ausreichende Flüssigkeitszufuhr, Bettruhe bei Fieber, Anwendung physikalischer Maßnahmen wie z.B. Dampfinhalationen, Hals-, Brust- und Wadenwickel und lokale Wärme) bei akuten Atemwegsinfekten ausgesprochen. Des Weiteren werden symptomatische Medikamente 
genannt, deren Wirksamkeit nicht ausreichend gesichert ist (Vitamin C, echinaceahaltige Präparate, Zink, Sekretolytika, Antihistaminika, Lokaltherapeutika, ätherische Öle).

\subsection{Verordnung von Antibiotika bei Atemwegsinfekten}

Im Zusammenhang mit einer antibiotischen Therapie wird von Experten oft der Satz gebraucht: „Je öfter man die antibiotischen Waffen benutzt, umso stumpfer werden sie" (Tauchnitz 2000). In der vorliegenden Studie wurden diese „Waffen" sehr häufig eingesetzt. Bezogen auf die Gesamtkonsultationen erhielten 133 der 273 Patienten (48,7\%) mit den Symptomen eines Atemwegsinfektes beim Erst- oder Folgekontakt ein Antibiotikum. Vor dem Hintergrund, dass 95\% aller akuten Infektionen des oberen Respirationstraktes durch Viren verursacht werden (Adam et al. 1991) und somit einer antibakteriellen Therapie nicht zugängig sind, muss dieses Verordnungsverhalten kritisiert werden. Vor dem Einsatz von Antibiotika ist eine kritische Indikationsstellung notwendig. Diese sollte das Risiko von Nebenwirkungen und die Gefahr von Resistenzentwicklungen berücksichtigen (Tauchnitz 2000). Auf die Verordnung von Antibiotika bei den 4 häufigsten Diagnosen der vorliegenden Erhebung wird in den entsprechenden Kapiteln zur Diagnostik und Therapie dieser Krankheitsbilder eingegangen (s. Kapitel 4.10).

\subsubsection{Art der verschriebenen Antibiotika}

Wie in Kapitel 3.7.1 dargestellt wurde die pharmakologische Hauptgruppe der Makrolide und Lincosamide insgesamt am meisten verordnet (37,5\%). An 2. Stelle folgten Betalactamantibiotika und Penicilline (21,5\%). Doxycyclin als Monosubstanz oder in Kombination mit Expektoranzien (20,8\%) stand an 3. Stelle.

Bundesweit waren zum Zeitpunkt der Datenerhebung 1997 jedoch Tetracycline (Schmitz 1999) mit 66,4 Millionen DDD (defined daily dose) die am häufigsten verordneten Antibiotika. Dabei machten Doxycyclinpräparate mit über 90\% (62,8 Millionen DDD) den größten Anteil aus. Hier ist die besondere Kostengünstigkeit der Präparate mit 0,70 DM DDD-Kosten zu erwähnen, was sicherlich einen Einfluss auf deren Beliebtheit hatte. Makrolidantibiotika und Clindamycin fanden sich mit 60,2 Millionen DDD an 2. Stelle. Im Jahr 2001 setzte sich der seit 1993 
rückläufige Verordnungstrend von Tetracyclinen weiter fort (Schwabe 2002 b). Es wurden 12,3\% weniger Doxycyclinpräparate verordnet (54,7 Millionen DDD). Makrolidantibiotika und Clindamycin waren nach den Aminopenicillinen (65,7 Millionen DDD) die Antibiotikagruppe, die am dritthäufigsten verschrieben wurde (57,2 Millionen DDD).

Die 3 beliebtesten Antibiotika 1997 stammten ohne Ausnahme aus der Gruppe der Makrolidantibiotika. Dabei sind vor allen Dingen die relativ hohen DDD-Kosten gegenüber anderen Antibiotika erwähnenswert $\left(\right.$ Rulid $^{\circledR}$ 7,11 DM; Klacid ${ }^{\circledR}$ 10,76 DM; Zithromax ${ }^{\circledR}$ 10,02 DM). 1997 war Rulid $^{\circledR}$ (chemische Substanz Roxithromycin) mit 25,16 Millionen Verordnungen und einem Umsatz von 122,3 Millionen DM das Antibiotikum, das am meisten verschrieben wurde (Schmitz 1999). Auch in der vorliegenden Studie wurde Roxithromycin als Monosubstanz

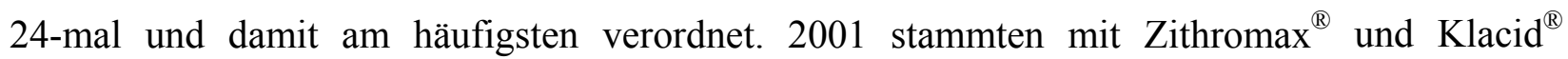
ebenfalls die 2 verordnungshäufigsten Präparate aus der Gruppe der Makrolide.

Die Expertengruppe der Paul-Ehrlich-Gesellschaft für Chemotherapie e.V. und der Deutschen Atemwegsliga e.V. beschreiben in ihren Empfehlungen zur rationellen Therapie bakterieller Atemwegsinfektionen (Vogel et al. 2000) jedoch einen Anstieg makrolid-resistenter Pneumokokken (bis zu 14\%) und Streptokokken der Gruppe A. Tauchnitz (2000) sieht die Ursache dafür in dem erheblichen Verbrauchsanstieg, der seiner Meinung nach durch die AntiDoxycyclin-Kampagnen der letzten 10 Jahre ausgelöst wurde und zu einem Zuwachs der Verordnungen von teureren Oral-Cephalosporinen und Makroliden führte. Dies war mit angeblich hohen Resistenzraten von bis zu 80\% von Doxycyclin gegenüber den wichtigsten Erregern von Atemwegsinfekten begründet worden. Dieser Wert, so Tauchnitz (2000), sei jedoch völlig aus der Luft gegriffen. Die Doxycyclinresistenz von Pneumokokken liegt nach Untersuchungen von Kresken et al. (2000) zur überregionalen Resistenzlage nur halb so hoch wie die ständig wachsende Makrolidresistenz. Dabei handelt es sich allerdings um Daten, die 1998 aus klinischen Isolaten gewonnen wurden. Die Expertengruppe der Paul-Ehrlich-Gesellschaft für Chemotherapie e.V. und der Deutschen Atemwegsliga e.V. (Vogel et al. 2000) halten Doxycyclin allerdings nur dann für eine empirischen Initialtherapie geeignet, wenn die lokale Resistenzlage hinreichend bekannt ist. 


\subsubsection{Prädiktoren für eine antibiotische Therapie}

Eine Zielsetzung der vorliegenden Arbeit war die Ermittlung von Prädiktoren für eine antibiotische Therapie. Wie in den Kapiteln 3.7.3 und 3.7.4 dargestellt fanden sich nur wenige Symptome und Faktoren, die signifikant häufiger mit einer Antibiotiakaverordnung assoziiert waren.

So wurden gelblich-grüner Auswurf und das Vorhandensein von Stippchen und Belägen auf den Tonsillen von den Ärzten als Zeichen einer bakteriellen Infektion gewertet, die eine antibiotische Therapie notwendig erschienen ließen. Dies entspricht den Ergebnissen von Mainous III et al. (1997 ), die in einer Fallvignetten-Studie mit US-amerikanischen Allgemeinärzten ebenfalls die Farbe des Sputums als Prädiktor einer Antibiotikaverordnung nachweisen konnten. Auch Murray et al. (2000) konnten in einer Fallvignetten-Studie mit australischen Allgemeinärzten gelblichen Auswurf als Prädiktor zeigen. Dass die Verfärbung des Sputums jedoch keineswegs Ausdruck einer bakteriellen Infektion sein muss, zeigten Winther et al. (1984). Lediglich bei chronischer Bronchitis gibt es Hinweise, dass gelblich-grüner Auswurf ein Zeichen einer bakteriellen Infektion ist (Stockley et al. 2000).

Weiterhin wurden Patienten mit den Symptomen Fieber und Abgeschlagenheit vermehrt mit Antibiotika behandelt. Diese Faktoren werden von den teilnehmenden Ärzten offensichtlich als Parameter der Krankheitsschwere angesehen. Das Vorhandensein von Fieber konnte in mehreren Studien zu Atemwegsinfekten durchaus als eine kennzeichnende Größe einer bakteriellen Infektion nachgewiesen werden (Centor et al. 1981, Meyer et al. 2002).

Pathologische Befunde bei der Palpation der Nasennebenhöhlen, der Auskultation der Lungen und der Otoskopie waren ebenfalls Prädiktoren der Antibiotikaverordnung. Dies steht in Einklang mit Ergebnissen von Dosh et al. (2000), die in Michigan bei 482 Patienten mit Symptomen eines respiratorischen Infektes die Einflussfaktoren einer Antibiotikaverordnung untersuchten. Auch bei einer Studie von Gonzales et al. (1999) an 322 Patienten in drei allgemeinärztlichen Praxen in Denver konnten u.a. druckschmerzhafte Nasennebenhöhlen als signifikanter Parameter dieser Therapie ermittelt werden. 
Dass ältere Ärzte ( $>50$ Jahre) vermehrt Antibiotika verordneten, könnte mit einem Gewohnheitsverhalten erklärt werden. Möglicherweise hat auch die individuelle ärztliche Erfahrung zu diesem Verhalten geführt. In der Literatur finden sich keine Hinweise für die Abhängigkeit dieses Handelns von dem Alter des Arztes. Allerdings konnten Watkins et al. (2003) in einer Studie zum Verordnungsverhalten britischer Hausärzte zeigen, dass diejenigen, die länger als 20 Jahre niedergelassen waren im Vergleich zu Kollegen, die erst seit einem kürzeren Zeitraum eine Praxis betrieben, höhere Verordnungskosten aufwiesen (OR 4,31). Die hier präsentierten Daten ließen eine derartige Assoziation nicht zu.

Weiterhin verordneten Ärzte aus Gemeinschaftspraxen insgesamt weniger Antibiotika als Einzelpraxisinhaber. Dies steht in Einklang mit der bereits zitierten Studie von Watkins et al. (2003), die zeigen konnte, dass Einzelpraxen im Gegensatz zu Gemeinschaftspraxen höhere Verordnungskosten aufwiesen.

Zusammenfassend kann festgestellt werden, dass die hier ermittelten Prädiktoren der Antibiotikaverordnung gut mit den bisherigen Literaturdaten übereinstimmen. Lediglich der in einer Studie von Gonzales et al. (1999) nachgewiesene Einfluss des Rauchens konnte hier nicht gezeigt werden

Bei der Antibiotikaverschreibung spielen jedoch auch nicht-pharmakologische Faktoren eine Rolle. Avorn und Solomon (2000) fassen Gründe für die nicht indizierte Verschreibung dieser Medikamente zusammen. Aus Sicht des Patienten sind dies:

- Wunsch einer Verordnung,

- Wunsch nach Legitimation der Krankenrolle, Unterstreichung der Schwere der Erkrankung durch die Verabreichung eines Antibiotikums,

- Unwissenheit über die Unwirksamkeit von Antibiotika bei viralen Infektionen,

- Kampagnen der Pharmaunternehmen, insbesondere bei der Einführung neuer, teurerer Medikamente.

Aus Sicht der Ärzte wird genannt:

- Gewährleistung der Patientenzufriedenheit,

- fachliche Defizite, 
- wirtschaftliche Gründe: hoher Patientendurchsatz als Mittel zu gesteigertem Gesamtumsatz,

- schnelle Beendigung einer Konsultation,

- Werbekampagnen der Pharmaunternehmen, speziell zur Einführung neuer, zumeist teurerer Produkte.

Zum Wissenstand von Antibiotikaverordnungen bei akuten respiratorischen Infektionen führten Wilson et al. (1999) eine telefonische Befragung bei 386 Personen durch. 70\% der Teilnehmer hielten diese Mittel bei bakteriellen respiratorischen Infektionen und 55\% bei viralen Infektionen für effektiv. Nur $21 \%$ der befragten Teilnehmer wussten, dass sie nur für bakterielle und nicht für virale Infektionen indiziert sind. Diese Ergebnisse zeigen, wie wichtig es ist den Patienten über die Ätiologie seines Krankheitsbildes und den fehlenden Nutzen einer antibiotischen Therapie zu informieren.

\subsubsection{Bewältigungsstrategien zur Vermeidung unnötiger Antibiotikaverordnungen}

Verschiedene Studien beschäftigen sich mit Konzepten zur Senkung unnötiger Antibiotikaverordnungen bei Atemwegsinfekten. So verordneten Cox und Jones (2001) Antibiotika lediglich beim Vorliegen komplizierender Faktoren (z.B. Immunsuppression). In anderen Fällen erhielten die Patienten ein Merkblatt mit Informationen zur symptomatischen Therapie. Der Antibiotikaverbrauch sank von 56\% auf 19\% nach der Implementierung des neuen Behandlungsprotokolls. Es zeigten sich keine Auswirkungen auf die Zufriedenheit der Patienten, die Krankheitsdauer und die Anzahl der Rekonsultationen. Die Patienten fühlten sich durch das Merkblatt sogar besser informiert.

Eine andere Strategie zur Senkung des zu häufigen Gebrauches von Antibiotika entwickelten Couchman et al. (2000) bei akuten Atemwegsinfekten. Eine Patientengruppe erhielt keine Verordnung eines Antibiotikums, einer anderen Gruppe wurde ein Rezept mit der Anweisung ausgehändigt es nur einzulösen, wenn sich der Zustand verschlechtert oder nicht innerhalb eines individuell vom behandelnden Arzt festgelegten Zeitraumes verbessert. Einer weiteren Gruppe wurde ein Antibiotikarezept mit der Anweisung gegeben es sofort in der Apotheke einzulösen. Die Zufriedenheit der Patienten mit der „Verordnung auf Reserve" lag bei 96,1\% und wurde von 
$50,2 \%$ der Patienten eingelöst. Somit konnte der Antibiotikaverbrauch um insgesamt $15 \%$ gesenkt werden, was die Autoren als wirksame Einsparmöglichkeit ansehen.

Bei der antibiotischen Therapie von Infektionskrankheiten sollte auch die Resistenzentwicklung von Bakterien zu berücksichtigt werden. Eine mögliche Strategie zur Senkung von Antibiotikaresistenzen beschreiben Seppäla et al. (1997) in Finnland, wo zu Beginn der 90erJahre eine zunehmende Resistenz von Streptokokken der Gruppe A gegen Erythromycin zu beobachten war. Eine landesweite Kampagne mit dem Ziel der verringerten Anwendung von Makroliden und der Information über die Möglichkeit des Einsatzes alternativer Antibiotika bzw. des Verzichts auf Antibiotika bei Virusinfektionen führte zu einem signifikanten Rückgang Erythromycin-resistenter Streptokokken der Gruppe A. Die Untersuchung zeigte, dass Informationskampagnen offenbar das Verordnungsverhalten von Ärzten beeinflussen können und die Häufigkeit von Antibiotikaresistenzen durch eine Änderung des Therapieregimes zu senken ist (von 16,4\% im Jahr 1992 auf 8,6\% im Jahr 1996).

Darüber hinaus empfehlen Avorn und Solomon (2000) Computerprogramme mit Algorithmen, die es Ärzten ermöglichen, ihre Indikation zu Antibiotikatherapie zu überprüfen und ggf. die effektivste Therapie herauszufinden. Dieser Erfolg könnte nach ihrer Meinung aber auch durch die schriftliche Veröffentlichung derartiger Algorithmen erzielt werden. Sie beschreiben eine besondere Form der Arztschulung, bei der Ärzte in einer 5-20 Minuten dauernden 1:1-Schulung (,academic detailling") von einem Pharmakologen oder Arzt über das evidenzbasierte Vorgehen bei der Verordnung von Antibiotika informiert werden. Sie überprüften den Effekt einer derartigen Schulung in einer randomisierten Kontrollstudie bei 435 Ärzten in 5 Staaten der USA mit dem Ziel der Reduzierung der Verordnung von bestimmten Medikamenten wie z.B. Cephalosporinen. Von den geschulten Ärzten wurden diese Medikamente zu 14\% weniger verordnet.

Es kann aber festgestellt werden, dass es allerdings schon genügte, wenn Ärzte den von der Arzneimittelkommission der deutschen Ärzteschaft (2000) aufgestellten Leitsatz berücksichtigten, dass unkomplizierte Infekte der oberen Atemwege, darunter auch die akute Virusbronchitis, auf Grund ihrer Ätiologie keiner antibiotischen Therapie bedürfen. 


\subsection{Verordnung von Husten- und Erkältungspräparaten}

Anknüpfend an Kapitel 1.5 gehören Husten- und Erkältungspräparate zu den in Deutschland am häufigsten verordneten Präparaten. Im untersuchten Patientenkollektiv erhielten 87,1\% der Erstkontakte eines aus dieser Gruppe. Expektoranzien stellten dabei den höchsten Anteil mit 58,0\% dar. 53,6\% der Patienten erhielten Acetylcystein. In Deutschland lag der Anteil dieser Substanz 1997 bei fast 70\% (64\% im Jahr 2001) der Verordnungen aus der Gruppe der Antitussiva und Expektoranzien (Lemmer 1999 und 2002). Die Verordnungen dieser Medikamente sind jedoch in den letzten 10 Jahren um ca. 50\% zurückgegangen; dadurch konnten seit 1992356 Millionen Euro eingespart werden (Lemmer 2002).

Nach wie vor liegt für die Verordnung von Expektoranzien bei Atemwegsinfekten kein überzeugender Nachweis der Wirksamkeit vor. Als Beleg für deren Effektivität wird oft die Senkung von akuten Exazerbationen bei chronischer Bronchitis angegeben, wie sie in der aktuellen Metaanalyse der Cochrane Collaboration (Poole und Black 2003) bestätigt wird. Die Autoren fanden dabei auf der Basis von 23 kontrollierten Studien bei einer Langzeitbehandlung (>3 Monate) eine geringe, aber signifikante Reduktion akuter Exazerbationen (-0,07 Exazerbationen pro Patient/ Monat) und eine signifikante Verringerung der Arbeitsunfähigkeit (-0,56 Tage pro Patient/ Monat).

Für die Therapie akuter Infektionen gibt es hingegen kaum eine Evidenz. Aus dem Jahr 1980 stammt eine doppelblinde, plazebokontrollierte Studie mit 215 Patienten, darunter 84 mit akuter Bronchitis (Germouty et al. 1980). Laut dieser Publikation soll es unter der oralen Gabe von Acetylcystein zu einer Zunahme des Sputumvolumens und der Expektoration, einer Verflüssigung des Sputums, einer Abnahme der Hustenintensität und einer Verbesserung der Peak-Flow-Messungen, auch bei den Patienten mit einer akuten Bronchitis, gekommen sein. Leider wurden die Ergebnisse nur als Summe der Gruppen akute Bronchitis, chronische Bronchitis und „komplizierte Infektionen“ dargestellt. Die Behauptung, die Gabe von Acetylcystein habe auch die akute Bronchitis positiv beeinflusst, wurde nicht durch konkrete Zahlenangaben gestützt. Zudem fehlten aussagekräftige Angaben zu den Patientenkollektiven. 
In zwei Anwendungsbeobachtungen aus den Jahren 1991 und 1992 wurde die orale Gabe von Acetylcystein bei chronischer und akuter Bronchitis als effektiv und gut verträglich beurteilt (Gerards und Vits 1991, Volkl und Schneider 1992). Für den Wirkstoff Ambroxol liegen keine kontrollierten Studien zur Anwendung bei der akuten Bronchitis vor. Für den Einsatz bei der chronischen Bronchitis wurden widersprüchliche Ergebnisse präsentiert (Gillissen et al. 2001).

Somit kann also festgestellt werden, dass für die Indikation der akuten Bronchitis keine ausreichende Therapieeffizienz von Mukolytika nachgewiesen werden konnte. Bezüglich der Diagnose des oberen Atemwegsinfektes sind keine kontrollierten Studien zur Effektivität der Gabe von Mukolytika bekannt. Dem steht ein Anteil von 91\% Verordnungen aus der Gruppe der Husten- und Erkältungspräparate bei dieser Diagnose gegenüber.

Als Prädiktoren für die Verordnung von Husten- und Erkältungspräparaten waren lediglich die Symptome Husten und Schnupfen signifikant. Auffällig war jedoch die insgesamt hohe Verordnungsrate bei allen untersuchten Symptomen bei $80 \%$ und mehr. Die Entscheidung zur Verabreichung wurde daher weitestgehend unabhängig von den Patientensymptomen getroffen. Die weitere Differenzierung des Symptoms Husten zeigt, dass sowohl trockener als auch feuchter, produktiver Husten zu einer signifikant höheren Verordnung führte. Weißlicher und gelblicher Auswurf waren jeweils signifikant mit der Gabe dieser Präparate assoziiert. Dabei war die Farbe des Auswurfs offensichtlich unerheblich.

Bei den erhobenen Untersuchungsergebnissen waren sowohl pathologische Atem- als auch Nebengeräusche ohne ausschlaggebenden Einfluss. Auffälliger Weise waren gerötete Tonsillen statistisch signifikant mit der Rezeptierung von Husten- und Erkältungspräparate verknüpft. Bei der weiteren Analyse dieses Ergebnisses ließ sich nachweisen, dass das Symptom gerötete Tonsillen zwar mit einer hohen Rate an derartigen Verordnungen assoziiert war, allerdings nur bei $14,4 \%$ der Patienten mit diesem Symptom die Diagnose einer akuten Tonsillitis gestellt wurde. Gerötete Tonsillen wurden von den beobachteten Ärzten offensichtlich nicht als Marker einer akuten Tonsillitis, sondern als Symptom eines oberen Atemwegsinfektes gewertet. 
Demgegenüber bestand bei dem Symptom Tonsillen mit Stippchen/ Belägen eine signifikant geringere Rezeptierung der hier zur Diskussion stehenden Präparate. Dies korrespondierte mit einer niedrigen Rate von Verordnungen bei der Diagnose der akuten Tonsillitis. Hier stand wohl der Gedanke einer gezielten antibakteriellen Therapie mittels eines Antibiotikums im Sinne einer kausalen Therapie im Vordergrund.

Höheres Alter sowie Berufstätigkeit waren ohne Einfluss auf das Verordnungsverhalten der Ärzte. Dies unterstützt zusammen mit dem bisher gezeigten weitgehenden Fehlen von Einflussfaktoren die These einer Verordnung dieser Präparate aus eher sekundären Überlegungen. Hummers-Pradier et al. (1999) verwiesen in diesem Zusammenhang auf die Möglichkeit der Befriedigung des (ärztlicherseits vermuteten) Patientenwunsches nach einer Verschreibung durch die Rezeptierung symptomatischer Medikamente. Weiterhin betonten sie, dass niedergelassene deutsche Ärzte zum Zeitpunkt der Datenerhebung nach Fallzahlen vergütet wurden und somit auch Patienten mit unkomplizierten akuten Krankheiten einen Einfluss auf das Einkommen hatten. Die Verschreibung von solchen Präparaten sollte die Patienten demnach möglicherweise an die Praxis binden.

Bei der Analyse arztspezifischer Faktoren fand sich lediglich bezüglich der Praxisgröße eine signifikante Differenz. Ärzte aus Praxen mit mehr als 1500 Scheinen verordneten signifikant mehr Präparate der Gruppe der Husten- und Erkältungspräparate. Hier könnte ein größerer Budgetspielraum eine Rolle spielen. Möglicherweise führte aber auch die große Patientenzahl zur routinemäßigen Gabe dieser Substanzen auf Grund von Zeitdruck während der Konsultation (Watkins et al. 2003).

Antitussiva stellten neben den Expektoranzien die zweitgrößte Gruppe innerhalb der Husten- und Erkältungspräparate dar. Insgesamt erhielten 59 Patienten (21,6\%) ein Antitussivum. Bei der Untersuchung von Kriterien zu deren Verordnung fällt auf, dass es dabei keinen signifikanten Unterschied zwischen Patienten mit unproduktiv trockenem Husten einerseits und produktivem Husten andererseits gab. Diese wurden offensichtlich nicht nur bei unproduktivem, quälendem und belastendem Husten angewendet, sondern auch entgegen der Pathophysiologie, bei sinnvollem produktivem Husten. Hier wurden mit hohem Nebenwirkungsspektrum behaftete 
Präparate (z.B. Suchtpotential der Opioide) ohne strikte Indikationstellung benutzt, deren klinischer Effekt zudem umstritten ist (Gillissen et al. 2001). So fanden Eccles et al. (1992) bei 91 Patienten mit einem oberen Atemwegsinfekt, dass Kodein keinen besseren antitussiven Effekt als die Plazebogabe hatte.

Andererseits ist es sinnvoll, dass die Patienten in der vorliegenden Studie signifikant häufiger ein Antitussivum erhielten, wenn sie über verstärkten Husten in der Nacht klagten. Allerdings wurde eine Anamnese bezüglich einer Zunahme des Hustens in der Nacht nur bei 22 der 146 Patienten mit Husten erhoben $(15,1 \%)$.

Wenn keine oder nur wenige unspezifische Patientencharakteristika zur Medikamentenverordnung führten und zudem die wissenschaftliche Evidenz der Therapie weitgehend fehlt, muss die Gabe dieser Präparate offensichtlich auf einer sekundären Ebene motiviert sein. Diese Zusammenhänge wurden bereits in Kapitel 1.6 dargestellt.

\subsection{Diagnostik und medikamentöse Therapie der 4 häufigsten Krankheitsbilder}

\subsubsection{Diagnostik und medikamentöse Therapie des oberen Atemwegsinfektes}

In der vorgestellten Untersuchung stand sowohl bei Erst- als auch bei Folgekontakten die Diagnose des oberen Atemwegsinfektes im Vordergrund. Es vergingen durchschnittlich 4,3 $( \pm$ 4,6) Tage bis zur Erstkonsultation des Arztes. Bezogen auf die 4 häufigsten Krankheitsbilder konsultierten Patienten mit einem oberen Atemwegsinfekt am schnellsten den Arzt. In einer Studie von Klein (2000) bei 317 deutschen Patienten mit neu aufgetretenen Symptomen einer akuten respiratorischen Erkrankung suchten 38\% der Betroffenen den Arzt innerhalb von 24 Stunden auf, innerhalb von 48 Stunden 61\%. Als mögliche Gründe dafür könnten, so Klein (2000), neben subjektivem Befinden und psychischer Konstitution auch der jeweilige Wochentag und die Situation am Arbeitsplatz und in der Familie sein.

Turner (1997) beschreibt die häufigsten Symptome des oberen Atemwegsinfektes: verstopfte und laufende Nase, Niesen, Halsschmerzen und Husten. Die Inkubationszeit beträgt 1 bis 2 Tage, den 
Höhepunkt der Symptomatik findet man nach 2 bis 4 Tagen. Gwaltney et al. (1994) nennen ebenfalls die verstopfte und laufende Nase als die charakteristischen Merkmale und Husten und Halsschmerzen als weitere oft auftretende Symptome.

In einer Studie zu einer Handlungsempfehlung bei Erkältungen beschreibt Barwitz (1999 b) anhand von retrospektiven Daten die Häufigkeit der typischen Krankheitssymptome eines banalen Erkältungsinfektes. Dabei waren Schnupfen (95\%), Halsschmerzen (70\%), Husten (80\%), Fieber (20\%) und Unwohlsein (60\%) die häufigsten Symptome.

In der vorliegenden Beobachtung wurde das Symptom Husten am häufigsten geschildert. Die multiple logistische Regressionsanalyse ergab die Symptome Schnupfen, Husten, Ohrenschmerzen und Heiserkeit, die mit der Diagnosestellung oberer Atemwegsinfekt assoziiert waren.

Bei oberen Atemwegsinfekten handelt es sich um eine zumeist selbst limitierende Erkrankung (Mainous III et al. 1996, English und Bauman 1997, Barwitz 1999 b). Auf die bislang beschränkten Möglichkeiten der evidenzbasierten Therapie oberer Atemwegsinfekte wurde bereits in Kapitel 1.4 eingegangen. Trotzdem erhielten 90\% der Erstkontakte ein Präparat aus der Gruppe der Husten- und Erkältungspräparate und 16\% ein Antibiotikum. Medikamentöse Verordnungsraten zwischen $45 \%$ und $65 \%$ wurden in vergleichbaren Studien nachgewiesen (English und Bauman 1997, Mainous III et al. 1996). Angaben über die Häufigkeit der Antibiotikaverordnungen bei Atemwegsinfekten sind in der Literatur breit gestreut. Sie variieren zwischen $11,5 \%$ in Deutschland (Hummers-Pradier et al. 1999), 20\% in den Niederlanden (Kuyvenhoven et al. 1993), 24\% in Japan (Kawamoto und Igarashi 1997) und bis zu 60\% in den USA (Mainous III et al. 1996, Gonzales et al. 1997).

\subsubsection{Diagnostik und medikamentöse Therapie der akuten Bronchitis}

Die Diagnose der akuten Bronchitis wurde am zweithäufigsten gestellt. In den Empfehlungen der Deutschen Atemwegsliga zur Prophylaxe und Therapie von bronchialen Infektionen (Worth et al. 1998) wird die akute Bronchitis als eine mit Husten und häufiger Exsudatbildung einhergehende 
Entzündung der Bronchialschleimhaut definiert. In der vorliegenden Arbeit klagten 100\% der Erstkontakte und 95,8\% der Folgekontakte über Husten, 78,3\% der ersten Gruppe und 83,3\% der Zweiten über Auswurf. Bei den Untersuchungen wurde nicht bei allen Patienten eine Auskultation der Lunge durchgeführt, obwohl eine Pneumonie eine potentielle Komplikation darstellt (Worth et al. 1998).

Die Ergebnisse der vorgenommenen logistischen Regressionsanalyse zeigen eine starke Assoziation zum Husten und zur Auskultation pathologischer Atem- bzw. Nebengeräusche. Ferner bestand eine Verknüpfung zu den Symptomen Abgeschlagenheit und Auswurf.

In einer Untersuchung von Verheij et al. (1995) zur Beeinflussung der Aktivitäten des täglichen Lebens bei 79 Patienten mit akuter Bronchitis wurden verstärkter Husten, eitriger Auswurf, Dyspnoe, Kopfschmerzen, Halsschmerzen, eine verstopfte Nase und Fieber als die häufigsten Symptome geschildert. Nach einer Woche klagte noch die Mehrheit der Patienten über die genannten Symptome, die meisten waren jedoch nach 2 Wochen verschwunden.

Gonzales et al. (1998) beschreiben in einer Studie zu den klinischen Merkmalen der akuten Bronchitis und deren Assoziation zur Verordnung von Antibiotika die folgenden dominierenden Symptome: Husten, Auswurf, laufende Nase, Halsschmerzen. Bei der körperlichen Untersuchung wurden der gerötete Rachen, geschwollene Halslymphknoten und Pfeifen und Giemen als pathologische Auskultationsgeräusche bei den Patienten als pathologische Befunde diagnostiziert. 55\% der Patienten waren zwischen 18 und 44 Jahren alt. 60\% suchten den Arzt innerhalb der 1 . Woche nach Krankheitsbeginn auf.

Die akute Bronchitis wird in der Mehrzahl der Fälle als eine durch Viren verursachte Entzündung gesehen (Worth et al. 1998); es kann nur selten von einer bakteriellen Genese ausgegangen werden. Die genaue Ätiologie bleibt jedoch auch oftmals im Unklaren. So konnten Jonsson et al. (1997) in einer Untersuchung an 140 Patienten mit den Symptomen einer akuten Bronchitis bei 84\% keinen Virus- oder Bakteriennachweis durch Anstiege der Antikörpertiter oder des CRP nachweisen. Dies steht in Einklang mit den Empfehlungen der Paul-Ehrlich-Gesellschaft für Chemotherapie e.V. und der Deutschen Atemwegsliga e.V. zur rationalen Therapie bakterieller 
Atemwegsinfektionen (Vogel et al. 2000). Demnach wird die Bronchitis bei sonst gesunden Erwachsenen als eine zumeist selbst limitierende Erkrankung bezeichnet, bei der eine antimikrobielle Therapie mit wenigen Ausnahmen nicht erforderlich ist. $\mathrm{Zu}$ diesen Ausnahmen, bei denen der Einsatz eines Antibiotikums erwogen werden sollte, zählen:

1. Patienten mit Grunderkrankungen (Diabetes mellitus, maligne Grunderkrankungen, Immuninkompetenz),

2. ältere Patienten

3. Patienten mit mehr als einer Woche andauernden Symptomen.

Vogel et al. (2000) halten aber eine antimikrobielle Therapie beim Auftreten von eitrigem Sputum als Hinweis für eine bakterielle Superinfektion für gerechtfertigt, was jedoch der bereits zitierten Studie von Winther et al. (1984) widerspricht (s. Kapitel 1.3). Eine weitere Schwäche dieser Empfehlungen ist die unklare Definition älterer Patienten und der eng gesteckte Zeitraum von einer Woche Symptomdauer als Indikation einer möglichen Antibiotikatherapie. Zudem wird diese nicht durch Literaturangaben auf der Basis der evidenzbasierten Medizin begründet.

In verschiedenen Metaanalysen wurde die Wirkung von Antibiotika bei akuter Bronchitis beurteilt. Es konnten nur marginale Einflüsse auf den Krankheitsverlauf festgestellt werden. So zeigen die Ergebnisse einer von Bent et al. (1999) durchgeführten Metaanalyse der zwischen den Jahren 1966 bis 1998 publizierten englischsprachigen Studien zur Antibiotikatherapie bei akuter Bronchitis keine signifikanten Unterschiede zwischen den Behandlungs- und Plazebogruppen hinsichtlich der Dauer des purulenten Sputums, des Hustens und der Arbeitsunfähigkeit. Untersucht wurden dabei Patienten mit einem normalen Röntgenbild des Thorax, bei denen keine Lungenvorerkrankungen vorlagen und die mit Erythromycin, Doxycyclin oder Trimethoprim/ Sulfamethoxazol behandelt wurden. Die Autoren halten eine antibiotische Therapie daher für unnötig, gerade auch im Hinblick auf eine mögliche Resistenzselektion.

Smucny et al. kommen in ihrem Cochrane Review (2003) nach der Metaanalyse von 9 Studien mit 750 Patienten mit einer akuten Bronchitis zu dem Ergebnis, dass es nur einen mäßigen Vorteil für die Anwendung von Antibiotika bei dieser Diagnose, vor allem im Hinblick auf mögliche Nebenwirkungen, die bei deren Anwendung signifikant erhöht waren, gibt. Patienten, 
die so behandelt wurden, hatten weniger Husten und er war von kürzerer Dauer. Der produktive Husten verringerte sich und die Patienten fühlten sich kürzer krank. Sie zeigten jedoch keine Verbesserung bei der Bewertung durch die untersuchenden Ärzte.

Arrol und Kenealy (2001) kritisieren allerdings die Ergebnisse der Metaanalysen zum Thema akute Bronchitis. Ihrer Meinung nach gibt es keine einheitliche Definition der akuten Bronchitis. Eine bakterielle Bronchitis könne nur durch die Röntgenaufnahme des Thorax von einer Pneumonie unterschieden werden. In einer Metaanalyse sollten nicht nur Symptome (Husten, produktives oder eitriges Sputum), sondern auch pathologische Untersuchungsbefunde bei der Auskultation (Giemen, Rasselgeräusche) als Zeichen eines tiefen Atemwegsinfektes berücksichtigt werden und der Benefit einer antibiotischen Behandlung dieser Patienten im Rahmen von Studien überprüft werden. Die Autoren kommen anhand der Bewertung von 7 Studien, die ihren Kriterien gerecht werden, zu dem Schluss, dass Patienten mit diesen Zeichen eines tiefen Atemwegsinfektes antibiotisch behandelt werden sollten und solche ohne diese Merkmale eher von der Gabe von Bronchodilatatoren profitieren würden.

Trotz der genannten Studiendaten werden bei diesem Krankheitsbild häufig Antibiotika verordnet, was in einer Vielzahl von Studien belegt wird. Die Häufigkeit der Antibiotikaverschreibungen schwankten zwischen $85 \%$ in den USA (Gonzales 1998), 68\% in den Niederlanden (Verheij et al. 1995) und 38,6\% in Deutschland (Hummers-Pradier et al. 1999). In der vorliegenden Untersuchung erhielten $80,4 \%$ der Patienten bei der Erstkonsultation ein Antibiotikum, wobei fast 50\% ein Makrolidantibiotikum verschrieben wurde.

Doch auch für die neueren Makrolidantibiotika konnten bislang keine überzeugenden Wirksamkeitsnachweise bei der akuten Bronchitis erbracht werden. So untersuchten Evans et al. (2002) das Makrolidantibiotikum Azithromycin bei akuter Bronchitis in einer randomisierten, doppelblinden Kontrollstudie im Vergleich zur Gabe von Vitamin C bei 220 Patienten. Beide Gruppen erhielten zusätzlich Dextromethrophan und einen Albuterolinhalator. Die Einnahme von Azithromycin ergab keine signifikanten Vorteile bezüglich der täglichen Aktivitäten und der Nebenwirkungen gegenüber Vitamin C. $81 \%$ der Patienten betrachteten die Anwendung des Albuterolinhaltors als subjektiv wirksam. 
In der vorliegenden Studie wurden am meisten Husten- und Erkältungspräparate verordnet, wobei über $80 \%$ der Patienten Expektoranzien anwenden sollten. Auf die fehlende Evidenz dieser Präparate wurde bereits in Kapitel 4.9 verwiesen. In anderen Ländern fanden sich wesentlich niedrigere Verordnungsraten der Expektoranzien. In den Niederlanden erhielten beispielsweise 25\% ein Mukolytikum (Verheij et al. 1995), in den USA spielt die Verordnung eines solchen Medikamentes keine Rolle (Gonzales et al. 1998).

Die Verschreibung von Antiasthmatika bei akuter Bronchitis stellte mit einem Anteil von 13,0\% an den Gesamtverordnungen bei Erstkontakten eher die Ausnahme dar. Die Wirksamkeit von Albuterol in Form eines Inhalates im Vergleich zu einem Plazeboinhalator wurde von Hueston (1994) bei 46 Patienten mit produktivem Husten untersucht, der nicht länger als 30 Tage bestand. Dessen Dauer wurde bei den Patienten signifikant gesenkt, die mit Albuterol behandelt wurden. Dieser Effekt blieb auch unter der Berücksichtigung des Zigarettenkonsums oder der gleichzeitigen Einnahme von Antibiotika bestehen.

\subsubsection{Diagnostik und medikamentöse Therapie der akuten Tonsillitis}

Die Diagnose der akuten Tonsillitis wurde in der vorliegenden Studie am dritthäufigsten gestellt. In Deutschland liegt ihre Auftreten bei 2 Millionen Fällen pro Jahr (Elies 2000). Der im Vordergrund stehende Beratungsanlass in diesem Fall sind akut aufgetretene Halsschmerzen. In unserer Studie klagten 95,2\% der Erstkontakte und 88,9\% der Folgekontakte über dieses Symptom. Der Arzt steht dabei vor dem Problem zwischen einer potentiell gefährlichen Streptokokkenangina und einer meist selbst limitierten Viruspharyngitis unterscheiden zu müssen (Meyer et al. 2002).

Die Häufigkeit von Infektionen mit betahämolysierenden Streptokokken wurde in verschiedenen Studien untersucht. Dabei konnten Streptokokkenprävalenzen zwischen $17 \%$ und $45 \%$ bei erwachsenen Patienten durch Abstriche und eine anschließende Kultur nachgewiesen werden (Centor et al. 1981, Dobbs 1996, Dagnelie et al. 1996, Howe et al. 1997, Meyer et al. 2002). Bei 
Kindern und Jugendlichen wurden mit 54\% z. T. noch höhere Prävalenzen festgestellt (Breese 1977).

Anhand von Punktebewertungen (sog. Scores) wurde von mehreren Autoren versucht, auf der Basis von anamnestischen, klinischen und laborchemischen Parametern, Entscheidungshilfen zu entwickeln um mit einer möglichst hohen Vorhersagewahrscheinlichkeit zwischen einer Streptokokkenangina und einer Viruspharyngitis unterscheiden zu können (Meyer et al. 2002). Breese (1977) entwickelte eine an 670 Jugendlichen validierte Entscheidungshilfe, bei der 9 Variablen nach gewichteten Punktwerten addiert werden mussten. Beim Überschreiten eines festgelegten Symptomscores wurde eine Wahrscheinlichkeit von $77,8 \%$ für eine Streptokokkenangina angegeben. Das von Centor et al. (1981) an 284 erwachsenen Patienten mit Halsschmerzen entwickelte Modell berücksichtigte 4 gleichgewichtige Variablen (Tonsillenbeläge, vergrößerte und druckschmerzhafte regionäre Lymphknoten, Fehlen von Husten und Fieber). Lagen alle 4 Kriterien vor, wurde eine Vorhersagewahrscheinlichkeit von 56 \% für eine Streptokokkeninfektion angegeben. Dobbs (1996) untersuchte Symptome und Befunde bei Personen mit Halsschmerzen mit dem Ziel zwischen denen mit Streptokokkennachweis und solchen ohne diesen Erreger unterscheiden zu können. Die durchgeführte multiple logistische Regressionsanalyse mit schrittweiser Elimination ergab eine unabhängige positive Korrelation zu einem Alter von weniger als 11 Jahren; Myalgien und Schmerzen oder stark vergrößerte Lymphknoten für eine Streptokokkeninfektion und eine negative Korrelation für das Vorliegen von Husten oder Ohrenschmerzen. Die Merkmale einzeln betrachtet erbrachten keine zuverlässige Unterscheidung; erst die Kombination aus mehreren anamnestischen Merkmalen und den Untersuchungsbefunden (B-Score-System) ergab eine Sensitivität und eine Spezifität von jeweils 71\%. McIsaac et al. (2000) werteten Daten von 621 Patienten mit Halsschmerzen aus, die in 97 kanadischen Hausarztpraxen erfasst wurden; dabei handelte es sich fast bei einem Drittel um Kinder im Alter zwischen 3 und 14 Jahren. Sie ermittelten eine Sensitivität von $85 \%$ und eine Spezifität von 92,1\% für das Vorhandensein eines Streptokokkeninfektes durch die Berechnung eines Punktwertes der sich aus der Addition von 5 gleich gewichteten Kriterien (Fieber $>38^{\circ} \mathrm{C}$, fehlender Husten, schmerzhafte Lymphknoten, geschwollene oder belegte Tonsillen, Alter zwischen 3 und 14 Jahren) zusammensetzte. 
Meyer et al. (2002) überprüften die dargestellten Entscheidungshilfen von Breese, Dobbs, McIsaac und Centor hinsichtlich ihrer Validität an 124 erwachsenen Halsschmerzpatienten in einer hausärztlichen Praxis. Sie fanden heraus, dass der von Centor (1981) entwickelte Score mit den Bewertungskriterien vergrößerte und/oder druckschmerzhafte regionäre Lymphknoten, Fieber, fehlender Husten, Tonsillenbeläge den anderen Scores überlegen war. Als ergänzende Kriterien konnten von den Autoren noch die Symptome Muskelschmerz und akuter Erkrankungsbeginn ermittelt werden.

Bei Betrachtung der in der vorliegenden Arbeit erhobenen anamnestischen Daten und Untersuchungsbefunde fällt auf, dass 42,9 \% der Erstkontakte und 22,2\% der Folgekontakte mit der Diagnose einer akuten Tonsillitis über Husten klagten, ein Kriterium, das bei Centor (1981) und Dobbs (1996) negativ mit einer Streptokokkeninfektion korrelierte und nach diesen Autoren einen Hinweis für eine virale Genese darstellt. Auch klagten nicht alle Patienten über Halsschmerzen. Von den insgesamt 30 Patienten mit der Diagnose einer akuten Tonsillitis bekamen zwar 23 ein Antibiotikum, aber nur ein Patient erfüllte die im Centor-Score genannten Kriterien (pathologischer Palpationsbefund der Halslymphknoten, Fieber, fehlender Husten und Tonsillenbeläge) einer Streptokokkeninfektion.

Eine weitere Möglichkeit der Diagnostik von Infektionen mit betahämolysierenden Streptokokken sind der Streptokokkenschnelltest und der Rachenabstrich mit Kultur. Für den Schnelltest liegen Daten über eine Sensitivität zwischen 65\% und 85\% vor; die Spezifität wird mit einem Wert von über 90\% angegeben (Gabler-Sandberger 1998, Dagnelie et al. 1996). Die Sensitivität der Schnelltests konnte in der Kombination mit typischen, häufig durch einen Streptokokkeninfekt bedingten Befunden (Fieber $>38,0^{\circ} \mathrm{C}$, fehlender Husten, Exsudat auf den Tonsillen, vordere Lymphknotenschwellung) gesteigert werden.

Barwitz (1999 a) empfiehlt in seiner vorgestellten Leitlinie auf der Basis der evidenzbasierten Medizin zum Beratungsanlass Halsschmerzen einen Rachenabstrich für einen Streptokokkenschnelltest und/oder eine Kultur nur in Zweifelsfällen vorzunehmen. Bei rezidivierenden Streptokokkeninfekten sollte die Diagnose mit einem solchen Abstrich, kultureller Austestung, Antibiogramm und Antistreptolysintiter-Bestimmung gesichert werden. 
Nach Meinung von Meyer et al. (2002) und Donner-Banzhoff (1999) sollte der Streptokokkenschnelltest erst dann angewandt werden, wenn zuvor durch eine entsprechende Anamnese gemäß der beschriebenen Scores eine diagnostische Eingrenzung stattgefunden hat um die Prävalenz zu erhöhen.

In einer Übersichtsarbeit von Touw-Otten und Johansen (1992) werden die Diagnostik und Therapie der akuten Tonsillitis in 17 europäischen Staaten beschrieben. Bakteriologische Tests zur Diagnostik, so die Autoren, wurden in Deutschland, Polen, Österreich und den Niederlanden sehr selten benutzt; ein hoher Prozentsatz von 70-96\% an bakteriologischen Tests fand man in der Türkei, Rumänien, Israel, Jugoslawien, Finnland und Griechenland. In den meisten Staaten wurden bei über 90\% der Patienten ein Antibiotikum verordnet, wobei in den Niederlanden mit $68 \%$ die wenigsten Verordnungen und in Rumänien, Frankreich und Belgien mit nahezu 100\% die meisten Verschreibungen registriert wurden. In Westdeutschland erhielten $87 \%$ der Patienten ein Antibiotikum. In den meisten Ländern wurde Penicillin verschrieben (68\%). Dabei wurde in den nördlichen Ländern wie Finnland, Schweden sowie den Niederlanden und Westdeutschland eher orales Penicillin verabreicht, während in den südlichen Ländern oft auch parenterales Penicillin eingesetzt wurde.

Gabler-Sandberger (1998) wertete 4,8 Millionen Antibiotikaverordnungen zur Therapie der akuten Tonsillitis in Deutschland aus dem Jahr 1997 aus. Den Spitzenwert mit 54,3\% der Verordnungen erzielte Penicillin, gefolgt von Makroliden (17,5\%), Aminopenicillinen (19,9\%), Cephalosporinen (10,9\%), Tetracyclinen (3,3\%) und Cotrimazol (3,0\%). In der vorliegenden Studie wurde Penicillin am häufigsten verordnet (52,4\%), gefolgt von Makroliden (23,8\%).

Del Mar et al. (2003) resümierten in einer Metaanalyse 25 Studien zum Thema Antibiotika bei Halsschmerzen aus dem Zeitraum 1945 bis 1999. Die genannten Substanzen senkten die Inzidenz des rheumatischen Fiebers, der akuten Otitis media und der akuten Sinusitis. Außerdem wurden die Symptome Kopfschmerzen, Halsschmerzen und Fieber gelindert. Die Krankheitsdauer verkürzte sich um durchschnittlich 16 Stunden bei der Gabe von Antibiotika. Die Effekte waren nach Ansicht der Autoren jedoch nur gering ausgeprägt, sodass ihr Einsatz kritisch beurteilt werden sollte. 
Am ehesten profitieren von einer Antibiotikatherapie Patienten mit nachgewiesenem Streptokokkeninfekt. So konnten Dagnelie et al. (1996) in einer randomisierten, plazebokontrollierten Doppelblindstudie zur Wirksamkeit von Penicillin V bei 239 Patienten in 37 niederländischen Praxen mit dem Verdacht auf eine Streptokokkeninfektion der Tonsillen zeigen, dass nur diejenigen von dieser Art der Behandlung profitierten, bei denen tatsächlich eine solche Infektion nachgewiesen wurde. Dieser Effekt bezog sich auf die Besserung der Halsschmerzen und die Senkung des Fiebers sowie auf eine 1 bis 2-tägige schnellere Genesung. Die Autoren fordern vor dem Hintergrund dieser Ergebnisse die Durchführung eines Streptokokkenschnelltestes.

Little et al. (1997) untersuchten 3 Behandlungsstrategien bei 716 Patienten mit den klinischen Symptomen einer Pharyngitis oder Tonsillitis. Die 1. Gruppe erhielt ein Antibiotikum über 10 Tage, die 2. Gruppe keins und die 3. Gruppe ein Rezept für ein Antibiotikum mit der Anweisung es einzunehmen, wenn sich die Symptome nach 3 Tagen nicht gebessert hätten. Dabei zeigte sich, dass 69\% der Patienten der letzten Gruppe ihre Verschreibung nicht nutzten. Es gab keine signifikanten Unterschiede der Besserung der Symptome am 3. Tag, der Krankheitsdauer, der Fehlzeit in der Schule sowie bei der Arbeit oder der Patientenzufriedenheit. Die mittlere Fieberdauer betrug jedoch in der 1. Gruppe einen Tag im Vergleich zu 2 Tagen in der 2. und 3. Gruppe $(\mathrm{p}=0,04)$. Die Meinungen zur Wirksamkeit des Antibiotikums gingen jedoch deutlich auseinander. Patienten der 1. Gruppe hielten Antibiotika für wirkungsvoll und verlangten bei ähnlichen Beschwerden häufiger wieder eine derartige Verordnung im Vergleich zu den Patienten der Gruppen 2 und 3. Die Autoren kommen zu dem Schluss, dass die Zufriedenheit der Patienten mit der Behandlung einen starken Einfluss auf die Genesung hat und psychosoziale Faktoren (Krankheitslegitimation bei der Arbeit und zu Hause) beachtet werden sollten.

Thomas et al. (2000) beurteilten in einer Metaanalyse von 22 Studien alternative Behandlungsmethoden zur nicht-antibiotischen Therapie von Halsschmerzen. Eine Symptomlinderung innerhalb von 24 Stunden konnte durch Paracetamol und nicht-steroidale Antiphlogistika erzielt werden, wobei Ibuprofen die größte Effektivität zugeschrieben wurde. Die Wirksamkeit von Paracetamol und nicht-steroidalen Antiphlogistika wurde auch für 
Krankheitszeichen beschrieben, die länger als 24 Stunden andauerten. Ferner wurde die Effektivität von Präventivmaßnahmen diskutiert. Auch eine bessere Patientenaufklärung und Kommunikation mit ihm könnten die Symptome rascher lindern. Die Autoren kritisieren jedoch, dass es bisher keine Studien gibt, die den Erfolg alternativer Therapien im Vergleich zur Antibiotikatherapie untersuchen.

\subsubsection{Diagnostik und medikamentöse Therapie der akuten Sinusitis}

Die Diagnose der akuten Sinusitis stellt den Allgemeinarzt vor ein schwieriges diagnostisches Problem. Seine Möglichkeiten beschränken sich meistens auf die Anamnese und die körperliche Untersuchung. In der vorliegenden Studie wurde bei allen Patienten mit dieser Diagnose eine Palpation der Nasennebenhöhlen und bei 90\% eine Inspektion des Mund-Rachenraumes durchgeführt. Bei 4 (12,1\%) wurden zusätzliche Laboruntersuchungen angeordnet. 4 Patienten (12,1\%) wurden überwiesen, davon jeweils 2 zum Facharzt für Hals-Nasen-Ohrenheilkunde und 2 zum Radiologen zum Röntgen der Nasennebenhöhlen.

Auf der Grundlage epidemiologischer Daten schätzen Snow et al. (2001), dass nur 0,2\%-2\% aller viral bedingten oberen Atemwegsinfekte durch eine bakterielle Sinusitis kompliziert werden. Im Hinblick auf eine mögliche Verordnung von Antibiotika erscheint es besonders wichtig Kriterien zu finden, die eine akute bakterielle Sinusitis mit einer möglichst hohen Sensitivität und Spezifität erkennen lassen und andere Diagnosen wie z.B. die viral-katarrhalischen Sinusitis, die allergischen Rhinitis, den oberen Atemwegsinfekt oder Erkrankungen der Zähne ausschließen (Snow et al. 2001).

Ein einfaches Unterscheidungskriterium zwischen viral und bakteriell bedingter akuter Sinusitis stellen Snow et al. (2001) in ihren evidenzbasierten Leitlinien zur Therapie der akuten Sinusitis anhand der Symptomdauer dar. Symptome, die für eine akute bakterielle Sinusitis sprechen sind eitriges Nasensekret, Schmerzen oder Druckschmerzhaftigkeit im Bereich des Oberkiefers und des Gesichtes (vornehmlich einseitig) sowie Zahnschmerzen. Dauern die Symptome weniger als 7 Tage, so die Autoren, sei eine bakterielle Sinusitis unwahrscheinlich. In der hier präsentierten 
Studie war die Symptomdauer bei 39,4\% der Patienten mit der Diagnose einer akuten Sinusitis länger als 7 Tage; 46,2\% dieser Patienten erhielten ein Antibiotikum.

Zur Differenzierung zwischen einem oberen Atemwegsinfekt und einer akuten Sinusitis führten Hueston et al. (1998) eine retrospektive Studie mit 734 Patienten durch. Die an einer akuten Sinusitis leidenen Patienten waren häufiger älter, weiblich, Raucher, hatten eine längere Symptomdauer und eine allergische Rhinitis in der Vorgeschichte. Am stärksten waren die 4 Faktoren druckschmerzhafte Nasennebenhöhlen, Druckgefühl in den Nasennebenhöhlen, postnasales Sekret und eine Sinusitis in der Vorgeschichte sowie verfärbtes Sekret, Übelkeit, Kopfschmerzen und die Krankheitsdauer als weitere Faktoren mit der Diagnose einer akuten Sinusitis assoziiert.

Williams et al. (1992) beschreiben folgende 5 unabhängige prädiktive Faktoren dieser Erkrankung, die sie in einer prospektiven Studie zum Vergleich zwischen Anamnese und klinischer Untersuchung einerseits und Befunden einer durchgeführten NativRöntgenuntersuchung andererseits erhoben haben: Zahnschmerzen im Oberkiefer, pathologischer Diaphanoskopiebefund, geringe Besserung der Symptomatik bei der Anwendung von abschwellenden Mitteln und Antihistaminika, vom Patienten geschildertes verfärbtes Nasensekret, mukös-eitriges Sekret bei der Untersuchung. Die klinische Einschätzung des untersuchenden Arztes als Summe der Befunde war jedoch genauer als jedes Symptom einzeln betrachtet.

Van Buchem et al. (1995) untersuchten die Zusammenhänge zwischen dem klinischen Bild der Sinusitis und objektivierbaren Befunden, die mittels Rhinoskopie, Röntgen der Nasennebenhöhlen, Ultraschall und Punktion der Nasennebenhöhlen bei 113 Patienten mit der Verdachtsdiagnose einer akuten Sinusitis maxillaris erhoben wurden. Die Diagnose wurde häufiger bei Frauen (67\%) als bei Männern (33\%) gestellt. 76\% der Patienten waren zwischen 18 und 38 Jahren alt. Anders als in den zuvor genannten Studien fanden die Autoren heraus, dass bei dem Verdacht auf eine akute Sinusitis maxillaris die Anamnese und der klinischen Untersuchungsbefund zur Diagnosestellung wenig hilfreich waren, da sie keine ausreichende Korrelation zur apparativen Diagnostik aufwiesen. 
In der vorliegenden Untersuchung konnten mittels multipler logistischer Regressionsanalyse folgende Faktoren ermittelt werden, die die Hausärzte zur Diagnose einer akuten Sinusitis veranlassten: pathologischer Palpationsbefund der Nasennebenhöhlen, schmerzende Nasennebenhöhlen und Kopfschmerzen. Dies steht in Einklang mit den beschriebenen Ergebnissen von Hueston et al. (1998).

Antibiotika bei akuter Sinusitis wurden nach 2 in den USA durchgeführten Studien 98\% der Patienten verschrieben (Hueston et al. 1998, Dosh et al. 2000), wobei bei es sich bei Hueston et al. (1998) am häufigsten um Sulfamethoxazol/Trimethoprim handelte. In Deutschland erhielten 33\% ein Antibiotikum (Hummers-Pradier et al. 1999)

In der vorliegenden Erhebung sollten 57,9\% der an akuter Sinusitis Erkrankten nach dem Erstkontakt ein Antibiotikum einnehmen, nach der Folgekonsultation 42,9\%. Dabei wurde Doxycyclin als Monosubstanz oder in Kombination mit Expektoranzien am häufigsten verschrieben. In den vorliegenden Metaanalysen (De Ferranti et al. 1998, Williams et al. 2003) konnte eine Reduktion der Symptomdauer gezeigt werden. De Ferranti et al. (1998) untersuchten weiterhin die Wirksamkeit verschiedener Antibiotika basierend auf 27 Studien und 2717 Patienten. Danach boten andere Antibiotika (Cephalosporine, Makrolide) keine Vorteile im Vergleich zu Amoxicillin. Auch Williams et al. (2003) konnten keine Unterschiede in der Wirksamkeit verschiedener Antibiotikagruppen nachweisen.

Zur Wirksamkeit von Doxycyclin bei akuter Sinusitis, dem in der vorliegenden Studie klar favorisierten Antibiotikum, legten Stalman et al. (1997) eine plazebokontrollierte randomisierte Doppelblindstudie vor. Sie zeigten, dass Doxycyclin zusätzlich zur Behandlung mit abschwellenden Nasentropfen, Inhalation und ggf. der Einnahme von Paracetamol nicht effektiver war als die alleinige Basisbehandlung.

Wie weit sich Expertenmeinungen von diesen evidenzbasierten Aussagen entfernen können, belegen die Empfehlungen einer Expertengruppe der Paul-Ehrlich-Gesellschaft für Chemotherapie e.V. und der Deutschen Atemwegsliga e.V. zur rationalen Therapie bakterieller 
Atemwegsinfektionen (Vogel et al. 2000), die eine antibiotische Therapie für erforderlich halten.

Sie empfehlen die Anwendung von oralen Cephalosporinen mit Staphylokokkenwirksamkeit, Aminopenicillinen gegebenenfalls mit Beta-Lactamase-Inhibitoren, Makroliden oder Fluorchinolonen der Gruppe 3.

Am häufigsten wurden in der vorliegenden Studie jedoch Medikamente aus der Gruppe der Husten- und Erkältungspräparate verordnet (94,7\% der Erstkontakte 57,1\% der Folgekontakte). Bei Hummers-Pradier et al. (1999) wurden diese Mittel mit 82,2\% etwas weniger verschrieben. Die kritische Beurteilung dieser Medikamente erfolgte bereits in Kapitel 4.9.

Der Anteil der Rhinologikaverordnung fiel mit 26,3\% bei Erstkontakten und 21,4\% bei Folgekontakten niedrig aus. Eine Metaanalyse von Taverner et al. (2003) hatte eine, zumindest für den kurzzeitigen Einsatz, relevante Wirksamkeit dieser Präparate nachgewiesen.

\subsection{Abhängigkeit der Diagnosestellung von geschilderten Symptomen und erhobenen Untersuchungsbefunden}

Wie bereits in Kapitel 1.4 dargestellt gehen die Autoren Glasziou und Del Mar (2002) sowie Hueston et al. (2000 a) davon aus, dass es sich bei den 4 am häufigsten diagnostizierten Krankheitsbildern um Varianten der gleichen klinischen Erkrankung mit unterschiedlichen anatomischen Manifestationen handelt. Die Ergebnisse der multiplen logistischen Regressionsanalyse zeigen, dass die jeweilige Diagnosestellung durch die teilnehmenden Hausärzte mit bestimmten Symptomen und Untersuchungsbefunden positiv bzw. negativ verknüpft war. Ein oberer Atemwegsinfekt wurde beim Vorliegen der Symptome Schnupfen, Husten, Ohrenschmerzen und Heiserkeit diagnostiziert, während pathologische Untersuchungsbefunde der Lunge damit negativ assoziiert waren. Die Diagnose der akuten Bronchitis wurde im Gegensatz dazu in den meisten Fällen auf Grund pathologischer Lungenuntersuchungsbefunde und den Symptomen Husten und Abgeschlagenheit gestellt. Bei der akuten Tonsillitis bestand eine Assoziation zu dem Symptom Halsschmerzen und pathologischer Untersuchungsbefunde des Rachens und der Halslymphknoten; die Symptome Husten und Schnupfen waren negativ mit dieser Diagnose assoziiert. An pathologische 
Untersuchungsbefunde bei der Palpation der Nasennebenhöhlen und die Symptome der schmerzenden Nasennebenhöhlen und Kopfschmerzen war die Diagnose der akuten Sinusitis geknüpft, während es eine negative Assoziation zum Symptom Husten gab.

Diese Ergebnisse der multivariaten Analyse zeigen, dass die beobachteten Ärzte auf Grund ihrer erhobenen Anamnese und den Befunden zwischen den einzelnen Diagnosen unterschieden. Dies drückt sich auch darin aus, dass bei etwa 10\% der Patienten mehr als eine Diagnose gestellt wurde. Die Ärzte versuchten offenbar Symptome und Untersuchungsbefunde zu kategorisieren. Der z.T. deutliche Unterschied bei der Rate der Antibiotikaverordnungen (16\% beim oberen Atemwegsinfekt und $80 \%$ bei der akuten Bronchitis) unterstützt die Annahme, dass die teilnehmenden Ärzte durchaus zwischen verschiedenen Krankheitsbildern differenzierten und nicht von einer einheitlichen Erkrankung mit lediglich unterschiedlicher Ausprägung ausgingen.

\subsection{Schlussfolgerungen}

Folgende Schlussfolgerungen für die Therapie von Atemwegsinfekten können, abgeleitet von den hier präsentierten Daten und der zitierten Literatur, getroffen werden:

1. Stärkere Gewichtung der Selbstmedikation und der nichtmedikamentösen Therapie,

2. Empfehlung zum Selbstkauf frei verkäuflicher Medikamente wie Paracetamol, Nasentropfen; keine Rezeptierung,

3. Weitergabe von sogenannten „Infozepten“ an die Patienten (z.B. KVN-Rezept-Info Nr.4) als Alternative zur Rezeptierung von Medikamenten,

4. keine Verordnung von Arzneimitteln ohne wissenschaftlichen Beleg ihrer Wirksamkeit für diese Indikation (z.B. Acetylcystein, Ambroxol),

5. Verzicht auf den Einsatz von Antibiotika bei unkomplizierten oberen Atemwegsinfekten,

6. Antibiotikagabe bei akuter Tonsillitis nur bei der Wahrscheinlichkeit einer Streptokokkeninfektion (klinische Scores, Streptokokkenschnelltest),

7. Verordnung von Antibiotika bei akuter Bronchitis und akuter Sinusitis nur in Ausnahmefällen bei sogenannten Risikopatienten (multimorbide Patienten, Patienten mit Immunsuppression), 
8. keine generelle Wiedereinbestellung von Patienten.

Diese Schlussfolgerungen könnten als Diskussionsgrundlage für die Entwicklung bzw. Weiterentwicklung einer nationalen Leitlinie zur Diagnostik und Therapie von Atemwegsinfekten dienen. Die Beachtung der genannten Punkte fordert jedoch vom Arzt die Bereitschaft Zeit für eine ausreichende Aufklärung des Patienten über sein Krankheitsbild zu investieren. Nur so kann ein gutes Arzt-Patienten-Verhältnis geschaffen werden und der Patient verlässt höchstwahrscheinlich zufrieden die Praxis, auch wenn er kein Rezept erhält (Himmel et al. 1997, von Ferber 2000, Lundkvist et al. 2002). Dieser Ansatz steht jedoch im Gegensatz zum gegenwärtigen Vergütungssystem, das eine gesprächsorientierte, beratende Medizin leider wenig honoriert. 


\section{Zusammenfassung}

Akute Atemwegsinfekte gehören $\mathrm{zu}$ den häufigsten Krankheitsbildern in der Allgemeinarztpraxis. Ziel der Erhebung war es Daten zur Diagnostik und Therapie von Atemwegsinfekten in der hausärztlichen Praxis zu analysieren. Während einer jeweils eintägigen Hospitation bei 30 Fachärzten für Allgemeinmedizin wurde deren Vorgehensweise bei allen Patienten mit einem akuten Atemwegsinfekt dokumentiert, die im Beobachtungszeitraum den Arzt konsultierten. Insgesamt wurden 273 Patienten mit akuten Atemwegsinfekten erfasst (21,4\% $( \pm 6,4)$ der Gesamtkonsultationen im Erhebungszeitraum), die in Erst- und Folgekontakte unterschieden wurden.

Die häufigsten von Patienten geschilderten Symptome waren Husten, Schnupfen und Halsschmerzen. Nahezu alle wurden körperlich untersucht; dabei standen die Auskultation der Lunge und die Inspektion des Nasen- und Rachenraumes im Vordergrund. Die Diagnosen oberer Atemwegsinfekt, akute Bronchitis, akute Tonsillitis und akute Sinusitis wurden am häufigsten gestellt. Akute Exazerbationen chronisch obstruktiver Lungenerkrankungen spielten eine eher untergeordnete Rolle. Durchschnittlich erhielten Erstkonsultierende 2,1 $( \pm 1,0)$ Medikamente, Patienten mit Folgekontakt 1,3 $( \pm 1,1)$ Medikamente. Am häufigsten wurden Medikamente aus der Gruppe der Husten- und Erkältungspräparate verordnet (87,1\% der Erstkontakte und 52,9\% der Folgekontakte). 43,5\% der Erstkontakte und 29,9\% der Folgekontakte erhielten ein Antibiotikum. Die meisten Verordnungen entfielen auf die chemische Substanz Acetylcystein aus der Gruppe der Expektoranzien, gefolgt von Ambroxol und Paracetamol.

Die Symptome Husten, Schnupfen, Kopfschmerzen und gerötete Tonsillen sowie ein pathologischer Rachenbefund waren statistisch signifikant mit der Verordnung von Husten- und Erkältungspräparaten verknüpft. Ärzte aus Praxen mit mehr als 1500 Scheinen stellten deutlich mehr Rezepte für Husten- und Erkältungspräparate aus als kleinere Praxen.

Bei den Antibiotikaverordnungen standen Makrolide mit 37,5\% an 1. Stelle, gefolgt von Penicillinen (21,5\%) und Doxycyclin als Monosubstanz oder in Kombination mit Expektoranzien (20,8\%). Die Zahl der Verordnungen variierte stark in Abhängigkeit zur gestellten Diagnose. So 
erhielten 15,6\% der Patienten mit einem oberen Atemwegsinfekt, 57,6\% mit einer akuten Sinusitis, 80,4\% mit einer akuten Bronchitis und 85,7\% mit einer akuten Tonsillitis ein Antibiotikum. Signifikant häufiger mit einer Antibiotikaverordnung assoziiert waren die Symptome gelblich-grüner Auswurf, Abgeschlagenheit und Fieber. Bei den Untersuchungsbefunden zeigte sich eine Verbindung $\mathrm{zu}$ pathologischen Atem- und Nebengeräuschen bei der Auskultation, dem Vorhandensein von Stippchen oder Belägen auf den Tonsillen, einem pathologischen Palpationsbefund der Halslymphknoten, einem pathologischen Otoskopiebefund sowie einem pathologischen Palpationsbefund der Nasennebenhöhlen. Weiterhin verschrieben Ärzte, die älter als 50 Jahre waren und solche aus Gemeinschaftspraxen, signifikant häufiger Antibiotika.

17,9\% der Patienten machten Angaben zur Selbstmedikation, 12,1\% zur nichtmedikamentösen Therapie und der Verwendung von sogenannten „Hausmitteln". Nur wenige baten den Arzt um die Verschreibung eines bestimmten Präparates (2,9\%). Eine Empfehlung zum Selbstkauf von Medikamenten erfolgte eher selten (2,6\%). 34,8 \% der Patienten erhielten Empfehlungen zur nichtmedikamentösen Therapie, wobei die Empfehlung einer erhöhten Flüssigkeitszufuhr und Inhalationen im Vordergrund standen.

Die erhobenen Daten lassen vermuten, dass sich bei einer höheren Gewichtung der sogenannten „Hausmittel" und der Selbstmedikation ein deutliches Einsparpotential böte. Angesichts der hohen Zahl der Antibiotikaverordnungen bei Atemwegsinfekten sollte die entsprechende Indikationsstellung zur Antibiotikatherapie kritisch überdacht werden. Medikamente, deren Wirksamkeit für diese Indikation nicht wissenschaftlich belegt ist (z.B. Acetylcystein, Ambroxol), sollten nicht verordnet werden. 


\section{Anhang}

\section{DOKUMENTATIONSBOGEN ATEMWEGSINFEKTE}

Kassenpatient $\square$ ja $\square$ nein Geburtsjahr...... Geschlecht $\square$ männlich $\square$ weiblich

Beruf:

$\square$ Erstkontakt $\quad \square$ Folgekontakt

initial verordnete Therapie/Medikamente:

$\square$ Medikamente $\quad \square$ Dauermedikation:

$\square$ Selbstmedikation:

$\square$ Eigentherapie:

Raucher

$\square$ nein $\square$ ja

Zigaretten pro Tag:........

BESCHWERDEN seit ........Tagen

$\square$ Abgeschlagenheit $\square$ nein $\square$ ja

$\square$ Gliederschmerzen $\square$ nein $\square$ ja

$\square$ Kopfschmerzen $\quad \square$ nein $\square$ ja

$\square$ Schnupfen $\quad \square$ nein $\square$ ja

$\square$ Heiserkeit $\quad \square$ nein $\square$ ja

$\square$ Halsschmerzen $\quad \square$ nein $\square$ ja

$\square$ Ohrenschmerzen $\quad \square$ nein $\square$ ja

$\square$ Augenbeschwerden $\quad \square$ nein $\square$ ja

$\square$ Schmerzen NNH $\quad \square$ nein $\square$ ja

$\square$ Zahnschmerzen $\quad \square$ nein $\square$ ja

$\square$ Fieber $\quad \square$ nein $\square$ ja

$\square$ Husten $\quad \square$ nein $\square$ ja

$\square$ morgens verstärkt $\quad \square$ unproduktiv, trocken $\quad \square$ produktiv, feucht

Auswurf(Sputum) $\quad \square$ weißlich $\quad \square$ gelblich-grün $\quad \square$ blutig

$\square$ sonstige Beschwerden:

\section{UNTERSUCHUNG UND BEFUND}

körperliche Untersuchung $\square$ nein $\square$ ja

$\square$ Palpation / Perkussion Nasennebenhöhlen:

$\square$ Palpation der Halslymphknoten

$\square$ Inspektion der Mundhöhle und des Rachens:

Rachenhinterwand:

Tonsillen:

$\square$ Otoskopie:

$\square$ Perkussion der Lunge:

$\square$ Auskultation der Lunge:

$\square$ Stimmfremitus:

$\square$ Sonstiges:

$\square$ weitere diagnostische Untersuchungen: $\square$ nein $\quad \square$ ja durchgeführte Untersuchungen:

$\square$ Überweisung: $\quad \square$ nein $\quad \square$ ja

$\square$ Grund der Überweisung:

$\square$ Krankenhauseinweisung 


\section{THERAPIE}

\section{Empfohlene Medikamente}

$\square$ Kassenrezept

$\square$ Privatrezept.

$\square$ als Muster mitgegeben

$\square$ Selbstkauf empfohlen.

Arzneiwunsch des Patienten:

nein

nein

Bettruhe

Rauchverbot

Zusätzliche Empfehlun

$\square$ erhöhte Flüssigkeitszufuhr

$\square$ Inhalationen zu Hause

zu Hause

$\square$ Bestrahlung. ja.......

$\square$ ja

$\square$ Wadenwickel

$\square$ in der Praxis Sonstiges:....

$\square$ Arbeitsunfähigkeitsbescheinigung: $\square$ nein $\square$ ja $\square$ Dauer:

Wiedervorstellung erbeten: $\quad \square$ nein $\square$ wenn Beschwerden nicht abklingen

\section{DIAGNOSE (nach ICPC)}

$\square$ R72 Streptokokkenangina / Scharlach

$\square$ R74 Erkältungsschnupfen / oberer Atemwegsinfekt

$\square$ R75 akute / chronische Sinusitis

$\square$ R76 akute Tonsillitis

$\square$ R77 akute Laryngitis / Tracheitis

$\square$ R78 akute Bronchitis / Bronchiolitis

$\square$ R81 Pneumonie

$\square$ R83 andere Infektionen des respiratorischen Systems

$\square$ H71 akute Otitis media / Entzündung des Trommelfells

$\square$ H73 Tubenkatarrh

$\square$ R84 maligne Tumoren Trachea / Bronchien / Lunge

$\square$ R85 Andere maligne Tumoren

$\square$ R90 Hypertrophie / chronische Infektion der Tonsillen / Rachenmandeln

$\square$ R91 chronische Bronchitis / Bronchiektasen

$\square$ R95 Lungenemphysem / chronisch obstruktive Lungenerkrankungen

$\square$ R96 Asthma bronchiale

$\square$ R97 Heuschnupfen / allergische Rhinitis

$\square$ R99 andere Erkrankungen des respiratorischen Systems

$\square$ H70 Otitis externa

$\square$ H74 chronische Otitis media / andere Infektionen des Ohres 


\section{ANGABEN ZUR PRAXIS}

Anzahl der praktizierenden Ärzte:

$\square$ Praxisgemeinschaft $\square$ Gemeinschaftspraxis

Geschlecht $\square$ männlich $\square$ weiblich

Alter:

Dauer der Niederlassung:

Zusatzbezeichnungen:

Scheinzahl pro Quartal: $\square>500$

$\square$ 500-1000
$\square 1000-1500$
$\square 1500-2000$
$\square>2000$

\section{HOSPITATION}

Datum:

Gesamtzahl der am Tag der Hospitation behandelten Patienten:

Anzahl der akuten Atemwegsinfekte: 


\section{Literaturverzeichnis}

Adam D, Ferlinz R, Frei L, Knothe H, Lode H, Shah P (1991): Antibakterielle Therapie bei infektiösen Bronchialerkrankungen. Empfehlungen der Deutschen Liga zur Bekämpfung von Atemwegserkrankungen. MMW Munch Med Wochenschr 133, 308-312

Arroll B, Kenealy T (2001): Antibiotics for acute bronchitis. BMJ $\underline{322}$, 939-940

Arroll B, Kenealy T: Antibiotics for the common cold (Cochrane Review). Aus: The Cochrane Library, Ausgabe 1, Oxford 2003 (Update Software)

Arzneimittelkommission der deutschen Ärzteschaft (2000): Handlungsleitlinie - akute Atemwegsinfektionen. Z Arztl Fortbildung Qualitatssich $\underline{94}$, 133-136

Avorn J, Solomon DH (2000): Cultural and economic factors that (mis)shape antibiotic use: the nonpharmacologic basis for therapeutics. Ann Intern Med 133, 128-135

Barrett BP, Brown RL, Maberry R, Bobula JA, D`Alessio D (2002): Treatment of the common cold with unrefined echinacea. Ann Intern Med 137, 939-946

Barwitz HJK (1999 a): Beratungsanlass Halsschmerzen - was nützt eine Handlungsleitlinie? MMW Fortschr Med 141, 750-754

Barwitz HJK (1999 b): Erkältung: Rationelles Vorgehen in der Allgemeinarztpraxis. Z Allgemeinmed 75, 928-931

Bent S, Saint S, Vittinghoff E, Grady D (1999): Antibiotics in acute Bronchitis: a meta-analysis. Am J Med 107, 62-67

Bisno AL (1991): Group A streptococcal infections and acute rheumatic fever. N Engl J Med $\underline{325}, 783-793$

Bogdewic SP: Participant Observation; in: Doing Qualtitative Research; hrsg.v. Crabtree BJ, Miller WL; Sage Publications, Newbury Park 1992, 45-69

Bormann C, Däubener W, Abholz HH, Altiner A (2003): Bakterielle Erreger und Resistenzen bei 232 Patienten mit akutem produktivem Husten. Z Allgemeinmed 79, 193-199

Bradley CP (1992 a): Uncomfortable prescribing descisions: a critical incident study. BMJ $\underline{304}$, 294-296

Bradley CP (1992 b): Factors which influence the decision wether or not to prescribe: the dilemma facing general practitioners. BMJ $\underline{304}, 454-458$

Bradley CP, Riaz A, Tobias RS, Kenkre JE, Dassu DY (1998): Patient attitudes to over-thecounter drugs and possible professional responses to self-medication. Fam Pract $\underline{15}, 44-50$ 
Brede HD (1997): Atemwegserkrankungen. Ther Erfolg 1, 132-134

Breese BB (1977): A simple scorecard for the tentative diagnosis of streptococcal pharyngitis. Am J Dis Child 131, 514-517

Britten N (1995): Patients' demands for prescriptions in primary care. BMJ $\underline{310}, 1084-1085$

Britten N, Stevenson FA, Barry CA, Barber N, Bradley CP (2000): Misunderstandings in prescribing decisions in general practice: qualitative study. BMJ $\underline{321}, 1160-1161$

Bundesverband der Betriebskrankenkassen (2002): BKK-Statistik über die Arbeitsunfähigkeit 2000. http://www.bkk.de/ihre_bkk/statistika/download/an_2000_bkkstatistik.pdf, zugegriffen am 12.7.2002

Buser K, Kaul-Hecker U: Medizinische Psychologie, medizinische Soziologie. 3. Auflage; Gustav Fischer Verlag, Stuttgart 1991

Butler CC, Rollnick S, Pill R, Maggs-Rapport F, Stott N (1998): Understanding the culture of prescribing: qualitative study of general practitioners' and patients' perceptions of antibiotics for sore throats. BMJ $\underline{317}, 637-642$

Centor RM, Witherspoon JM, Dalton HP, Brody CE, Link K (1981): The diagnosis of strep throat in adults in the emergency room. Med Decis Making $\underline{3}$, 239-246

Cockburn J, Pit S (1997): Prescribing behaviour in clinical practice: patients' expectations and doctors' perceptions of patients' expectations - a questionnaire study. BMJ $\underline{315}, 520-523$

Couchman GR, Rascoe TG, Forjuoh SN (2000): Back-up antibiotic prescription for common respiratory symptoms. J Fam Pract $\underline{49}$, 907-913

Cox CM, Jones M (2001): Is it possible to decrease antibiotic prescribing in primary care? An analysis of outcomes in the management of patients with sore throat. Fam Pract 18, 9-13

Dagnelie CF, van der Graaf Y, de Melker RA (1996): Do patients with sore throat benefit from penicillin? A randomized double-blind placebo-controlled clinical trial with penicillin $\mathrm{V}$ in general practice. Br J Gen Pract $\underline{46}, 589-593$

D`Agostino RB, Weintraub M, Russel HK (1998): The effectiveness of antihistamines in reducing the severity of runny nose and sneezing: a meta-analysis. Clin Pharmacol Ther $\underline{64}, 579-$ 596

Dahmer J: Anamnese und Befund. 6. Auflage; Georg Thieme Verlag, Stuttgart 1988

Davey P, Rutherford D, Graham B, Lynch B, Maleck M (1994): Repeat consultations after antibiotic prescribing for respiratory infection: a study in one general practice. $\mathrm{Br} \mathrm{J}$ Gen Pract 44 , $509-513$ 
De Ferranti SD, Ioannidis JPA, Lau J, Anninger WV, Barza M (1998): Are amoxycillin and folate inhibitors as effective as other antibiotics for acute sinusitis? A meta-analysis. BMJ $\underline{317}$, 632-637

Del Mar CB, Glasziou PP, Spinks AB: Antibiotics for sore throat (Cochrane Review). Aus: The Cochrane Library, Ausgabe 1, Oxford 2003 (Update Software)

De Melker RA, Kuyvenhoven MM (1994): Management of upper tract infections in dutch family practice. J Fam Pract $\underline{38}, 353-357$

Deutsches Institut für medizinische Dokumentation und Information (DIMDI) im Auftrage des Bundesministeriums für Gesundheit: ICD-10-Diagnosenthesaurus, Version 3.0; Deutscher Ärzteverlag, Köln 1999

Dobbs F (1996): A scoring system for predicting group A streptococcal throat infection. Br J Gen Pract $\underline{46}, 461-464$

Donner-Banzhoff N (1999): Wie stellt der Allgemeinarzt seine Diagnose? Z Allgemeinmed $\underline{75}$, 744-749

Dosh SA, Hickner JM, Mainous AG, Ebell MH (2000): Predictors of antibiotic prescribing for nonspecific upper respiratory infections, acute bronchitis, and acute sinusitis. J Fam Pract $\underline{49}$, 407-414

Douglas RM, Chalker EB, Treacy B: Vitamin C for preventing the common cold (Cochrane Review). Aus: The Cochrane Library, Ausgabe 2, Oxford 2003 (Update Software)

Eccles R, Morris S, Jawad M (1992): Lack of effect of codeine in the treatment of cough associated with upper respiratory tract infection. J Clin Pharm Ther $\underline{17}, 175-180$

Elies W (2000): Therapie von Atemwegsinfektionen in der Praxis. Warum differenziert werden muss. Hausarzt $\underline{14}, 34-37$

English J A, Bauman KA (1997): Evidence-based management of upper respiratory infection in a family practice teaching clinic. Fam Med 29, 38-41

Evans AT, Husain S, Duraiaj L, Sadowski LS, Charles-Damte M, Wang Y (2002): Azithromycin for acute bronchitis: a randomised, double-blind, controlled trial. Lancet $\underline{359}$, 1648-1654

Evertz W, Haag H, Hahn K, Nadjafi-Kouzehkonani M, Kresse W (1999): Qualitätsförderung mit dem Praxiscomputer. Z Allgemeinmed 75, 939-941

Fahey T, Stocks N, Thomas T (1998): Systematic review of the treatment of upper respiratory tract infection. Arch Dis Child 79, 225-230

Flintrop J (2002): Die Selbstmedikation boomt. Dtsch Arztebl 99, 881 
Flückinger U (2001): Welches ist die optimale Behandlungsdauer der Gruppe-AStreptokokkenpharyngitis? HNO $\underline{49}, 253-255$

Gabler-Sandberger E (1998): Tonsillopharyngitis: Je nach Alter deutliche Unterschiede. Dtsch Arztebl 95, 1919-1920

Gerards HH, Vits U (1991): Therapie der Bronchitis. Fortschr Med 109, 707-710

Germouty J, Brocard H, Charpin J (1980): Kooperative Doppelblindstudie mit oralem Acetylcystein und Plazebo. Therapiewoche 30, 1984-1990

Gillissen A, Tasci S, Ewig S, Schäfer H, Zielen S (2001): Sinn und Unsinn von Antitussiva. Internist $\underline{42}, 134-142$

Glasziou P, Del Mar CB in: Clinical Evidence. 8. Ausgabe; hrsg. v. Barton S; BMJ Publishing Group, London 2002, 1583-1591

Gonzales R, Steiner JF, Sande MA (1997): Antibiotics prescribing for adults with colds, upper respiratory tract infections and bronchitis by ambulatory care physicians. JAMA $\underline{278}, 901-904$

Gonzales R, Barrett PH, Crane LA, Steiner JF (1998): Factors associated with antibiotic use for acute bronchitis. J Gen Intern Med 13, 541-548

Gonzales R, Barrett PH, Steiner JF (1999): The relation between purulent manifestations and antibiotic treatment of upper respiratory tract infections. J Gen Intern Med 14, 151-156

Gwaltney JM, Phillips CD, Miller RD, Riker DK (1994): Computed tomographic study of the common cold. N Engl J Med 330, 25-30

Hamm H: Allgemeinmedizin. 4. Auflage; Georg Thieme Verlag, Stuttgart 1988

Hamm RM, Hicks RJ, Bemben DA (1996): Antibiotics and respiratory infection: are patients more satisfied when expectations are met? J Fam Pract $\underline{43}$, 56- 62

Heikkinen T, Järvinen A (2003): The common cold. Lancet $\underline{361}, 51-59$

Hendley JO, Abbott RD, Beasley PP, Gwaltney JM (1994): Effect of inhalation of hot humidified air on experimental rhinovirus infection. JAMA $\underline{271}, 1112-1113$

Himmel W (2000): Selbstmedikation - ökonomische, sozialpharmakologische und toxikologische Aspekte. Dtsch Med Wochenschr 125, 401-407

Himmel W, Sandholzer H, Kochen MM (1995): Sickness certification in general practice. Eur J Gen Pract $\underline{1}, 161-166$ 
Himmel W, Lippert-Urbanke E, Kochen MM (1997): Are patients more satisfied when they receive a prescription? The effect of patient expectations in general practice. Scand J Prim Health Care $\underline{15}, 118-122$

Hirt M, Nobel S, Barron E (2000): Zinc nasal gel for the treatment of common cold symptoms: a double-blind, placebo-controlled trial. Ear Nose Throat J $\underline{79}, 778-782$

Hjortdahl P, Landaas S, Urdal P, Steinbakk M, Fuglerud P, Nygaard B (1991): C-reactive protein: a new rapid assay for managing infectious disease in primary health care. Scand $\mathrm{J}$ Prim Health Care $\underline{9}, 3-10$

Howe RW, Millar MR, Coast J, Whitfield M, Peters TJ, Brookes S (1997): A randomized controlled trial of antibiotics on symptom resolution in patients presenting to general practitioner with sore throat. Br J Gen Pract 47, 280-284

Howie JGR: Research in general practice. 2. Ausgabe; Chapman and Hall, London 1989

Hueston WJ (1994): Albuterol delivered by metered-dose inhaler to treat acute bronchitis. J Fam Pract $\underline{39}, 437-440$

Hueston WJ, Eberlein C, Johnson D, Mainous III AG (1998): Criteria used by clinicians to differentiate sinusitis from viral upper respiratory tract infection. J Fam Pract $\underline{46}, 487-492$

Hueston WJ, Mainous III AG, Dacus EN, Hopper JE (2000 a): Does acute bronchitis really exist? J Fam Pract $\underline{49}, 401-406$

Hueston WJ, Jenkins R, Mainous III AG (2000 b): Does drug treatment of patients with acute bronchitis reduce additional care seeking? Arch Fam Med 9, 997-1001

Hummers-Pradier E, Pelz J, Himmel W, Kochen MM (1999): Treatment of respiratory tract infections - a study in 18 general practices in Germany. Eur J Gen Pract $\underline{5}, 15-20$

Jackson JL, Lesho E, Peterson C (2000): Zinc and the common cold: a meta-analysis revisited. Nutrition $\underline{130}, 1512$ S-1515S

Jefferson TO, Tyrrell D: Antivirals for the common cold (Cochrane Review). Aus: The Cochrane Library, Ausgabe 1, Oxford 2003 (Update Software)

Jonsson JS, Sigurdsson JA, Kristinsson KG, Gudnadóttir M, Magnusson S (1997): Acute bronchitis in adults. How close do we come to its etiology in general practice? Scand J Prim Health Care $\underline{15}, 156-160$

Kawamoto R, Igarashi M (1997): Antibiotics for URIs in Japan. J Fam Pract $\underline{45}, 461$

Kirkpatrick GL (1996): The common cold. Prim Care 23, 657-675 
Klein R (2000): Wann kommt der Patient zum Arzt und was unternimmt er vorher? Allgemeinarzt 22, 1394-1398

Kochen MM (1988): Verordnungsverhalten niedergelassener Ärzte. Dtsch Med Wochenschr $\underline{113}$, 696-701

Kochen MM: Allgemeinmedizin. 1. Auflage; Hippokrates Verlag GmbH, Stuttgart 1992

Kochen MM (1994): Allgemeinärztliche Besonderheiten der Pharmakotherapie. Z Arztl Fortbild $\underline{88}, 647-654$

Kochen MM (2000): „The best prescription may be no prescription.“. Dtsch Med Wochenschr $\underline{125}, 401$

Korppi M, Kröger L (1992): C-reactive protein in viral and bacterial respiratory infection in children. Scand J Infect Dis $\underline{25}$, 207-213

Kravitz RL, Cope DW, Bhrany V, Leake B (1994): Internal medicine patients' expectations for care during office visits. J Gen Intern Med $\underline{9}, 75-81$

Kresken M, Haffner D, Studiengruppe der Paul-Ehrlich-Gesellschaft (2000): Resistenzsituation bei klinisch wichtigen Infektionserregern gegenüber Chemotherapeutika in Mitteleuropa. Chemother J $\underline{9}, 51-86$

Kuyvenhoven M, de Melker R, van der Velden K (1993): Prescription of antibiotics and prescibers' charakteristics. A study into prescription of antibiotics in upper respiratory tract infections in general practice. Fam Pract $\underline{10}, 366-370$

Lamberts H, Wood M: The International Classification of Primary Care (ICPC). Oxford University Press, Oxford 1987

Lamberts H: Episode-oriented epidemiology in family practice: the practical use of the International Classification of Primary Care (ICPC) as illustrated in patients with headache; in: Primary care research: traditional and innovative approaches; hrsg. v. Norton PG; Sage Publications, Inc., Newbury Park 1991, 40-71

Lamberts H, Wood M, Hofmans-Okkes E: The International Classification of Primary Care in the European Community. Oxford University Press, Oxford 1993

Lemmer B: Antitussiva und Expektoranzien; in: Arzneiverordnungsreport 1998; hrsg. v. Schwabe U, Paffrath D; Springer-Verlag, Berlin 1999, 173-193

Lemmer B: Antitussiva und Expektoranzien; in: Arzneiverordnungsreport 2001; hrsg. v. Schwabe U, Paffrath D; Springer-Verlag, Berlin 2002, 279-303

Lichte T (2001): KVN-Rezept-Info Nr. 4: Tipps für eine wirtschaftliche Arzneimitteltherapie bei Erkältungskrankheiten und grippalen Infekten. Niedersachs Arztebl 12, 67-68 
Lichte T, Lichte C, Fischer GC (1999): Das Infozept - die optimierte schriftliche Kommunikation in der Arztpraxis. Z Allgemeinmed 75, 563-567

Lichte T (2001): KVN-Rezept-Info Nr. 4: Tipps für eine wirtschaftliche Arzneimitteltherapie bei Erkältungskrankheiten und grippalen Infekten. Niedersachs Arztebl 12, 67-68

Little P, Williamson I, Warner G, Gould C, Gantley M, Kinmonth AL (1997): Open randomised trial of prescribing strategies in managing sore throat. BMJ $\underline{314}, 722-727$

Lundkvist V, Åkerlind I, Borgquist L, Mölstad S (2002): The more time spend on listening, the less time spent on prescribing antibiotics in general practice. Fam Pract $\underline{19}$, 638-640

Macfarlane J, Holmes W, Gard P, Macfarlane R, Rose D, Weston V, Leinonen M, Saikku P, Myint S (2001): Prospective study of the incidence, etiology and outcome of adult lower respiratory tract illness in the community. Thorax $\underline{56}, 109-114$

Macknin ML, Mathew S, van der Brug-Medendorp S (1990): Effect of inhaling heated vapor on symptoms of the common cold. JAMA $\underline{264}, 989-991$

McIsaac WJ, Goel V, To T, Low DE (2000): The validity of a sore throat score in family practice.

CMAJ $\underline{16}, 811-815$

Mähler L (1996): Grippe und Erkältung. Teil 1: Ätiologie von Grippe und Erkältung, Physiologie der Nase und Immunologie. Allgemeinarzt 17, 1873-1885

Mainous III AG, Hueston WJ, Clark JR (1996): Antibiotics and upper respiratory infection. Do some folks think there is a cure for the common cold? J Fam Pract $\underline{42}$, 357-361

Mainous III AG, Zoorob RJ, Oler MJ, Haynes DM (1997): Patients knowledge of upper respiratory infections. Implications for antibiotic expectations and unnecessary utilisation. J Fam Pract $\underline{45}, 75-83$

Marshall I: Zinc in the treatment of the common cold (Cochrane Review). Aus: The Cochrane Library, Ausgabe 1, Oxford 2003 (Update Software)

Melbye H, Aasebo U, Straume B (1991): Symptomatic effect of inhaled fenoterol in acute bronchitis: a placebo-controlled double-blind study. Fam Pract $\underline{8}, 216-222$

Melchart D, Linde K, Fischer P, Kaesmayr J: Echinacea for preventing and treating the common cold (Cochrane Review). Aus: The Cochrane Library, Ausgabe 1, Oxford 2003 (Update Software)

Meltzer EO, Charous BL, Busse WW, Zinreich SJ, Lorber RR, Danzig MR (2000): Added relief in the treatment of acute recurrent sinusitis with adjunctive mometasone furoate nasal spray. $\mathrm{J}$ Allergy Clin Immunol 106, 630-637 
Meyer F, Beck C, Baum E, Donner-Banzhoff N (2002): Die Diagnose der Streptokokkentonsillitis. Kritische Prüfung diagnostischer Entscheidungsregeln. Z Allgemeinmed $\underline{78}, 248-253$

Monto AS, Sullivan KM (1993): Respiratory illness in the community. Epidemiol Infect 110, $145-160$

Morgenstern C: Der Schnupfen; in: Rowohlt Lesebuch „Gute Besserung"; hrsg. v. Blänsdorf A; Rowohlt Taschenbuch Verlag, Reinbeck 2001

Murray S, Del Mar CB, O`Rourke P (2000). Predictors of antibiotic prescription by GPs for respiratory tract infections: a pilot. Fam Pract $\underline{17}, 386-388$

Olivier C (2000): Rheumatic fever - is it still a problem? J Antimicrob Chemother $\underline{45}, 13-21$

Palmer K (1990): Prescribing. Practitioner 234, 888-890

Pillau H, Abholz HH: Der Notfall in der Allgemeinmedizin; in: Allgemeinmedizin, 1. Auflage; hrsg. v. Kochen MM; Hippokrates Verlag GmbH, Stuttgart 1992, 128-132

Poole PJ, Black PN: Mucolytic agents for chronic bronchitis (Cochrane Review). Aus: The Cochrane Library, Ausgabe 2, Oxford 2003 (Update Software)

Rote Liste $^{\circledR}$ Service GmbH (Hrsg): Rote Liste 1997. Editio Cantor Verlag, Aulendorf 1996

Sanchez-Menegay C, Hudes ES, Cummings SR (1992): Patient expectations and satisfaction. J Gen Intern Med 7, 432-434

Schmidt, S, Engelhardt S, Ziesché R, Gesenhues ST: Praxisleitfaden Allgemeinmedizin. 1. Auflage; Gustav Fischer Verlag, Stuttgart 1996

Schmitz W: Antibiotika und Chemotherapeutika; in: Arzneiverordnungsreport 1998; hrsg. v. Schwabe U, Paffrath D; Springer-Verlag, Berlin 1999, 64-81

Schnur S, Exner H (1997): Akute respiratorische Erkrankungen in der Hausarztpraxis. Z Allgemeinmed $\underline{73}, 477-479$

Schroeder K, Fahey T: Over-the-counter medications for acute cough in children and adults in ambulatory settings (Cochrane Review). Aus: The Cochrane Library, Ausgabe 2, Oxford 2003 (Update Software)

Schwabe U: ATC-Code: Anatomomisch-therapeutisch-chemische Klassifikation für den deutschen Arzneimittelmarkt. 1. Auflage; GKV-Arzneimittelindex, Bonn 1995

Schwabe U: Überblick über die Arzneiverordnungen im Jahre 1997; in: Arzneiverordnungsreport 1998; hrsg. v. Schwabe U, Paffrath D; Springer-Verlag, Berlin 1999 a, 1-19 
Schwabe U: Bewertung von Arzneimitteln; in: Arzneiverordnungsreport 1998; hrsg. v. Schwabe U, Paffrath D; Springer-Verlag, Berlin 1999 b, 579-615

Schwabe U: Überblick über die Arzneiverordnungen im Jahre 2001; in: Arzneiverordnungsreport 2001; hrsg. v. Schwabe U, Paffrath D; Springer-Verlag, Berlin 2002 a, 1-18

Schwabe U: Antibiotika und Chemotherapeutika; in: Arzneiverordnungsreport 2001; hrsg. v. Schwabe U, Paffrath D; Springer-Verlag, Berlin 2002 b, 131-155

Schwabe U: Einsparpotentiale; in: Arzneiverordnungsreport 2001; hrsg. v. Schwabe U, Paffrath D; Springer-Verlag, Berlin 2002 c, 785-831

Seppäla H, Klaukka T, Vuopio-Varkila J, Muotiala A, Helenius H, Lager K, Huovinen P (1997): The effect of changes in the consumption of macrolide antibiotics on erythromycin resistance in group A streptococci in Finland. N Engl J Med 337, 441-6

Shim C, King M, Williams MH (1987): Lack of effect of hydration on sputum production in chronic bronchitis. Chest $\underline{\text { 92, 679-682 }}$

Singh M: Heated, humidified air for the common cold (Cochrane Review). Aus: The Cochrane Library, Ausgabe 1, Oxford 2003 (Update Software)

Snow V, Mottur-Pilson C, Hickner JM (2001): Principles of appropriate antibiotic use for acute sinusitis in adults. Ann Intern Med 134, 495-497

Smucny J, Fahey T, Becker L, Glazier R: Antibiotics for acute bronchitis (Cochrane Review). Aus: The Cochrane Library, Ausgabe 1, Oxford 2003 (Update Software)

Stalman W, van Essen GA, van der Graaf Y, de Melker RA (1997): The end of antibiotic treatment in adults with acute sinusitis-like complaints in general practise? A placebo-controlled double-blind randomized doxycycline trial. Br J Gen Pract 47, 794-799

Stalman WA, van Essen GA, van der Graaf Y (2001): Determinants for the course of acute sinusitis in adult general practice patients. Postgrad Med J $\underline{77}, 778-782$

Stockley RA, O'Brien C, Pye A, Hill SL (2000): Relationship of the sputum colour to nature and outpatient management of acute exacerbations of COPD. Chest $\underline{117}, 1638-1645$

Tauchnitz C (2000): Strategien des Einsatzes von oralen Antibiotika in der Praxis. Z Allgemeinmed $\underline{76}, 554-559$

Taverner D, Bickford L, Draper M: Nasal decongestants for the common cold (Cochrane Review). Aus: The Cochrane Library, Ausgabe 1, Oxford 2003 (Update Software)

Thomas M, Del Mar CB, Glasziou P (2000): How effective are treatments other than antibiotics for acute sore throat? Br J Gen Pract $\underline{50,}$ 817-820 
Thust W, Lieschke L (1999): Ergebnisse der Ärztestatistik zum 31. Dezember 1998. Dtsch Arztebl 96, Suppl 3-15

Touw-Otten FWMM, Johansen KS (1992): Diagnosis, antibiotic treatment and outcome of acute tonsillitis: report of a WHO regional office for Europe study in 17 European countries. Fam Pract $\underline{9}, 255-262$

Turner RB (1997): Epidemiology, pathogenesis, and treatment of common cold. Ann Allergy Asthma Immunol 78, 531-540

Van Buchem L, Peeters M, Beaumont J, Knottnerus JA (1995): Acute maxillary sinusitis in general practice: the relation between clinical picture and objective findings. Eur J Gen Pract $\underline{1}$, $155-160$

Verheij T, Hermans J, Kaptein A, Mulder J (1995): Acute bronchitis: course of symptoms and restrictions in patients`daily activities. Scand J Prim Health Care 13, 8-12

Vogel F, Worth H, Adam D, Elies W, Ewig S, Höffken G, Lode H, Lorenz J, Scholz H, Stille W (2000): Rationelle Therapie bakterieller Atemwegsinfektionen. Chemother J 9, 3-23

Volk1 KP, Schneider B (1992): Therapy of respiratory tract diseases with N-Acetylcysteine. Fortschr Med 110, 346-350

Volmer T, Gehlert D, Nowak D, Fabel H, Konietzko N (2000): Kosten der bedeutendsten Atemwegs- und Lungenkrankheiten in Deutschland. Pneumonologie 54, S29

von Ferber L (2000): Patienten erwarten nicht immer ein Rezept. Dtsch Arztebl 97, 1350-1351

Walluf-Blume D (1997): Neues Bewusstsein für die eigene Gesundheit. Pharm Ztg $\underline{41}$, 3-5

Watkins C, Harvey I, Carthy P, Moore L, Robinson E, Brawn R (2003): Attitudes and behaviour of general practitioners and their prescribing costs: a national cross sectional survey. Qual Saf Health Care 12, 29-34

Webb S, Lloyd M (1994): Prescribing and referral in general practice: a study of patients' expectations and doctors' actions. Br J Gen Pract 44, 165-169

Williams JW, Simel DL, Roberts L, Samsa GP (1992): Clinical evaluation for sinusitis. Making the diagnosis by history and physical examination. Ann Intern Med 117, 705-710

Williams JW, Aguilar C, Cornell J, Chiquette E, Dolor RJ, Makela M, Holleman DR, Simel DL: Antibiotics for acute maxillary sinusitis (Cochrane Review). Aus: The Cochrane Library, Ausgabe 2, Oxford 2003 (Update Software)

Wilson AA, Crane LA, Barrett PH, Gonzales R (1999): Public beliefs and the use of antibiotics for acute respiratory illness. J Gen Intern Med 14, 658-662 
Winther B, Brofeldt S, Gronborg H, Mygind N, Pedersen M, Vejlsgaard R (1984): Study of bacteria in the nasal cavity and nasopharynx naturally acquired common colds. Acta Otolaryngol $\underline{98}, 315-320$

Worth H, Adam D, Handrick W, Leupold W, Lode H, Loos U, Marre R, Mauch H, Schaberg T, Shah P (1998): Prophylaxe und Therapie von bronchialen Infektionen. Pneumonologie 52, 232237 


\section{Danksagung}

Danken möchte ich Herrn Prof. Dr. med. Michael M. Kochen für die Überlassung des Dissertationsthemas und Frau Dr. Hummers-Pradier für die Betreuung. Mein weiterer Dank gilt den teilnehmenden Ärzten für die Ermöglichung der Hospitation und die freundliche Aufnahme in ihrer Praxis. 


\section{Lebenslauf}

Am 22. Juni 1966 wurde ich als Tochter des Diplom-Kaufmanns Dr. rer. pol. Karl-Josef Hecker und seiner Ehefrau Anne-Marie Hecker, geb. Schmücker in Brilon geboren. Von 1972-1976 besuchte ich die Grundschule Alfeld und anschließend das Gymnasium Alfeld, wo ich 1985 das Abitur ablegte.

Nach einem halbjährigen Praktikum im Altenheim der Inneren Mission in Alfeld begann ich eine Ausbildung zur Krankenschwester im St. Bernward-Krankenhaus Hildesheim, die ich 1989 erfolgreich beendete. Von 1989 bis 1993 arbeitete ich als OP-Schwester im St. BernwardKrankenhaus Hildesheim und absolvierte in dieser Zeit eine berufsbegleitende Weiterbildung zur OP-Fachschwester. Im Oktober 1993 begann ich das Medizinstudium an der MHH Hannover. 1995 bestand ich die ärztliche Vorprüfung, ein Jahr später den ersten Abschnitt der ärztlichen Prüfung. Nach erfolgreich bestandenem 2. Staatsexamen wechselte ich 1998 an die GeorgAugust-Universiät Göttingen und absolvierte mein Praktisches Jahr im Albert-SchweitzerKrankenhaus Northeim. Von 2000 bis 2002 arbeitete ich als Ärztin im Praktikum in der Klinik für Allgemein- und Viszeralchirurgie im Albert-Schweitzer-Krankenhaus Northeim und erhielt am 21. Mai 2002 die Vollapprobation als Ärztin.

Seit 1998 bin ich mit Dr. med. Thomas Fischer verheiratetet und befinde mich nach der Geburt unserer Söhne Jonas (2000) und Benedikt (2002) in der Elternzeit. 